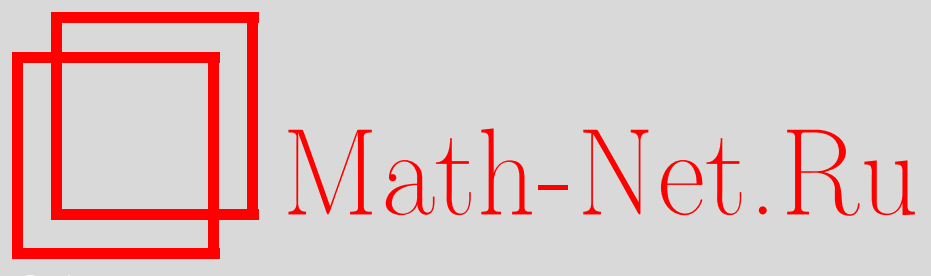

А. Гогатишвили, В. Д. Степанов, Редукционные теоремы для весовых интегральных неравенств на конусе монотонных функций, УМН, 2013, том 68, выпуск 4, 3-68

DOI: https://doi.org/10.4213/rm9538

Использование Общероссийского математического портала Math-Net.Ru подразумевает, что вы прочитали и согласны с пользовательским соглашением http://www . mathnet.ru/rus/agreement

Параметры загрузки:

IP: 54.147 .182 .235

26 апреля 2023 г., 16:19:54

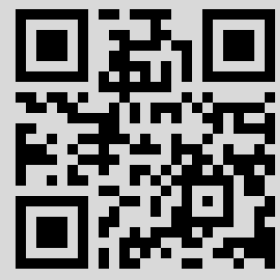




\section{Редукционные теоремы для весовых интегральных неравенств на конусе монотонных функций}

\section{А. Гогатишвили, В. Д. Степанов}

В работе дается обзор результатов, связанных с редукцией интегральных неравенств с положительными операторами в весовых пространствах Лебега на вещественной полуоси на конусе монотонных функций к некоторым неравенствам на конусе неотрицательных функций, для доказательства которых имеется больше возможностей. При этом случай монотонных операторов является новым. В качестве приложения для ряда операторов Вольтерра получена полная характеризация при всех возможных параметрах суммирования.

Библиография: 118 названий.

Ключевые слова: весовое пространство Лебега, конус монотонных функций, весовое интегральное неравенство, принцип двойственности, ограниченные операторы, редукционная теорема.

DOI: $10.4213 / \mathrm{rm} 9538$

\section{СОДЕРЖАНИЕ}

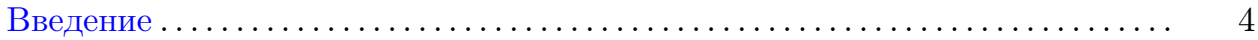

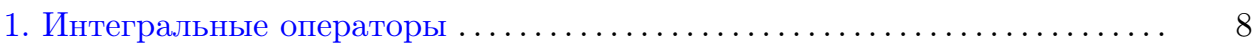

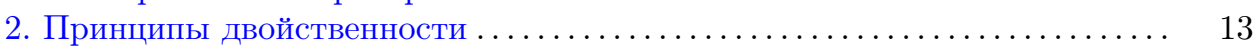

2.1. Принцип двойственности Сойера ..................... 14

2.2. Неравенство Харди на монотонных функциях .............. 20

3. Редукционные теоремы ............................... 25

3.1. Редукционные теоремы для монотонных операторов при $0<q \leqslant$

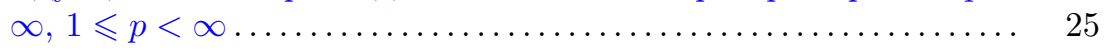

3.2. Редукционные теоремы для квазилинейных операторов при $0<$

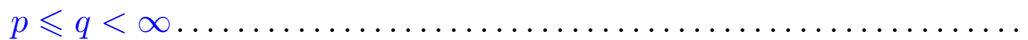

3.3. Редукционные теоремы для операторов Вольтерра при $0<q<$

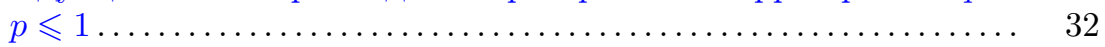

3.4. Дальнейшие результаты и комментарии ................. 53

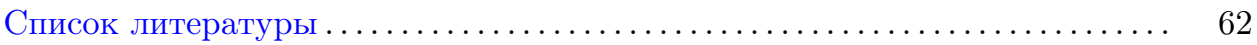

Работа первого автора выполнена при поддержке Научного Агентства Республики Чехия (гранты № 201-08-0383 и P201-13-14743S) и Института математики Чешской Академии наук (грант RVO: 67985840). Работа второго автора выполнена при поддержке РФФИ (гранты № 12-01-00524 и 12-01-00554) и Дальневосточного отделения Российской академии наук (гранты № 12-I-OMN-01 и 12-II-C0-01M-002).

(C) А. ГОГАтишВили, В. Д. СТЕПАНов, 2013 


\section{Введение}

В работе дается обзор по весовым интегральным неравенствам в пространствах Лебега на конусах монотонных функций на полуоси.

Пусть $\mathbb{R}_{+}:=[0, \infty)$. Обозначим $\mathfrak{M}$ пространство всех измеримых по Лебегу функций на $\mathbb{R}_{+}$. При $0<p \leqslant \infty$ положим

$$
\|f\|_{p}= \begin{cases}\left(\int_{\mathbb{R}_{+}}|f(x)|^{p} d x\right)^{1 / p}, & 0<p<\infty, \\ \underset{x \in \mathbb{R}_{+}}{\operatorname{esssup}}|f(x)|, & p=\infty,\end{cases}
$$

и определим пространство Лебега $L_{p}$ как совокупность всех $f \in \mathfrak{M}$ таких, что $\|f\|_{p}<\infty$.

Известной задачей функционального анализа является нахождение необходимых и/или достаточных условий выполнения при фиксированных параметpax $0<p, q \leqslant \infty$ интегральных неравенств вида

$$
\left(\int_{\mathbb{R}_{+}}\left|w(x) \int_{\mathbb{R}_{+}} k(x, y) f(y) u(y) d y\right|^{q} d x\right)^{1 / q} \leqslant C\left(\int_{\mathbb{R}_{+}}|f(x)|^{p} d x\right)^{1 / p}, \quad f \in \mathfrak{M}
$$

где $k(x, y)$ - измеримое по совокупности переменных ядро, $u(x)$ и $w(x)$ - локально суммируемые весовые функции, а константа $C \geqslant 0$ не зависит от $f$ и выбирается наименьшей из возможных, т. е. равной норме $\|\mathscr{K}\|_{L_{p} \rightarrow L_{q}}$ оператора

$$
\mathscr{K} f(x):=w(x) \int_{\mathbb{R}_{+}} k(x, y) f(y) u(y) d y,
$$

действующего из $L_{p}$ в $L_{q}$.

Известно (см. теорему 2 в [86]), что если $0<p<1$ и $\|\mathscr{K}\|_{L_{p} \rightarrow L_{q}}<\infty$, то $\|\mathscr{K}\|_{L_{p} \rightarrow L_{q}}=0$, поэтому изучение неравенства (1) и свойств оператора $\mathscr{K}: L_{p} \rightarrow L_{q}$ сужается на значения параметров $1 \leqslant p \leqslant \infty, 0<q \leqslant \infty$. Указанной задаче об ограниченности интегральных операторов и изучению их общих свойств посвящена обширная литература (см., например, монографии [38], [48; гл. 11], [49], [50], [72], [108]).

Следует отметить, что для знакопеременных ядер $k(x, y)$ неравенство (1) может выполняться за счет осцилляции ядра при его неограниченном росте (см., например, [46], [108]), поэтому операторы с такими ядрами изучаются в важных частных случаях. Успехи общей теории в последние двадцать лет связаны, в основном, с изучением операторов (2) с неотрицательными ядрами $k(x, y) \geqslant 0$. Несмотря на это существенное ограничение, для некоторых классов операторов Вольтерра

$$
K f(x):=w(x) \int_{0}^{x} k(x, y) f(y) u(y) d y
$$

и двойственных к ним удается получить глубокую и содержательную теорию (см. монографии [15], [52], [56]). Во-первых, решается задача нахождения двусторонней оценки $\|K\|_{L_{p} \rightarrow L_{q}} \approx F(k, u, w)$, где функционал $F(k, u, w)$ явно выражен через ядро и весовые функции, а константы эквивалентности зависят 
только от параметров $p$ и $q$. Это позволяет найти явные критерии компактности, а в отдельных случаях провести полномасштабное исследование, включая поведение характеристических чисел (аппроксимативных, энтропийных и т. д.). Эта программа с той или иной степенью общности была реализована для операторов с ядрами, удовлетворяющими условию Ойнарова: существует константа $D \geqslant 1$, не зависящая от $x, y, z$, такая, что

$$
D^{-1} k(x, y) \leqslant k(x, z)+k(z, y) \leqslant D k(x, y), \quad x \geqslant z \geqslant y \geqslant 0 .
$$

Типичными примерами таких ядер являются $(x-y)_{+}^{\alpha-1}, \alpha \geqslant 1 ; \log ^{\beta}(x / y), \beta \geqslant 0$; $\left(\int_{y}^{x} h(z) d z\right)^{\gamma}, \gamma \geqslant 0, h(z) \geqslant 0$, и различные их комбинации. Для краткости мы будем называть ядрами Ойнарова измеримые функции $k(x, y) \geqslant 0$, удовлетворяющие при $x \geqslant y \geqslant 0$ условию (4).

В разделе 1 дается краткий обзор результатов из этой области.

C начала 90-х годов прошлого столетия начал бурно развиваться $\Lambda$-анализ - круг вопросов, связанных с характеризацией ограниченности классических операторов в весовых пространствах Лоренца, определение которых мы сейчас напомним. Невозрастающей перестановкой $f^{*}$ измеримой функции $f$, заданной на $\mathbb{R}^{n}$, называется функция

$$
f^{*}(t):=\inf \left\{s>0: \operatorname{mes}\left\{x \in \mathbb{R}^{n}:|f(x)|>s\right\} \leqslant t\right\}, \quad t \in \mathbb{R}_{+} .
$$

Для фиксированного $p \in(0, \infty)$ и неотрицательной измеримой функции $v$ на $\mathbb{R}_{+}$ назовем $\Lambda$-пространством Лорениа $\Lambda^{p}(v)$ совокупность всех измеримых на $\mathbb{R}^{n}$ функций, для которых $\|f\|_{\Lambda^{p}(v)}<\infty$, где

$$
\|f\|_{\Lambda^{p}(v)}:=\left(\int_{0}^{\infty}\left[f^{*}(t)\right]^{p} v(t) d t\right)^{1 / p} .
$$

Пусть $\mathfrak{M}^{+}$обозначает конус всех неотрицательных функций из $\mathfrak{M}$, а $\mathfrak{M} \downarrow$ $\left(\mathfrak{M}^{\uparrow}\right)$ обозначает конус всех невозрастающих (неубывающих) функций из $\mathfrak{M}^{+}$. Определим

$$
L_{p, v}^{\downarrow}:=\left\{f \in \mathfrak{M}^{\downarrow}:\|f\|_{p, v}:=\left(\int_{\mathbb{R}_{+}}|f(x)|^{p} v(x) d x\right)^{1 / p}<\infty\right\}
$$

и аналогично для $L_{p, v}^{\uparrow}$ и $L_{p, v}^{+}$. Если для некоторого оператора $T: \Lambda^{p}(v) \rightarrow \Lambda^{q}(w)$ удается получить оценку

$$
[T f]^{*}(t) \ll\left[S f^{*}\right](t), \quad t>0,
$$

с положительным оператором $S$, то ограниченность $S: L_{p, v}^{\downarrow} \rightarrow L_{q, w}^{+}$влечет ограниченность $T: \Lambda^{p}(v) \rightarrow \Lambda^{q}(w)$ с неравенством для норм

$$
\|T\|_{\Lambda^{p}(v) \rightarrow \Lambda^{q}(w)} \ll\|S\|_{L_{p, v}^{\downarrow} \rightarrow L_{q, w}^{+}} .
$$

Обратное неравенство обычно ищется исходя из анализа оператора на радиальных убывающих функциях (см., например, [23]). 
Если выполняется двусторонняя оценка

$$
[T f]^{*}(t) \approx \int_{0}^{\infty} k(t, s) u(s) f^{*}(s) d s
$$

где $k(t, s) \geqslant 0$ и $u(s) \geqslant 0$, то ограниченность оператора $T$ из $\Lambda^{p}(v)$ в $\Lambda^{q}(w)$ характеризуется неравенством

$$
\left(\int_{0}^{\infty}\left(\int_{0}^{\infty} k(t, s) f(s) u(s) d s\right)^{q} w(t) d t\right)^{1 / q} \leqslant C\left(\int_{0}^{\infty}[f(t)]^{p} v(t) d t\right)^{1 / p}
$$

для всех $f \in \mathfrak{M}^{\downarrow}$. Например, известно, что максимальный оператор ХардиЛиттлвуда

$$
M f(x):=\sup _{B} \frac{1}{\operatorname{mes} B} \int_{B}|f(y)| d y,
$$

где супремум берется по всем шарам $B$ с центром в точке $x \in \mathbb{R}^{n}$, допускает двустороннюю оценку

$$
[M f]^{*}(t) \approx \frac{1}{t} \int_{0}^{t} f^{*}(s) d s
$$

поэтому его ограниченность из $\Lambda^{p}(v)$ в $\Lambda^{q}(u)$ характеризуется неравенством Харди

$$
\left(\int_{0}^{\infty}\left(\frac{1}{t} \int_{0}^{t} f(s) d s\right)^{q} w(t) d t\right)^{1 / q} \leqslant C\left(\int_{0}^{\infty}[f(t)]^{p} v(t) d t\right)^{1 / p}, \quad f \in \mathfrak{M}^{\downarrow}
$$

Аналогично, для потенциалов Рисса

$$
I_{\alpha} f(x):=\int_{\mathbb{R}^{n}} \frac{f(y) d y}{|x-y|^{n-\alpha}}, \quad 0<\alpha<n,
$$

и преобразования Гильберта

$$
\mathscr{H} f(x):=\lim _{\varepsilon \rightarrow 0} \int_{|y|>\varepsilon} \frac{f(x-y) d y}{y}
$$

выполняются двусторонние оценки

$$
\left(I_{\alpha} f\right)^{*}(t) \ll t^{\alpha / n-1} \int_{0}^{t} f^{*}(z) d z+\int_{t}^{\infty} t^{\alpha / n-1} f^{*}(z) d z \ll\left(I_{\alpha} \widetilde{f}\right)^{*}(t), \quad t>0,
$$

где $\widetilde{f}(y)=f^{*}\left(A|y|^{n}\right)$, и

$$
(\mathscr{H} f)^{*}(t) \ll C_{1}\left[t^{-1} \int_{0}^{t} f^{*}(z) d z+\int_{t}^{\infty} z^{-1} f^{*}(z) d z\right] \ll\left(\mathscr{H} f^{*}\right)^{*}(t)
$$

соответственно, поэтому их ограниченность из $\Lambda^{p}(v)$ в $\Lambda^{q}(w)$ также характеризуется неравенством типа (6).

Приведем еще один пример, когда оператор $S$ нелинеен. Для $\gamma \in(0, n)$ дробный максимальный оператор $M_{\gamma}$ определяется формулой

$$
M_{\gamma} f(x):=\sup _{B}|B|^{\gamma / n-1} \int_{B}|f(y)| d y .
$$


Тогда

$$
\left(M_{\gamma} f\right)^{*}(t) \lesssim \sup _{\tau \geqslant t} \tau^{\gamma / n-1} \int_{0}^{\tau} f^{*}(s) d s
$$

и для любой функции $\varphi \in \mathfrak{M}^{\downarrow}$ существует функция $f$ на $\mathbb{R}^{n}$ такая, что $f^{*}=\varphi$ почти всюду и

$$
\left(M_{\gamma} f\right)^{*}(t) \gtrsim \sup _{\tau \geqslant t} \tau^{\gamma / n-1} \int_{0}^{\tau} f^{*}(s) d s=: S_{\gamma} f^{*}(t) .
$$

В этом случае $\left\|M_{\gamma}\right\|_{\Lambda^{p}(v) \rightarrow \Lambda^{q}(w)} \approx\left\|S_{\gamma}\right\|_{L_{p, v}^{\downarrow} \rightarrow L_{q, w}^{+}}$. Операторы $M_{\gamma}$ играют важную роль в теории вложения пространств Соболева.

Таким образом, существенной частью $\Lambda$-анализа является задача о характеризации $\left(L_{p, v}^{\downarrow} \rightarrow L_{q, w}^{+}\right)$-неравенств для положительных операторов, которой посвящена данная работа. Попутно мы решаем аналогичную задачу для $\left(L_{p, v}^{\uparrow}-L_{q, w}^{+}\right)$-неравенств.

Отметим еще две особенности. Первая состоит в том, что выполнение неравенств на монотонных функциях не гарантирует выполнение этого же неравенства для всех неотрицательных функций. Приведем простой пример [1]: пусть весовая функция $v(x)$ имеет вид

$$
v(x)= \begin{cases}0, & 1<x<2 \\ x^{-1 / 2}, & 0<x \leqslant 1, x \geqslant 2 .\end{cases}
$$

Тогда неравенство

$$
\left(\int_{0}^{\infty}\left(\frac{1}{t} \int_{0}^{t} f(s) d s\right)^{2} v(t) d t\right)^{1 / 2} \leqslant C\left(\int_{0}^{\infty}[f(t)]^{2} v(t) d t\right)^{1 / 2}, \quad f \in \mathfrak{M}^{+},
$$

может нарушаться. Например, если $f=\chi_{(1,2)}$ (характеристическая функция интервала $(1,2))$, то правая часть равна нулю, а левая положительна. В то же время для $f \in \mathfrak{M}^{\downarrow}$ это неравенство выполнено. Поскольку $v(x) \leqslant x^{-1 / 2}$, то в силу классического неравенства Харди [40; теорема 327] имеем

$$
\begin{gathered}
\left(\int_{0}^{\infty}\left(\frac{1}{t} \int_{0}^{t} f(s) d s\right)^{2} v(t) d t\right)^{1 / 2} \leqslant\left(\int_{0}^{\infty}\left(\frac{1}{t} \int_{0}^{t} f(s) d s\right)^{2} t^{-1 / 2} d t\right)^{1 / 2} \\
\leqslant \frac{4}{3}\left(\int_{0}^{\infty}[f(t)]^{2} t^{-1 / 2} d t\right)^{1 / 2} \leqslant \frac{2^{5 / 2}}{3}\left(\int_{0}^{\infty}[f(t)]^{2} v(t) d t\right)^{1 / 2},
\end{gathered}
$$

так как $\int_{1}^{2}[f(t)]^{2} t^{-1 / 2} d t \leqslant \int_{0}^{1}[f(t)]^{2} v(t) d t$ для $f \in \mathfrak{M}^{\downarrow}$.

Таким образом, неравенство (6) может выполняться при всех $f \in \mathfrak{M}^{\downarrow}$, но нарушаться при некоторых $f \in \mathfrak{M}^{+}$. Вместе с тем для одного частного случая (6) с помощью леммы Гальперина [39], [102] удается получить эквивалентность.

Tеорема 1 [91], [76]. Пусть $1<p<\infty u 0<q<\infty$. Тогда неравенство

$$
\left(\int_{0}^{\infty}\left(\int_{0}^{t} f(s) v(s) d s\right)^{q} w(t) d t\right)^{1 / q} \leqslant C\left(\int_{0}^{\infty}[f(t)]^{p} v(t) d t\right)^{1 / p}
$$

выполняется для всех $f \in \mathfrak{M}^{+}$, если оно верно при всех $f \in \mathfrak{M}^{\downarrow}$. 
Второй особенностью является то, что неравенство (6) на всех $f \in \mathfrak{M}^{+}$легко сводится к двухвесовому неравенству заменой $f u \rightarrow f$. Для неравенств на монотонных функциях этот эффект, вообще говоря, отсутствует, поэтому наличие в неравенстве (6) трех весовых функций существенно. Кроме этого отметим, что $\left(L_{p} \rightarrow L_{q}\right)$-неравенства на монотонных функциях имеют смысл для всех значений параметров $0<p, q \leqslant \infty$.

Практически с момента возникновения $\Lambda$-анализа основным методом изучения $\left(L_{p, v}^{\downarrow} \rightarrow L_{q, w}^{+}\right)$-неравенств служит метод редукиии, т. е. сведе́ния данного неравенства на монотонных функциях к некоторому неравенству на неотрицательных функциях, которое характеризуется легче, чем исходное. Одним из универсальных средств применения метода редукции для линейных положительных операторов является приниип двойственности Сойера [87], который действует при $0<p \leqslant \infty, 1 \leqslant q \leqslant \infty$ и описанию которого посвящен раздел 2 .

Основные результаты статьи содержатся в разделе 3, где предлагается новый метод редукции $\left(L_{p, v}^{\downarrow} \rightarrow L_{q, w}^{+}\right)$- и $\left(L_{p, v}^{\uparrow} \rightarrow L_{q, w}^{+}\right)$-неравенств для положительных монотонных операторов, действующий в диапазоне $1 \leqslant p \leqslant \infty, 0<q \leqslant \infty$. Для $0<p \leqslant q<\infty$ мы также даем общую теорему для монотонных операторов с некоторым дополнительным ограничением, а в случае $0<q<p \leqslant 1$ решаем задачу для интегрального оператора Вольтерра (3) и ему двойственного с ядром, удовлетворяющим условию Ойнарова (4).

На протяжении всей статьи выражения типа $0 \cdot \infty$ полагаются равными 0 . Соотношение $A \ll B$ означает неравенство $A \leqslant c B$ с константой $c$, зависящей только от несущественных параметров. Мы будем писать $A \approx B$ вместо $A \ll$ $B \ll A$ или $A=c B$. Символ $\mathbb{Z}$ обозначает множество всех целых чисел и $\chi_{E}$ характеристическую функцию (индикатор) подмножества $E \subset \mathbb{R}_{+}$. Мы используем значки $:=$ и $=$ : для определения новых величин, а также полагаем $p^{\prime}:=p /(p-1)$ для $1<p<\infty, p^{\prime}:=1$ для $p=\infty, p^{\prime}:=\infty$ для $p=1$ и $r:=p q /(p-q)$ при $0<q<p<\infty$. Буквы $A, B, C$ с индексами, например $C_{1}, C_{2}, \ldots$, обозначают константы, возможно, с одинаковыми индексами, но различные в разных утверждениях.

\section{1. Интегральные операторы}

Пусть $u$ и $w$ - неотрицательные локально суммируемые на $\mathbb{R}_{+}$весовые функции и $k(x, y)$ - измеримое по совокупности переменных ядро. Прогресс в теории интегральных операторов за последние двадцать лет во многом связан с изучением операторов Вольтерра

$$
K f(x):=w(x) \int_{0}^{x} k(x, y) f(y) u(y) d y, \quad x>0,
$$

и неравенств

$$
\|K f\|_{q} \leqslant C\|f\|_{p},
$$

играющих важную роль в теории интегральных и дифференциальных уравнений, спектральной теории, теоремах вложения пространств Соболева и др. (см., например, монографии [10], [15], [52], [56], [64], статьи [3], [4], [118], [16]-[18], [57]-[60], [70], [71], [80]-[86], [92]-[109], [113] и др.). 
Изучение операторов (1.1) начинается с поиска критериев их ограниченности в пространствах Лебега, т. е. необходимых и/или достаточных условий выполнения неравенств (1.2), где константу $C$ мы выбираем наименьшей из возможных, т. е. равной норме $\|K\|_{L_{p} \rightarrow L_{q}}$ оператора $K$. При этом качество критерия играет ключевую роль в решении задачи о компактности оператора $K$ и более тонких исследованиях поведения его характеристических чисел. Например, простейший случай $k(x, y)=\rho(x) \geqslant 0$ изучался в рамках теории уравнений Штурма-Лиувилля в работе [19], где был получен критерий дискретности спектра. Этот и другие случаи ведут родословную с неравенств Харди [40; теоремы 327, 330], которые обобщались в работах многих авторов (см., например, исторический обзор [51]). Характерным примером является (см. [40; теоремы $329,383,402])$ неравенство (1.2) с оператором Римана-Лиувилля

$$
R_{\alpha} f(x):=w(x) \int_{0}^{x}(x-y)^{\alpha-1} f(y) u(y) d y, \quad x>0,
$$

где $\alpha>0$. Здесь случай $0<\alpha<1$ кардинально отличается от случая $\alpha \geqslant 1$. При $0<\alpha<1$ характеризация ограниченности $R_{\alpha}: L_{p} \rightarrow L_{q}$ известна лишь для частных случаев параметров суммирования и весовых функций [69], [61], [65], [79], [86]. Наиболее общий результат получен в [61] при $1<p \leqslant q<\infty$, но он имеет труднопроверяемый характер. При $\alpha \geqslant 1$ эту задачу удалось решить [95]-[98] и получить обобщения [63], [4], [71], [100], [102], [106], [107], [115] для ядер $k(x, y) \geqslant 0$, удовлетворяющих условию Ойнарова (4).

Наравне с операторами (1.1) аналогичная теория имеет место для двойственных к ним относительно билинейной формы $(f, g)=\int_{\mathbb{R}_{+}} f g$ операторов вида

$$
K^{*} g(y):=u(y) \int_{x}^{\infty} k(x, y) g(x) w(x) d x .
$$

Мы будем по мере необходимости освещать результаты и для этого класса операторов.

При крайних значениях параметров суммирования $p=1, \infty$ или $q=1, \infty$ точное значение нормы $\|K\|_{L_{p} \rightarrow L_{q}}$ находится по общей теореме [48; гл. XI, $\S 1.5$, теорема 4].

Теорема 1.1. Для оператора (1.1) верны следующие равенства:

$$
\begin{aligned}
\|K\|_{L_{1} \rightarrow L_{q}} & =\underset{t>0}{\operatorname{ess} \sup } u(t)\left\|\chi_{[t, \infty)}(\cdot) k(\cdot, t) w(\cdot)\right\|_{q}, \quad 1 \leqslant q \leqslant \infty, \\
\|K\|_{L_{\infty} \rightarrow L_{q}} & =\left(\int_{0}^{\infty}\left|w(x) \int_{0}^{x} k(x, y) u(y) d y\right|^{q} d x\right)^{1 / q}, \quad 1 \leqslant q<\infty, \\
\|K\|_{L_{p} \rightarrow L_{1}} & =\left(\int_{0}^{\infty}\left|u(y) \int_{y}^{\infty} k(x, y) w(x) d x\right|^{p^{\prime}} d y\right)^{1 / p^{\prime}}, \quad 1<p<\infty, \\
\|K\|_{L_{p} \rightarrow L_{\infty}} & =\underset{t>0}{\operatorname{ess} \sup } w(t)\left\|\chi_{[0, t]}(\cdot) k(t, \cdot) u(\cdot)\right\|_{p^{\prime}}, \quad 1<p \leqslant \infty .
\end{aligned}
$$

Для формулировки результатов об ограниченности операторов (1.1) с ядрами Ойнарова (4) нам потребуются следующие функционалы и величины:

$$
\mathbf{U}(x):=\int_{0}^{x}[u(y)]^{p^{\prime}} d y, \quad \mathbf{W}(y):=\int_{y}^{\infty}[w(x)]^{q} d x,
$$




$$
\begin{gathered}
U_{1}(x):=\int_{0}^{x} k(x, y)[u(y)]^{p^{\prime}} d y, \quad U_{p}(x):=\int_{0}^{x}[k(x, y)]^{p^{\prime}}[v(y)]^{p^{\prime}} d y \\
W_{1}(y):=\int_{y}^{\infty} k(x, y)[w(x)]^{q} d x, \quad W_{q}(y):=\int_{y}^{\infty}[k(x, y)]^{q}[w(x)]^{q} d x \\
\mathbf{A}:=\max \left(\mathbf{A}_{0}, \mathbf{A}_{1}\right),
\end{gathered}
$$

где

$$
\begin{aligned}
& \mathbf{A}_{0}:=\sup _{t>0} \mathbf{A}_{0}(t):=\sup _{t>0}\left[W_{q}(t)\right]^{1 / q}[\mathbf{U}(t)]^{1 / p^{\prime}}, \\
& \mathbf{A}_{1}:=\sup _{t>0} \mathbf{A}_{1}(t):=\sup _{t>0}[\mathbf{W}(t)]^{1 / q}\left[U_{p}(t)\right]^{1 / p^{\prime}} .
\end{aligned}
$$

Аналогично,

$$
\begin{aligned}
& \mathbb{A}:=\max \left(\mathbb{A}_{0}, \mathbb{A}_{1}\right), \\
& \mathbb{A}_{0}:=\sup _{t>0} \mathbb{A}_{0}(t):=\sup _{t>0}[\mathbf{U}(t)]^{-1 / p}\left(\int_{0}^{t}\left[U_{1}(x)\right]^{q}[w(x)]^{q} d x\right)^{1 / q}, \\
& \mathbb{A}_{1}:=\sup _{t>0} \mathbb{A}_{1}(t):=\sup _{t>0}\left[U_{p}(t)\right]^{-1 / p}\left(\int_{0}^{t}\left[U_{p}(x)\right]^{q}[w(x)]^{q} d x\right)^{1 / q} ; \\
& \mathscr{A}:=\max \left(\mathscr{A}_{0}, \mathscr{A}_{1}\right) \text {, } \\
& \mathscr{A}_{0}:=\sup _{t>0} \mathscr{A}_{0}(t):=\sup _{t>0}\left[W_{q}(t)\right]^{-1 / q^{\prime}}\left(\int_{t}^{\infty}\left[W_{q}(y)\right]^{p^{\prime}}[u(y)]^{p^{\prime}} d y\right)^{1 / p^{\prime}} \text {, } \\
& \mathscr{A}_{1}:=\sup _{t>0} \mathscr{A}_{1}(t):=\sup _{t>0}[\mathbf{W}(t)]^{-1 / q^{\prime}}\left(\int_{t}^{\infty}\left[W_{1}(y)\right]^{p^{\prime}}[u(y)]^{p^{\prime}} d y\right)^{1 / p^{\prime}} ; \\
& \mathbf{B}:=\max \left(\mathbf{B}_{0}, \mathbf{B}_{1}\right), \\
& \mathbf{B}_{0}:=\left(\int_{0}^{\infty}\left[W_{q}(t)\right]^{r / q} / d[\mathbf{U}(t)]^{r / p^{\prime}}\right)^{1 / r} \\
& \mathbf{B}_{1}:=\left(\int_{0}^{\infty}\left[U_{p}(t)\right]^{r / p^{\prime}} d\left(-[\mathbf{W}(t)]^{r / q}\right)\right)^{1 / r} ; \\
& \mathbb{B}:=\max \left(\mathbb{B}_{0}, \mathbb{B}_{1}\right), \\
& \mathbb{B}_{0}:=\left(\int_{0}^{\infty}[\mathbb{U}(t)]^{-r / p} d\left(\int_{a}^{t}\left[U_{1}(x)\right]^{q}[w(x)]^{q} d x\right)^{r / q}\right)^{1 / r}, \\
& \mathbb{B}_{1}:=\left(\int_{a}^{b}\left[U_{p}(t)\right]^{-r / p} d\left(\int_{a}^{t}\left[U_{p}(x)\right]^{q}[w(x)]^{q} d x\right)^{r / q}\right)^{1 / r} ; \\
& \mathscr{B}:=\max \left(\mathscr{B}_{0}, \mathscr{B}_{1}\right), \\
& \mathscr{B}_{0}:=\left(\int_{0}^{\infty}\left[W_{q}(t)\right]^{-r / q^{\prime}} d\left(-\left(\int_{t}^{\infty}\left[W_{q}(y)\right]^{p^{\prime}}[v(y)]^{p^{\prime}} d y\right)^{r / p^{\prime}}\right)\right)^{1 / r}, \\
& \mathscr{B}_{1}:=\left(\int_{0}^{\infty}[\mathbf{W}(t)]^{-r / q^{\prime}} d\left(-\left(\int_{0}^{\infty}\left[W_{1}(y)\right]^{p^{\prime}}[v(y)]^{p^{\prime}} d y\right)^{r / p^{\prime}}\right)\right)^{1 / r} .
\end{aligned}
$$

Используя эти функционалы, мы даем по три альтернативных критерия ограниченности операторов (1.1) с ядрами Ойнарова (4) в каждом из двух случаев соотношения параметров суммирования: $1<p \leqslant q<\infty$ и $1<q<p<\infty$. 
ТеОрема 1.2. Для оператора (1.1) с ядром $k(x, y)$, удовлетворяющим условию (4), имеют место следующие соотношения. Если $1<p \leqslant q<\infty$, то

$$
\|K\|_{L_{p} \rightarrow L_{q}} \approx \mathbf{A} \approx \mathbb{A} \approx \mathscr{A}
$$

Если $1<q<p<\infty$, mo

$$
\|K\|_{L_{p} \rightarrow L_{q}} \approx \mathbf{B} \approx \mathbb{B} \approx \mathscr{B} .
$$

Коэффициенты эквивалентности в (1.9) и (1.10) зависят только от $p, q u$ константы D в условии (4).

ЗАмЕчание 1.1. 1) При $1<p \leqslant q<\infty$ характеризация (1.2) условием $\mathscr{A}<\infty$ была получена в [4], условием $\mathbf{A}<\infty-$ в [71], условиями $\mathbf{A}<\infty$ или $\mathbb{A}<\infty-$ в [106]. При этом на ядро $k(x, y)$ накладывались некоторые дополнительные требования типа монотонности или непрерывности, которые в дальнейшем были устранены [58]. На самом деле, без ограничения общности ядро $k(x, y)$ можно считать невозрастающим по $x$ и неубывающим по $y$, иначе его можно заменить эквивалентным ядром

$$
k_{0}(x, y):=\sup _{y \leqslant z \leqslant x} \sup _{z \leqslant t \leqslant x} k(t, z)
$$

с этими свойствами (см. [58]), для которого выполняется соотношение $k(x, y) \leqslant$ $k_{0}(x, y) \leqslant D^{2} k(x, y)$.

2) В случае $1<q<p<\infty$ эквивалентность (1.10) с константой В была получена в [71] и [106]. Остальные критерии, (1.9) с константой $\mathscr{A}$ и $(1.10)$ с константами $\mathbb{B}$ и $\mathscr{B}$, найдены в [115].

$3)$ Критерии компактности оператора (1.1) с ядром $k(x, y)$, удовлетворяющим условию (4), найдены в [106].

4) Компоненты каждой из констант типа А и В в теореме 1.2, вообще говоря, независимы (см., например, [63], [97; §4], [115]), и между ними имеют место следующие соотношения:
(i) $\quad \mathbf{A}_{0}<\infty \Longleftrightarrow \mathscr{A}_{0}<\infty$,
(ii) $\mathbf{A}_{1}<\infty \Longleftrightarrow \mathbb{A}_{1}<\infty$,
(iii) $\mathbf{A}_{0}<\infty \Longrightarrow \mathbb{A}_{0}<\infty$,
(iv) $\mathbf{A}_{1}<\infty \Longrightarrow \mathscr{A}_{1}<\infty$,
(v) $\mathscr{A}_{0}<\infty \Longrightarrow \mathbb{A}_{0}<\infty$,
(vi) $\mathbb{A}_{1}<\infty \Longrightarrow \mathscr{A}_{1}<\infty$

и аналогично для остальных констант:
(i) $\mathbf{B}_{0}<\infty \Longleftrightarrow \mathscr{B}_{0}<\infty$,
(ii) $\mathbf{B}_{1}<\infty \Longleftrightarrow \mathbb{B}_{1}<\infty$,
(iii) $\mathbf{B}_{0}<\infty \Longrightarrow \mathbb{B}_{0}<\infty$,
(iv) $\mathbf{B}_{1}<\infty \Longrightarrow \mathscr{B}_{1}<\infty$,
(v) $\mathscr{B}_{0}<\infty \Longrightarrow \mathbb{B}_{0}<\infty$,
(vi) $\mathbb{B}_{1}<\infty \Longrightarrow \mathscr{B}_{1}<\infty$,

причем обратные импликации в (iii)-(vi) в каждой группе, вообще говоря, могут не иметь места.

В случае $0<q<1 \leqslant p<\infty$ хорошо известен критерий выполнения неравенства (1.2) в дискретной форме [54]. 
Теорема 1.3 [54; теорема 5]. Пусть $0<q<1 \leqslant p<\infty$. Тогда для оператора (1.1) с ядром $k(x, y)$, удовлетворяюшим условию (4), верно соотношение

$$
\|K\|_{L_{p} \rightarrow L_{q}} \approx \mathfrak{B}_{0}+\mathfrak{B}_{1}
$$

əəe

$$
\begin{aligned}
\mathfrak{B}_{0}^{r} & :=\sup _{x_{k}} \sum_{k}\left(\int_{x_{k}}^{x_{k+1}}\left[k\left(y, x_{k}\right)\right]^{q}[w(y)]^{q} d y\right)^{r / q}\left(\int_{x_{k-1}}^{x_{k}} u^{p^{\prime}}\right)^{r / p^{\prime}}, \\
\mathfrak{B}_{1}^{r} & :=\sup _{x_{k}} \sum_{k}\left(\int_{x_{k}}^{x_{k+1}} w^{q}\right)^{r / q}\left(\int_{x_{k-1}}^{x_{k}}\left[k\left(x_{k}, y\right)\right]^{p^{\prime}}[u(y)]^{p^{\prime}} d y\right)^{r / p^{\prime}},
\end{aligned}
$$

әде супремум берется по всем возрастающим последовательностям $\left\{x_{k}\right\} \subset$ $\mathbb{R}_{+}$и при $p=1$ соответствующие интегралы заменяются на супремумы подынтегральных функиий.

Недавно Д.В. Прохоровым [85] был найден интегральный критерий для неравенства (1.2).

Пусть $\alpha:=D^{q}+1$ и $\int_{t}^{\infty} w^{q}<\infty$ для всех $t \in(0, \infty)$. Определим функцию $\zeta:[0, \infty) \rightarrow[0, \infty)$ формулой (здесь $\sup \varnothing=0$ )

$$
\zeta(x):=\sup \left\{y \in(0, \infty): \int_{y}^{\infty} w^{q} \geqslant \alpha \int_{x}^{\infty} w^{q}\right\}, \quad x \in[0, \infty) .
$$

Обозначим $\zeta_{m}, m \in \mathbb{N}$, суперпозицию $m$ функций $\zeta$.

Теорема 1.4. Пусть $1 \leqslant p<\infty, 0<q<p<\infty$ и ядро $k(x, y)$ удовлетворяет условию (4). Тогда

$$
\|K\|_{L_{p} \rightarrow L_{q}} \approx \mathbb{B}+C_{\zeta}
$$

¿əe

$$
\mathbb{B}:=\left(\int_{0}^{\infty} w^{q}(x)\left[\int_{x}^{\infty} w^{q}\right]^{r / p}\left\|\chi_{\left(\zeta_{3}(x), x\right)}(\cdot) k(x, \cdot) u(\cdot)\right\|_{p^{\prime}}^{r} d x\right)^{1 / r}
$$

$u$

$$
C_{\zeta} \approx\left(\int _ { 0 } ^ { \infty } \left(w(x) k\left(x, \zeta_{2}(x)\right)^{q}\left\|\chi_{\left(0, \zeta_{2}(x)\right)} u\right\|_{p^{\prime}}^{r}\left(\int_{x}^{\infty}\left(w(s) k\left(s, \zeta_{2}(s)\right)^{q} d s\right)^{r / p} d x\right)^{1 / r} .\right.\right.
$$

ЗАМЕЧАНИЕ 1.2 .1$)$ При $k(x, y) \equiv 1$ обозначим

$$
H f(x):=w(x) \int_{0}^{x} f(y) u(y) d y, \quad x>0 .
$$

Неравенство

$$
\|H f\|_{q} \leqslant C\|f\|_{p}
$$

известно как обобщенное неравенство Харди. В этом случае $\mathbf{A}=\mathbf{A}_{0}=\mathbf{A}_{1}$, $\mathbb{A}=\mathbb{A}_{0}=\mathbb{A}_{1}, \mathscr{A}=\mathscr{A}_{0}=\mathscr{A}_{1}, \mathbf{B}_{0} \approx \mathbf{B}_{1}, \mathbb{B}=\mathbb{B}_{0}=\mathbb{B}_{1}, \mathscr{B}=\mathscr{B}_{0}=\mathscr{B}_{1}$. Кроме 
этого, утверждение теоремы 1.2 верно при $0<q<p, p>1$, а при $0<q<1=p$ имеет место следующий результат [92]:

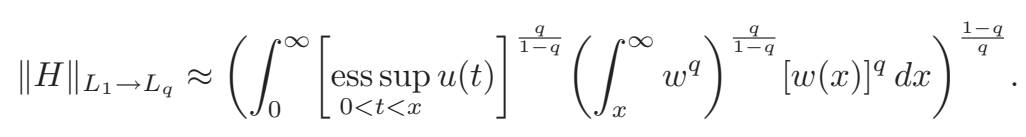

Аналогичная эквивалентность выполняется и для двойственного оператора интегрирования и их простейших модификаций.

2) Недавно обнаружилось [21], [77], что наравне с указанными выше критериями существуют семейства (шкалы) равносильных условий, зависящих от непрерывного параметра и эквивалентных $\left(L_{p}-L_{q}\right)$-ограниченности оператора $H$.

3) Разные формы критериев (1.9) и (1.10) расширяют возможности для приложений. Например, использование констант $\mathbb{A}$ и $\mathbb{B}$ для обобщенного неравенства Харди позволило найти стабильный функционал, характеризующий весовое неравенство для оператора геометрического среднего [75]. Решению этой задачи для оператора Харди-Стеклова посвящены работы [114], [74], [116].

\section{2. Принципы двойственности}

Из курса функционального анализа известно утверждение, обратное к неравенству Гёльдера:

$$
\sup _{f \in \mathfrak{M}} \frac{\int_{0}^{\infty}|f(s) g(s)| d s}{\left(\int_{0}^{\infty}|f(t)|^{p} d t\right)^{1 / p}}=\|g\|_{p^{\prime}}
$$

где $1<p<\infty$. Это равенство, которое иногда называют принципом двойственности в пространствах Лебега, позволяет редуцировать неравенства $\|T f\|_{q} \leqslant$ $C\|f\|_{p}$ с линейным оператором $T$ к неравенствам $\left\|T^{*} g\right\|_{p^{\prime}} \leqslant C\|g\|_{q^{\prime}}$ с двойственным (сопряженным) оператором $T^{*}$.

В 1990 г. Э. Сойер [87], обобщая результат работы М. Ариньо и Б. Мукенхаупта [1], где была найдена характеризация неравенства (7) при $1<p=q<\infty$, $w=v$, открыл принцип двойственности для $L_{p}$-конуса неотрицательных невозрастающих функций, что позволяет сводить неравенства типа (6) на $\mathfrak{M} \downarrow$ к неравенствам на конусе неотрицательных функций $\mathfrak{M}^{+}$, для характеризации которых имеется гораздо больше критериев. Это послужило мотивацией для широкого поля исследований весовых неравенств на конусах монотонных функций (см., например, [2], [5]-[14], [20], [22]-[37], [41]-[45], [47], [53], [55], [62], [66]-[68], [73], [76], [78], [88]-[91], [94], [99], [103], [104], [110], [111], [112], [117] и др.).

В следующем пункте мы приводим ряд утверждений, являющихся некоторым обобщением оригинального результата Сойера, и иллюстрируем границы их применения на полном описании неравенства Харди типа (7) для всех $0<p$, $q<\infty$. 
2.1. Принцип двойственности Сойера. Для локально суммируемых на $\mathbb{R}_{+}$функций $u, w \in \mathfrak{M}^{+}$обозначим $V(t):=\int_{0}^{t} v(s) d s$ и $U(t):=\int_{0}^{t} u(s) d s$ и будем считать, что $V(t)<\infty$ и $U(t)<\infty$ при всех $t>0$. Мы полагаем $V(\infty):=\lim _{t \rightarrow \infty} V(t)$ в том случае, когда этот предел существует, и аналогично определяем $U(\infty)$. Нам потребуется следующее предложение.

ПредлоЖенИЕ 2.1. Пусть $f \in \mathfrak{M}^{\downarrow}$. Тогда существует последовательность финитных суммируемых функиий $\left\{g_{n}\right\} \subset \mathfrak{M}^{+}$такая, что каждая из функциц $f_{n}(x):=\int_{x}^{\infty} g_{n}(s) d s$ возрастает по $n$ для любого $x>0$ u $f(x)=$ $\lim _{n \rightarrow \infty} \int_{x}^{\infty} g_{n}(y) d y$ для почти всех $x>0$.

ДокАзАТЕльство. Нетрудно проверить, что всем требованиям удовлетворяют функции

$$
f_{n}(x):=n \int_{x}^{x+1 / n} \chi_{(0, n)}(s) f(s) d s=: \int_{x}^{\infty} g_{n}(s) d s,
$$

где

$$
g_{n}(s)=n \begin{cases}f(s)-f(s+1 / n), & s \in(0, n-1 / n], \\ f(s), & s \in(n-1 / n, n], \\ 0, & s>n .\end{cases}
$$

Предложение доказано.

Лемма 2.1. Пусть $0<p \leqslant q<\infty$. Тогда

$$
J_{p, q}:=\sup _{f \in \mathfrak{M} \downarrow} \frac{\left(\int_{0}^{\infty}[f(s)]^{q} u(s) d s\right)^{1 / q}}{\left(\int_{0}^{\infty}[f(t)]^{p} v(t) d t\right)^{1 / p}}=\sup _{t>0} \frac{U^{1 / q}(t)}{V^{1 / p}(t)}=: A_{p, q} .
$$

ДокАзАтЕльство. Оценка $J_{p, q} \geqslant A_{p, q}$ получается, если положить под знаком супремума $f=\chi_{[0, t]}$. Поскольку $A_{p, q}=A_{1, q / p}^{1 / p}$, то заменой $f^{p} \rightarrow f$ общий случай сводится к частному, когда $1=p \leqslant q<\infty$. Применяя предложение 2.1 , теорему о монотонной сходимости и неравенство Минковского, получаем

$$
\begin{aligned}
& \left(\int_{0}^{\infty}[f(s)]^{q} u(s) d s\right)^{1 / q}=\lim _{n \rightarrow \infty}\left(\int_{0}^{\infty}\left(\int_{s}^{\infty} g_{n}(t) d t\right)^{q} u(s) d s\right)^{1 / q} \\
& \quad \leqslant \lim _{n \rightarrow \infty} \int_{0}^{\infty} U^{1 / q}(t) g_{n}(t) d t \leqslant A_{1, q} \lim _{n \rightarrow \infty} \int_{0}^{\infty} V(t) g_{n}(t) d t \\
& \quad=A_{1, q} \lim _{n \rightarrow \infty} \int_{0}^{\infty}\left(\int_{0}^{t} v(s) d s\right) g_{n}(t) d t=A_{1, q} \lim _{n \rightarrow \infty} \int_{0}^{\infty}\left(\int_{s}^{\infty} g_{n}(t) d t\right) v(s) d s \\
& \quad=A_{1, q} \int_{0}^{\infty} f(s) v(s) d s .
\end{aligned}
$$

Лемма доказана.

Лемма 2.2. Пусть $0<q<p<\infty, 1 / r:=1 / q-1 / p$. Тогда

$$
J_{p, q} \approx\left(\int_{0}^{\infty}\left(\int_{t}^{\infty} \frac{u(s) d s}{V(s)}\right)^{r / q} v(t) d t\right)^{1 / r}=: B_{p, q}
$$


ДокАЗАТЕЛЬСтво. Поскольку $B_{p, q}=B_{p / q, 1}^{1 / q}$, то заменой $f^{q} \rightarrow f$ общий случай сводится к частному, когда $1=q<p<\infty$, т. е. достаточно показать, что

$$
J_{p, 1} \approx\left(\int_{0}^{\infty}\left(\int_{t}^{\infty} \frac{u(s) d s}{V(s)}\right)^{p^{\prime}} v(t) d t\right)^{1 / p^{\prime}}=B_{p, 1} .
$$

Пусть $f \in \mathfrak{M}^{\downarrow}$. По неравенству Гёльдера имеем

$$
\begin{aligned}
\int_{0}^{\infty} f(s) u(s) d s & =\int_{0}^{\infty} f(s) \frac{u(s)}{V(s)}\left(\int_{0}^{s} v(t) d t\right) d s \\
& =\int_{0}^{\infty} v(t)\left(\int_{t}^{\infty} \frac{f(s) u(s) d s}{V(s)}\right) d t \leqslant \int_{0}^{\infty} v(t) f(t)\left(\int_{t}^{\infty} \frac{u(s) d s}{V(s)}\right) d t \\
& \leqslant B_{p, 1}\left(\int_{0}^{\infty}[f(t)]^{p} v(t) d t\right)^{1 / p}
\end{aligned}
$$

поэтому $J_{p, 1} \leqslant B_{p, 1}$. Для того чтобы доказать обратное неравенство, рассмотрим тестовую функцию

$$
f_{0}(t):=\left(\int_{t}^{\infty} \frac{u(s) d s}{V(s)}\right)^{1 /(p-1)} .
$$

Тогда, интегрируя по частям, находим

$$
\begin{aligned}
\int_{0}^{\infty}\left[f_{0}(t)\right]^{p} v(t) d t & =\int_{0}^{\infty}\left(\int_{t}^{\infty} \frac{u(s) d s}{V(s)}\right)^{p /(p-1)} v(t) d t \\
& =p^{\prime} \int_{0}^{\infty}\left(\int_{t}^{\infty} \frac{u(s) d s}{V(s)}\right)^{1 /(p-1)} u(t) d t=p^{\prime} \int_{0}^{\infty} f_{0}(t) u(t) d t
\end{aligned}
$$

Отсюда, используя равенство второго и последнего выражений, получаем

$$
J_{p, 1} \geqslant \frac{\int_{0}^{\infty} f_{0}(t) u(t) d t}{\left(\int_{0}^{\infty}\left[f_{0}(t)\right]^{p} v(t) d t\right)^{1 / p}}=\left(p^{\prime}\right)^{-1 / p}\left(\int_{0}^{\infty} f_{0}(t) u(t) d t\right)^{1 / p^{\prime}}=\frac{1}{p^{\prime}} B_{p, 1} .
$$

Лемма доказана.

ЗАмечАниЕ 2.1. Можно показать на примерах, что неравенство

$$
\frac{1}{p^{\prime}} B_{p, 1} \leqslant J_{p, 1} \leqslant B_{p, 1}
$$

полученное при доказательстве леммы 2.2, точное (см. [76]).

Лемма 2.2 имеет полезный альтернативный вариант.

Лемма 2.3. Пусть $0<q<p<\infty, 1 / r:=1 / q-1 / p$. Тогда

$$
J_{p, q} \approx\left(\int_{0}^{\infty}\left(\frac{U(t)}{V(t)}\right)^{r / q} v(t) d t\right)^{1 / r}+\frac{U^{1 / q}(\infty)}{V^{1 / p}(\infty)}=: \mathbf{B}_{p, q}
$$


ДокАЗАТЕЛЬСтво. Поскольку $\mathbf{B}_{p, q}=\mathbf{B}_{p / q, 1}^{1 / q}$, то снова заменой $f^{q} \rightarrow f$ общий случай сводится к частному, когда $1=q<p<\infty$, т. е. достаточно показать, что

$$
J_{p, 1} \approx\left(\int_{0}^{\infty}\left(\frac{U(t)}{V(t)}\right)^{p^{\prime}} v(t) d t\right)^{1 / p^{\prime}}+\frac{U(\infty)}{V^{1 / p}(\infty)}=\mathbf{B}_{p, 1} .
$$

Сужая супремум в выражении $J_{p, 1}$ на постоянные функции, находим, что

$$
J_{p, 1} \geqslant \frac{U(\infty)}{V^{1 / p}(\infty)}
$$

Аналогично, сужая супремум в выражении $J_{p, 1}$ на функции вида $f(s)=\int_{s}^{\infty} h$, получим

$$
J_{p, 1} \geqslant \sup _{h \in \mathfrak{M}^{+}} \frac{\int_{0}^{\infty}\left(\int_{s}^{\infty} h(t) d t\right) u(s) d s}{\left(\int_{0}^{\infty}\left(\int_{s}^{\infty} h(t) d t\right)^{p} v(t) d t\right)^{1 / p}}=\sup _{h \in \mathfrak{M}^{+}} \frac{\int_{0}^{\infty} h(t) U(t) d t}{\left(\int_{0}^{\infty}\left(\int_{s}^{\infty} h(t) d t\right)^{p} v(t) d t\right)^{1 / p}}
$$

Из неравенства Харди вытекает, что

$$
\left(\int_{0}^{\infty}\left(\int_{t}^{\infty} h\right)^{p} v(t) d t\right)^{1 / p} \ll\left(\int_{0}^{\infty}[h V]^{p}[v]^{1-p}\right)^{1 / p}
$$

Следовательно,

$$
J_{p, 1} \gg \sup _{h \in \mathfrak{M}^{+}} \frac{\int_{0}^{\infty} h(t) U(t) d t}{\left(\int_{0}^{\infty}[h(t) V(t)]^{p}[v(t)]^{1-p} d t\right)^{1 / p}},
$$

и, применяя принцип двойственности в $L_{p}$, получим

$$
J_{p, 1} \gg\left(\int_{0}^{\infty}\left(\frac{U(t)}{V(t)}\right)^{p^{\prime}} v(t) d t\right)^{1 / p^{\prime}}
$$

Из (2.9) и (2.11) следует оценка снизу $J_{p, 1} \gg \mathbf{B}_{p, 1}$. Для доказательства оценки сверху, интегрируя по частям, запишем

$$
\int_{x}^{\infty} \frac{u(s) d s}{V(s)}=\int_{x}^{\infty} \frac{d U(s)}{V(s)} \leqslant \frac{U(\infty)}{V(\infty)}+\int_{t}^{\infty} \frac{U(s) v(s) d s}{V^{2}(s)} .
$$

Пусть $f \in \mathfrak{M}^{\downarrow}$. Тогда, продолжая цепочку (2.5), находим, что

$$
\int_{0}^{\infty} f(t) u(t) d t \leqslant\left(\int_{0}^{\infty} f(x) v(x) d x\right) \frac{U(\infty)}{V(\infty)}+\int_{0}^{\infty} f(x) v(x)\left(\int_{x}^{\infty} \frac{U(s) v(s) d s}{V^{2}(s)}\right) d x .
$$

Применяя неравенство Гёльдера, получим

$$
\begin{aligned}
& \int_{0}^{\infty} f(t) u(t) d t \\
& \quad \leqslant\left(\int_{0}^{\infty} f^{p} v\right)^{1 / p}\left(\frac{U(\infty)}{V^{1 / p}(\infty)}+\left(\int_{0}^{\infty} v(x)\left(\int_{x}^{\infty} \frac{U(s) v(s) d s}{V^{2}(s)}\right)^{p^{\prime}} d x\right)^{1 / p^{\prime}}\right) .
\end{aligned}
$$


По неравенству Харди

$$
\left(\int_{0}^{\infty} v(x)\left(\int_{x}^{\infty} \frac{U(s) v(s) d s}{V^{2}(s)}\right)^{p^{\prime}} d x\right)^{1 / p^{\prime}} \ll\left(\int_{0}^{\infty} \frac{U^{p^{\prime}}(s) v(s) d s}{V^{p^{\prime}}(s)}\right)^{1 / p^{\prime}},
$$

поэтому

$$
\frac{\int_{0}^{\infty} f u}{\left(\int_{0}^{\infty} f^{p} v\right)^{1 / p}} \ll\left(\int_{0}^{\infty} \frac{U^{p^{\prime}}(s) v(s) d s}{V^{p^{\prime}}(s)}\right)^{1 / p^{\prime}}+\frac{U(\infty)}{V^{1 / p}(\infty)},
$$

и в силу произвольности $f \in \mathfrak{M}^{\downarrow}$ отсюда вытекает соотношение $J_{p, 1} \ll \mathbf{B}_{p, 1}$. Лемма доказана.

ЗАмЕЧАНИЕ 2.2. Леммы 2.1-2.3 при $q=1$ составляют принцип двойственности для конуса убывающих функций. Этот прицип позволяет характеризовать неравенство (6) при $q \geqslant 1$.

Сначала сформулируем случай $q=1$.

Теорема 2.1. Пусть $0<p<\infty$. Тогда для наименъшей константы $C$ в неравенстве

$$
\int_{0}^{\infty} u(x) \int_{0}^{\infty} k(x, y) f(y) w(y) d y d x \leqslant C\left(\int_{0}^{\infty}[f(x)]^{p} v(x) d x\right)^{1 / p}, \quad f \in \mathfrak{M}^{\downarrow},
$$

выполняются: при $0<p \leqslant 1$ равенство

$$
C=\sup _{t>0} \frac{\int_{0}^{t} w(y) \int_{0}^{\infty} k(x, y) u(x) d x d y}{V^{1 / p}(t)},
$$

а при $1<p<\infty$ двусторонние ощенки

$$
\begin{gathered}
C \approx\left(\int_{0}^{\infty}\left(\int_{t}^{\infty} \frac{w(y) \int_{0}^{\infty} k(x, y) u(x) d x d y}{V(y)}\right)^{p^{\prime}} v(t) d t\right)^{1 / p^{\prime}} \\
C \approx\left(\int_{0}^{\infty}\left(\frac{\int_{0}^{t} w(y) \int_{0}^{\infty} k(x, y) u(x) d x d y}{V(t)}\right)^{p^{\prime}} v(t) d t\right)^{1 / p^{\prime}} \\
+\frac{\int_{0}^{\infty} w(y) \int_{0}^{\infty} k(x, y) u(x) d x d y}{V^{1 / p}(\infty)} .
\end{gathered}
$$

ДокАЗАТЕЛьство. Следует применить леммы 2.1-2.3 при $q=1$.

Для $1<q<\infty$ неравенство (6) на функциях из $\mathfrak{M}^{\downarrow}$ сводится к неравенствам на функциях из $\mathfrak{M}^{+}$. Имеет место следующая теорема.

Теорема 2.2. Пустъ $0<p<\infty, 1<q<\infty$. Тогда для наименъшей константы $С$ в неравенстве

$$
\begin{aligned}
& \left(\int_{0}^{\infty}\left(\int_{0}^{\infty} k(x, y) f(y) w(y) d y\right)^{q} u(x) d x\right)^{1 / q} \\
& \quad \leqslant C\left(\int_{0}^{\infty}[f(x)]^{p} v(x) d x\right)^{1 / p}, \quad f \in \mathfrak{M}^{\downarrow}
\end{aligned}
$$


выполняются: при $0<p \leqslant 1$ равенство

$$
C=\sup _{t>0} \frac{\left(\int_{0}^{\infty}\left(\int_{0}^{t} k(x, y) w(y) d y\right)^{q} u(x) d x\right)^{1 / q}}{V^{1 / p}(t)}
$$

а при $1<p<\infty$ двусторонние ощенки

$$
\begin{aligned}
C \approx \sup _{g \in \mathfrak{M}^{+}} & \frac{\left(\int_{0}^{\infty}\left(\int_{0}^{\infty}\left(\int_{t}^{\infty} \frac{k(x, y) w(y) d y}{V(y)}\right) g(t) v(t) d t\right)^{q} u(x) d x\right)^{1 / q}}{\left(\int_{0}^{\infty} g^{p} v\right)^{1 / p}}, \\
C \approx & \frac{\left(\int_{0}^{\infty}\left(\int_{0}^{\infty} k(x, y) w(y) d y\right)^{q} u(x) d x\right)^{1 / q}}{V^{1 / p}(\infty)} \\
& \quad+\sup _{g \in \mathfrak{M}^{+}} \frac{\left(\int_{0}^{\infty}\left(\int_{0}^{\infty}\left(\int_{0}^{t} k(x, y) w(y) d y\right) \frac{g(t) v(t) d t}{V(t)}\right)^{q} u(x) d x\right)^{1 / q}}{\left(\int_{0}^{\infty} g^{p} v\right)^{1 / p}} .
\end{aligned}
$$

ДокАЗАТЕЛЬСтво опирается на леммы 2.1-2.3 и принцип двойственности (2.1) в $L_{p}$.

ЗАмЕчание 2.3. Формула (2.17) сохраняется при $0<p \leqslant q<\infty, 0<p \leqslant 1$ (см. [99], [104], [53], [66]). Более того, имеет место в некотором смысле двойственный результат.

ТеОрема 2.3. Пусть $1 \leqslant p \leqslant q<\infty$. Тогда для наименъшей константы $C$ в неравенстве

$$
\left(\int_{0}^{\infty} f^{q} u\right)^{1 / q} \leqslant C\left(\int_{0}^{\infty}\left(\int_{0}^{\infty} k(x, y) f(y) w(y) d y\right)^{p} v(x) d x\right)^{1 / p}, \quad f \in \mathfrak{M}^{\downarrow}
$$

выполняется равенство

$$
C=\sup _{t>0} \frac{U^{1 / q}(t)}{\left(\int_{0}^{\infty}\left(\int_{0}^{t} k(x, y) w(y) d y\right)^{p} v(x) d x\right)^{1 / p}}=: \mathscr{A}_{p, q} .
$$

ДокАЗАТЕЛЬство. Подставляя в неравенство (2.20) тестовую функцию $f_{t}:=\chi_{[0, t]}$ и переходя к супремуму по $t>0$, получаем $C \geqslant \mathscr{A}_{p, q}$. Для доказательства обратного неравенства воспользуемся предложением 2.1. Применяя неравенство Минковского, имеем

$$
\begin{aligned}
& \left(\int_{0}^{\infty} f^{q} u\right)^{1 / q}=\lim _{n \rightarrow \infty}\left(\int_{0}^{\infty}\left(\int_{x}^{\infty} g_{n}\right)^{q} u(x) d x\right)^{1 / q} \\
& \quad=\lim _{n \rightarrow \infty}\left(\int_{0}^{\infty}\left(p \int_{x}^{\infty}\left(\int_{t}^{\infty} g_{n}\right)^{p-1} g_{n}(t) d t\right)^{q / p} u(x) d x\right)^{1 / q} \\
& \quad \leqslant \lim _{n \rightarrow \infty}\left(\int_{0}^{\infty} p\left(\int_{t}^{\infty} g_{n}\right)^{p-1} g_{n}(t) U^{p / q}(t) d t\right)^{1 / p} \\
& \leqslant \mathscr{A}_{p, q} \lim _{n \rightarrow \infty}\left(\int_{0}^{\infty} p\left(\int_{t}^{\infty} g_{n}\right)^{p-1} g_{n}(t) \int_{0}^{\infty}\left(\int_{0}^{t} k(x, y) w(y) d y\right)^{p} v(x) d x d t\right)^{1 / p}
\end{aligned}
$$




$$
\begin{aligned}
& =\mathscr{A}_{p, q} \lim _{n \rightarrow \infty}\left(p \int_{0}^{\infty} v(x) \int_{0}^{\infty}\left(\int_{0}^{t} k(x, y) w(y) d y\right)^{p}\left(\int_{t}^{\infty} g_{n}\right)^{p-1} g_{n}(t) d t d x\right)^{1 / p} \\
& \leqslant \mathscr{A}_{p, q} \lim _{n \rightarrow \infty}\left(\int_{0}^{\infty}\left(\int_{0}^{\infty} k(x, y) w(y)\left(\int_{y}^{\infty} g_{n}\right) d y\right)^{p} v(x) d x\right)^{1 / p} \\
& =\mathscr{A}_{p, q}\left(\int_{0}^{\infty}\left(\int_{0}^{\infty} k(x, y) f(y) w(y) d y\right)^{p} v(x) d x\right)^{1 / p} .
\end{aligned}
$$

Теорема доказана.

Tеорема 2.4. Пусть $0<p \leqslant 1 \leqslant q<\infty u k_{i}(x, y) \geqslant 0, i=1,2$. Тогда для наименъшей из возможных констант $C$ в неравенстве

$$
\begin{aligned}
& \left(\int_{0}^{\infty}\left(\int_{0}^{\infty} k_{1}(x, y) f(y) d y\right)^{q} u(x) d x\right)^{1 / q} \\
& \quad \leqslant C\left(\int_{0}^{\infty}\left(\int_{0}^{\infty} k_{2}(x, y) f(y) d y\right)^{p} v(x) d x\right)^{1 / p}, \quad f \in \mathfrak{M}^{\downarrow}
\end{aligned}
$$

выполняется равенство $C=\mathbf{A}_{p, q}$, әде

$$
\mathbf{A}_{p, q}:=\sup _{t>0}\left(\int_{0}^{\infty}\left(\int_{0}^{t} k_{1}(x, y) d y\right)^{q} u(x) d x\right)^{1 / q}\left(\int_{0}^{\infty}\left(\int_{0}^{t} k_{2}(x, y) d y\right)^{p} v(x) d x\right)^{-1 / p} .
$$

ДокАЗАтельство. Оценка $\mathbf{A}_{p, q} \leqslant C$ получается подстановкой тестовой функции $f=\chi_{[0, t]}$. Для доказательства обратного неравенства мы используем представление $f(y)=\int_{y}^{\infty} h(s) d s$ и дважды неравенство Минковского:

$$
\begin{aligned}
\left(\int_{0}^{\infty}\left(\int_{0}^{\infty} k_{1}(x, y) f(y) d y\right)^{q} u(x) d x\right)^{1 / q} \\
=\left(\int_{0}^{\infty}\left(\int_{0}^{\infty} k_{1}(x, y)\left(\int_{y}^{\infty} h(t) d t\right) d y\right)^{q} u(x) d x\right)^{1 / q} \\
=\left(\int_{0}^{\infty}\left(\int_{0}^{\infty} h(t) \int_{0}^{t} k_{1}(x, y) d y d t\right)^{q} u(x) d x\right)^{1 / q} \\
\leqslant \int_{0}^{\infty} h(t)\left(\int_{0}^{\infty}\left(\int_{0}^{t} k_{1}(x, y) d y\right)^{q} u(x) d x\right)^{1 / q} d t \\
\leqslant \mathbf{A}_{p, q} \int_{0}^{\infty} h(t)\left(\int_{0}^{\infty}\left(\int_{0}^{t} k_{2}(x, y) d y\right)^{p} v(x) d x\right)^{1 / p} d t \\
\leqslant \mathbf{A}_{p, q}\left(\int_{0}^{\infty}\left(\int_{0}^{\infty}\left(\int_{0}^{t} k_{2}(x, y) d y\right)^{p} h(t) d t\right)^{p(x) d x)^{1 / p}}\right. \\
=\mathbf{A}_{p, q}\left(\int_{0}^{\infty}\left(\int_{0}^{\infty} k_{2}(x, y)\left(\int_{y}^{\infty} h(t) d t\right)^{p} d y\right)^{1 / p} v(x) d x\right)^{p} \\
=\mathbf{A}_{p, q}\left(\int_{0}^{\infty}\left(\int_{0}^{\infty} k_{2}(x, y) f(y) d y\right)^{p} v(x) d x\right)^{1 / p} \cdot
\end{aligned}
$$

Требуемый результат получаем, применяя предложение 2.1. Теорема доказана. 
2.2. Неравенство Харди на монотонных функциях. Если в (6) положить $k(x, y)=\chi_{[0, x]}(y)$, то мы получим трехвесовое неравенство Харди

$$
\left(\int_{0}^{\infty}\left(\int_{0}^{x} f(y) u(y) d y\right)^{q} w(x) d x\right)^{1 / q} \leqslant C\left(\int_{0}^{\infty}[f(x)]^{p} v(x) d x\right)^{1 / p}, \quad f \in \mathfrak{M}^{\downarrow},
$$

хорошо известное многочисленными применениями.

$$
\text { Напомним обозначения: } U(t):=\int_{0}^{t} u, V(t):=\int_{0}^{t} v, W(t):=\int_{0}^{t} w \text {. }
$$

Теорема 2.5. Для наименъшей константы $C$ в неравенстве (2.22) имеют место двусторонние оценки:

(a) если $1<p \leqslant q<\infty$, то $C \approx A_{0}+A_{1}$, где

$$
\begin{aligned}
& A_{0}=\sup _{t>0} A_{0}(t):=\sup _{t>0}\left(\int_{0}^{t} U^{q} w\right)^{1 / q} V^{-1 / p}(t), \\
& A_{1}:=\sup _{t>0}\left(\int_{t}^{\infty} w\right)^{1 / q}\left(\int_{0}^{t}\left(\frac{U}{V}\right)^{p^{\prime}} v\right)^{1 / p^{\prime}} ;
\end{aligned}
$$

(b) если $0<q<p<\infty, 1<p<\infty$, то $C \approx B_{0}+B_{1}$, где

$$
\begin{aligned}
& B_{0}=B_{0}(p, q):=\left(\int_{0}^{\infty} V^{-r / p}(t)\left(\int_{0}^{t} U^{q} w\right)^{r / p} U^{q}(t) w(t) d t\right)^{1 / r} \\
& B_{1}=B_{1}(p, q):=\left(\int_{0}^{\infty}\left(\int_{t}^{\infty} w\right)^{r / p}\left(\int_{0}^{t}\left(\frac{U}{V}\right)^{p^{\prime}} v\right)^{r / p^{\prime}} w(t) d t\right)^{1 / r}
\end{aligned}
$$

(c) если $0<q<p \leqslant 1$, mо $C \approx B_{0}+\mathscr{B}_{1}$, где

$$
\mathscr{B}_{1}=\mathscr{B}_{1}(p, q):=\left(\int_{0}^{\infty}\left(\underset{s \in[0, t]}{\operatorname{esssup}} \frac{U^{p}(s)}{V(s)}\right)^{r / p}\left(\int_{t}^{\infty} w\right)^{r / p} w(t) d t\right)^{1 / r} ;
$$

(d) если $0<p \leqslant q<\infty, 0<p \leqslant 1$, mо $C=\mathscr{A}_{1}$, где

$$
\left.\mathscr{A}_{1}:=\sup _{t>0} V^{-1 / p}(t)\left(\int_{0}^{\infty} U^{q}(\min \{s, t\}) w(s) d s\right)\right)^{1 / q} \text {. }
$$

ДоказАТЕЛьство. Пункт (d) следует из (2.17) с учетом замечания 2.3.

В силу монотонности $f$ из $(2.22)$ следует неравенство

$$
\left(\int_{0}^{\infty}[f U]^{q} w\right)^{1 / q} \leqslant C\left(\int_{0}^{\infty}[f]^{p} v\right)^{1 / p}, \quad f \in \mathfrak{M}^{\downarrow}
$$

откуда ввиду лемм 2.1 и 2.3 следуют оценки снизу $A_{0} \leqslant C$ при $0<p \leqslant q<\infty$ и

$$
\mathscr{B}_{0}:=\left(\int_{0}^{\infty}\left(\int_{0}^{t} U^{q} w\right)^{r / q} V^{-r / q}(t) v(t) d t\right)^{1 / r} \ll C, \quad A_{0}(\infty) \leqslant C
$$

при $0<q<p<\infty$. Интегрированием по частям находим

$$
B_{0}=\left(\frac{q}{r} \frac{\left(\int_{0}^{\infty} U^{q} w\right)^{r / q}}{V^{r / p}(\infty)}+\frac{q}{p} \mathscr{B}_{0}^{r}\right)^{1 / r} \approx A_{0}(\infty)+\mathscr{B}_{0},
$$


откуда легко следует неравенство $B_{0} \leqslant C$. Заметим, что при любом $t>0$

$$
\begin{aligned}
B_{0}^{r} & \geqslant \int_{0}^{t} V^{-r / p}(s)\left(\int_{0}^{s} U^{q} w\right)^{r / p} U^{q}(s) w(s) d s=\frac{q}{r} \int_{0}^{t} V^{-r / p}(s) d\left(\int_{0}^{s} U^{q} w\right)^{r / q} \\
& \geqslant \frac{q}{r} V^{-r / p}(t) \int_{0}^{t} d\left(\int_{0}^{s} U^{q} w\right)^{r / q}=\frac{q}{r} V^{-r / p}(t)\left(\int_{0}^{t} U^{q} w\right)^{r / q}=\frac{q}{r} A_{0}^{r}(t),
\end{aligned}
$$

откуда

$$
B_{0} \gg A_{0} \geqslant A_{0}(\infty)
$$

Для доказательства остальных утверждений пункта (a) и пункта (b) при $q \geqslant 1$ следует применить теоремы 2.1, 2.2 и критерии выполнения неравенств Харди для неотрицательных функций. Обратимся к подробностям. По формуле (2.19) находим

$$
\begin{gathered}
C \approx A_{0}(\infty)+\sup _{g \in \mathfrak{M}^{+}} \frac{\left(\int_{0}^{\infty}\left(\int_{0}^{x}(U g v / V)+U(x) \int_{x}^{\infty}(g v / V)\right)^{q} w(x) d x\right)^{1 / q}}{\left(\int_{0}^{\infty} g^{p} v\right)^{1 / p}} \\
\approx A_{0}(\infty)+\sup _{g \in \mathfrak{M}^{+}} \frac{\left(\int_{0}^{\infty}\left(\int_{0}^{x}(U g v / V)\right)^{q} w(x) d x\right)^{1 / q}}{\left(\int_{0}^{\infty} g^{p} v\right)^{1 / p}} \\
\quad+\sup _{g \in \mathfrak{M}^{+}} \frac{\left(\int_{0}^{\infty}\left(U(x) \int_{x}^{\infty}(g v / V)\right)^{q} w(x) d x\right)^{1 / q}}{\left(\int_{0}^{\infty} g^{p} v\right)^{1 / p}} .
\end{gathered}
$$

Отсюда при $1<p \leqslant q<\infty$, используя критерии для неравенств Харди, находим

$$
C \approx A_{0}(\infty)+A_{1}+\sup _{t>0}\left(\int_{0}^{t} U^{q} w\right)^{1 / q}\left(\int_{t}^{\infty} \frac{v}{V^{p^{\prime}}}\right)^{1 / p^{\prime}}
$$

откуда следуют оценки $C \gg A_{1}, C \ll A_{0}+A_{1}$, и пункт (а) доказан.

При $1<q<p<\infty$ из $(2.24)$ и критериев для неравенств Харди следует, что

$$
C \approx A_{0}(\infty)+B_{1}+\left(\int_{0}^{\infty}\left(\int_{0}^{t} U^{q} w\right)^{r / p}\left(\int_{t}^{\infty} \frac{v}{V^{p^{\prime}}}\right)^{r / p^{\prime}} U^{q}(t) w(t) d t\right)^{1 / r},
$$

поэтому $C \gg B_{1}, C \ll B_{0}+B_{1}$. При $1=q<p<\infty$ из (2.15) находим

$$
\begin{aligned}
C \approx A_{0}(\infty)+\left(\int_{0}^{\infty} V^{-p^{\prime}}(t)\left(\int_{0}^{t} u(y)\left(\int_{y}^{\infty} w\right) d y\right)^{p^{\prime}} v(t) d t\right)^{1 / p^{\prime}} \\
\approx A_{0}(\infty)+\left(\int_{0}^{\infty} V^{-p^{\prime}}(t)\left(\int_{0}^{t} U w\right)^{p^{\prime}} v(t) d t\right)^{1 / p^{\prime}} \\
\quad+\left(\int_{0}^{\infty}\left(\frac{U(t)}{V(t)}\right)^{p^{\prime}}\left(\int_{t}^{\infty} w\right)^{p^{\prime}} v(t) d t\right)^{1 / p^{\prime}} \\
=A_{0}(\infty)+\left(p^{\prime}\right)^{1 / p^{\prime}} B_{1}+\left(\int_{0}^{\infty} V^{-p^{\prime}}(t)\left(\int_{0}^{t} U w\right)^{p^{\prime}} v(t) d t\right)^{1 / p^{\prime}} .
\end{aligned}
$$

Отсюда следуют оценки $C \gg B_{1}, C \ll B_{0}+B_{1}$, и пункт (b) при $q \geqslant 1$ доказан. 
Рассмотрим случай (b) при $0<q<1<p<\infty$. Напомним, что оценка снизу $C \gg B_{0}$ уже получена. По теореме 3.1 , доказанной ниже, неравенство $(2.22)$ эквивалентно неравенству

$$
\left(\int_{0}^{\infty}\left(\int_{0}^{x}\left(\int_{y}^{\infty} h(z) u(z) d z\right)\right)^{q} w(x) d x\right)^{1 / q} \leqslant C\left(\int_{0}^{\infty} h^{p} V^{p} v^{1-p}\right)^{1 / p}, \quad h \in \mathfrak{M}^{+}
$$

которое, в свою очередь, распадается на два неравенства Харди

$$
\left(\int_{0}^{\infty}\left(\int_{x}^{\infty} h\right)^{q} U^{q}(x) w(x) d x\right)^{1 / q} \leqslant C_{1}\left(\int_{0}^{\infty} h^{p} V^{p} v^{1-p}\right)^{1 / p}, \quad h \in \mathfrak{M}^{+},
$$

и

$$
\left(\int_{0}^{\infty}\left(\int_{0}^{x} h U\right)^{q} w(x) d x\right)^{1 / q} \leqslant C_{2}\left(\int_{0}^{\infty} h^{p} V^{p} v^{1-p}\right)^{1 / p}, \quad h \in \mathfrak{M}^{+},
$$

так, что $C \approx C_{1}+C_{2}$. Из критериев для неравенств Харди следует, что $C_{1} \ll B_{0}$ и $C_{2} \approx B_{1}$, поэтому $C \approx B_{0}+B_{1}$.

Для доказательства пункта (c) нам потребуется модификация формулы (1.18): при $0<q<1$ для наименьшей константы $C$ в неравенстве

$$
\left(\int_{0}^{\infty}\left(\int_{0}^{x} h(y) d y\right)^{q} w(x) d x\right)^{1 / q} \leqslant C \int_{0}^{\infty} h v, \quad h \in \mathfrak{M}^{+}
$$

выполняется двусторонняя оценка

$$
C \approx\left(\int_{0}^{\infty}\left(\operatorname{esssup}_{s \in[0, t]} \frac{1}{v(s)}\right)^{\frac{q}{1-q}}\left(\int_{t}^{\infty} w\right)^{\frac{q}{1-q}} w(t) d t\right)^{\frac{1-q}{q}}=: \mathscr{B} .
$$

Сначала рассмотрим случай $0<q<1=p$. Допустим, что неравенство (2.22) выполнено. Тогда с той же константой $C$ оно выполнено для всех функций $f$ вида $f(x)=\int_{x}^{\infty} h$, где $h \in \mathfrak{M}^{+}$. Имеем

$$
\left(\int_{0}^{\infty}\left(\int_{0}^{x}\left(\int_{y}^{\infty} h\right) u(y) d y\right)^{q} w(x) d x\right)^{1 / q} \leqslant C \int_{0}^{\infty}\left(\int_{x}^{\infty} h\right) v(x) d x, \quad h \in \mathfrak{M}^{+} .
$$

Отсюда следует неравенство

$$
\left(\int_{0}^{\infty}\left(\int_{0}^{x} h U\right)^{q} w(x) d x\right)^{1 / q} \leqslant C \int_{0}^{\infty} h V, \quad h \in \mathfrak{M}^{+} .
$$

Применяя (2.28), получим $C \gg \mathscr{B}_{1}(1, q)$. Для доказательства обратной оценки применим предложение 2.1 , лемму 2.3 и оценки (2.23) и (2.28). Имеем

$$
\begin{aligned}
& \left(\int_{0}^{\infty}\left(\int_{0}^{x} f u\right)^{q} w(x) d x\right)^{1 / q}=\lim _{n \rightarrow \infty}\left(\int_{0}^{\infty}\left(\int_{0}^{x}\left(\int_{y}^{\infty} h_{n}\right) u(y) d y\right)^{q} w(x) d x\right)^{1 / q} \\
& \ll \lim _{n \rightarrow \infty}\left(\int_{0}^{\infty}\left(\int_{0}^{x} h_{n} U\right)^{q} w(x) d x\right)^{1 / q}+\lim _{n \rightarrow \infty}\left(\int_{0}^{\infty}\left(\int_{x}^{\infty} h_{n}\right)^{q} U^{q}(x) w(x) d x\right)^{1 / q}
\end{aligned}
$$




$$
\begin{aligned}
& \ll \mathscr{B}_{1}(1, q) \lim _{n \rightarrow \infty} \int_{0}^{\infty} h_{n} V+B_{0} \lim _{n \rightarrow \infty} \int_{0}^{\infty}\left(\int_{x}^{\infty} h_{n}\right) v(x) d x \\
& =\left(\mathscr{B}_{1}(1, q)+B_{0}\right) \int_{0}^{\infty} f v .
\end{aligned}
$$

Теперь рассмотрим случай $0<q<p<1$. Замена $f^{p} \rightarrow f$ в $(2.22)$ приводит к эквивалентному неравенству

$$
\left(\int_{0}^{\infty}\left(\int_{0}^{x} f^{1 / p} u\right)^{q} w(x) d x\right)^{p / q} \leqslant C^{p} \int_{0}^{\infty} f v, \quad f \in \mathfrak{M}^{\downarrow}
$$

Рассуждая аналогично предыдущему абзацу и применяя неравенство Минковского, получим

$$
\begin{aligned}
\left(\int_{0}^{\infty}\left(\int_{0}^{x} f^{1 / p} u\right)^{q} w(x) d x\right)^{p / q} & \lim _{n \rightarrow \infty}\left(\int_{0}^{\infty}\left(\int_{0}^{x}\left(\int_{y}^{\infty} h_{n}\right)^{1 / p} u(y) d y\right)^{q} w(x) d x\right)^{p / q} \\
\ll & \lim _{n \rightarrow \infty}\left(\int_{0}^{\infty}\left(\int_{0}^{x}\left(\int_{y}^{x} h_{n}\right)^{1 / p} u(y) d y\right)^{q} w(x) d x\right)^{p / q} \\
& +\lim _{n \rightarrow \infty}\left(\int_{0}^{\infty}\left(\int_{x}^{\infty} h_{n}\right)^{q / p} U^{q}(x) w(x) d x\right)^{p / q} \\
\ll & \lim _{n \rightarrow \infty}\left(\int_{0}^{\infty}\left(\int_{0}^{x} h_{n} U^{p}\right)^{q / p} w(x) d x\right)^{p / q} \\
& +B_{0}\left(1, \frac{q}{p}\right) \lim _{n \rightarrow \infty}^{\infty}\left(\int_{0}^{\infty} h_{n}\right) v(x) d x \\
\ll & \left(\mathscr{B}_{1}\left(1, \frac{q}{p}\right)+B_{0}\left(1, \frac{q}{p}\right)\right)_{0}^{\infty} f v=\left(\mathscr{B}_{1}^{p}(p, q)+B_{0}^{p}(p, q)\right) \int_{0}^{\infty} f v,
\end{aligned}
$$

и оценка сверху $C \ll B_{0}+\mathscr{B}_{1}$ доказана. Остается доказать оценку снизу $C \gg \mathscr{B}_{1}$, так как $C \gg B_{0}$ уже получена. Воспользуемся одной идеей из [2]. Обозначим $\mathscr{V}(t):=\operatorname{ess}_{\sup } \in[0, t]\left[U^{p}(s) / V(s)\right)$ и предположим сначала, что $\mathscr{V}(0)=0$. Заметим, что $\mathscr{V}(t)$ - непрерывная неубывающая функция. Положим

$$
g(t):=\max \left\{2^{m}, m \in \mathbb{Z}: 2^{m} \leqslant \mathscr{V}^{r / p}(t)\right\}, \quad \tau_{m}:=\inf \left\{y \in[0, \infty): 2^{m} \leqslant \mathscr{V}^{r / p}(y)\right\} .
$$

Так как $\mathscr{V}(t)$ непрерывна и не убывает, то величины $\tau_{m}$ существуют для всех $m \in \mathbb{Z}$. Очевидно, что они возрастают. Из их определений имеем

$$
\begin{gathered}
\frac{U^{r}\left(\tau_{m}\right)}{V^{r / p}\left(\tau_{m}\right)}=2^{m}=\mathscr{V}^{r / p}\left(\tau_{m}\right) \leqslant \mathscr{V}_{p}^{r / p}(t) \leqslant 2^{m+1}, \quad t \in\left[\tau_{m}, \tau_{m+1}\right], \\
g\left(\tau_{m}\right)=2^{m}, \quad g(s) \leqslant 2^{m-1} \quad \text { для всех } s \in\left[0, \tau_{m}\right) .
\end{gathered}
$$

Заметим, что

$$
g(t)=\sum_{m \in \mathbb{Z}} 2^{m} \chi_{\left[\tau_{m}, \tau_{m+1}\right)}(t) \leqslant \mathscr{V}^{r / p}(t)
$$


и определим

$$
f(t):=\int_{t}^{\infty} \frac{\left(\int_{x}^{\infty} w\right)^{r / q}}{V(x)} d g(x)
$$

где внешний интеграл понимается в смысле Стилтьеса. Тогда $f \in \mathfrak{M}^{\downarrow}$ и, используя (2.30), получаем

$$
\begin{aligned}
\int_{0}^{\infty} f v & =\int_{0}^{\infty}\left(\int_{x}^{\infty} w\right)^{r / q} d g(x)=\frac{q}{r} \int_{0}^{\infty} g(x)\left(\int_{x}^{\infty} w\right)^{r / p} w(x) d x \\
& \leqslant \frac{q}{r} \int_{0}^{\infty} \mathscr{V}^{r / p}(x)\left(\int_{x}^{\infty} w\right)^{r / p} w(x) d x=: \frac{q}{r} \mathscr{B}_{1}^{r} .
\end{aligned}
$$

С другой стороны,

$$
\begin{aligned}
&\left(\int_{0}^{\infty}\left(\int_{0}^{x} f^{1 / p}(y) d U(y)\right)^{q} w(x) d x\right)^{1 / q} \\
& \geqslant\left(\sum_{m} \int_{\tau_{m}}^{\tau_{m+1}} w(x)\left(\int_{0}^{\tau_{m}}\left(\int_{y}^{\tau_{m}} \frac{\left(\int_{s}^{\infty} w\right)^{r / q}}{V(s)} d g(s)\right)^{1 / p} d U(y)\right)^{q} d x\right)^{1 / q} \\
& \geqslant\left(\sum_{m}\left(\int_{\tau_{m}}^{\tau_{m+1}} w\right)\left(\int_{\tau_{m}}^{\infty} w\right)^{r / p}\right. \\
&\left.\times\left(V^{-1 / p}\left(\tau_{m}\right) \int_{0}^{\tau_{m}}\left(g\left(\tau_{m}\right)-g(y)\right)^{1 / p} d U(y)\right)^{q}\right)^{1 / q} \\
& \gg\left(\sum_{m}\left(\int_{\tau_{m}}^{\tau_{m+1}} w\right)\left(\int_{\tau_{m}}^{\infty} w\right)^{r / p}\left(\frac{2^{m / p} U\left(\tau_{m}\right)}{V\left(\tau_{m}\right)^{1 / p}}\right)^{q}\right)^{1 / q} \\
& \geqslant\left(\sum_{m} 2^{m} \int_{\tau_{m}}^{\tau_{m+1}}\left(\int_{s}^{\infty} w\right)^{r / p} w(s) d s\right)^{1 / q} \\
& \gg\left(\int_{0}^{\infty} \mathscr{V}^{r / p}(s)\left(\int_{s}^{\infty} w\right)^{r / p} w(s) d s\right)^{1 / q}=\mathscr{B}_{1}^{r / q} .
\end{aligned}
$$

Неравенство (2.29) с функцией $f$, определенной в $(2.31)$, влечет $C^{p} \mathscr{B}_{1}^{r} \gg \mathscr{B}_{1}^{p r / q}$. Следовательно, $C \gg \mathscr{B}_{1}$. Теперь покажем, как избавиться от ограничения $\mathscr{V}(0)=0$. Пусть $\varepsilon>0$ - малое число. Положим $u_{\varepsilon}(x):=u(x) \chi_{[\varepsilon, \infty]}(x)$. Тогда неравенство (2.29) будет выполняться с $u_{\varepsilon}$ вместо $u$ с той же константой $C$. Если положить $U_{\varepsilon}(t)=\int_{0}^{t} u_{\varepsilon}, \mathscr{V}_{\varepsilon}:=\operatorname{ess}_{\sup _{s \in[0, t]}}\left(U_{\varepsilon}^{p}(s) / V(s)\right)$, то очевидно, что $\mathscr{V}_{\varepsilon}(0)=0$. Пусть

$$
\mathscr{B}_{1, \varepsilon}:=\left(\int_{0}^{\infty}\left(\operatorname{essup}_{s \in[0, t]} \frac{U_{\varepsilon}^{p}(s)}{V(s)}\right)^{r / p}\left(\int_{t}^{\infty} w\right)^{r / p} w(t) d t\right)^{1 / r} .
$$

Тогда из предыдущего рассуждения следует, что $\mathscr{B}_{1, \varepsilon} \ll C$. Отсюда по теореме о монотонной сходимости вытекает оценка $\mathscr{B}_{1} \ll C$. Теорема 2.5 доказана. 


\section{3. Редукционные теоремы}

В этом разделе мы предлагаем новый метод редукции $\left(L_{p, v}^{\downarrow} \rightarrow L_{q, w}^{+}\right)$- и $\left(L_{p, v}^{\uparrow} \rightarrow L_{q, w}^{+}\right)$-неравенств для положительных монотонны ности, мы существенно дополняем некоторые результаты из [30].

Далее мы будем использовать следующие условия:

(i) $T(\lambda f)=\lambda T f$ для всех $\lambda \geqslant 0$ и $f \in \mathfrak{M}^{+}$;

(ii) $T f(x) \leqslant c T g(x)$ для почти всех $x \in \mathbb{R}_{+}$, если $f(x) \leqslant g(x)$ для почти всех $x \in \mathbb{R}_{+}$с константой $c>0$, не зависящей от $f$ и $g$;

(iii) $T(f+\lambda \mathbf{1}) \leqslant c(T f+\lambda T \mathbf{1})$ для всех $f \in \mathfrak{M}^{+}$и $\lambda \geqslant 0$ с константой $c>0$, независящей от $f$ и $\lambda$, где $\mathbf{1}$ - функция на $\mathbb{R}_{+}$, тождественно равная 1 .

В п. 3.1 мы получаем редукционные теоремы для неравенства

$$
\left(\int_{0}^{\infty}(T f(t))^{q} w(t) d t\right)^{1 / q} \leqslant C\left(\int_{0}^{\infty}(f(t))^{p} v(t) d t\right)^{1 / p}
$$

на конусах $\mathfrak{M}^{\downarrow}$ и $\mathfrak{M}^{\uparrow}$ при $0<q<\infty, 1 \leqslant p<\infty$ (см. теоремы 3.1-3.4). В отличие от редукционных теорем, основанных на принципе двойственности Сойера, полученная область изменения параметров суммирования позволяет исследовать неравенства при $0<q<1 \leqslant p<\infty$. В п. 3.2 мы исследуем случай $0<p \leqslant q<\infty$ при некоторых дополнительных ограничениях на монотонный оператор.

В оставшемся наиболее трудном случае $0<q<p \leqslant 1$ мы не даем общей теории. Однако в пп. 3.3, 3.4 мы приводим полную характеризацию неравенств

$$
\left(\int_{0}^{\infty}\left(\int_{x}^{\infty} k(t, x) f(t) u(t) d t\right)^{q} w(x) d x\right)^{1 / q} \leqslant C\left(\int_{0}^{\infty}(f(t))^{p} v(t) d t\right)^{1 / p}
$$

и

$$
\left(\int_{0}^{\infty}\left(\int_{0}^{x} k(x, t) f(t) u(t) d t\right)^{q} w(x) d x\right)^{1 / q} \leqslant C\left(\int_{0}^{\infty}(f(t))^{p} v(t) d t\right)^{1 / p}
$$

с ядрами Ойнарова на конусах $\mathfrak{M}^{\downarrow}$ и $\mathfrak{M}^{\uparrow}$.

3.1. Редукционные теоремы для монотонных операторов при $0<$ $q \leqslant \infty, 1 \leqslant p<\infty$. Обозначим $V(t):=\int_{0}^{t} v$.

TеОрема 3.1. Пусть $0<q \leqslant \infty, 1<p<\infty u T: \mathfrak{M}^{+} \rightarrow \mathfrak{M}^{+}-$положительный оператор. Тогда из условия (3.1) следует неравенство

$$
\left(\int_{0}^{\infty}\left(T\left(\int_{x}^{\infty} h\right)\right)^{q} w\right)^{1 / q} \leqslant C\left(\int_{0}^{\infty} h^{p} V^{p} v^{1-p}\right)^{1 / p}, \quad h \in \mathfrak{M}^{+} .
$$

Если $V(\infty)=\infty$ и $T$ - монотонный оператор, удовлетворяющий условиям (i) и (ii), то условие (3.4) является достаточным для выполнения (3.1) на конусе $\mathfrak{M}^{\downarrow}$, а если $0<V(\infty)<\infty$, то для выполнения (3.1) на конусе $\mathfrak{M}^{\downarrow}$ достаточно (3.4) и

$$
\left(\int_{0}^{\infty}(T \mathbf{1})^{q} w\right)^{1 / q} \leqslant C\left(\int_{0}^{\infty} v\right)^{1 / p}
$$

если оператор Т удовлетворяет условиям (i)-(iii). 
ДоКАЗАТЕЛЬСТво. Пусть $0<q<\infty$.

Необходимость. Пусть функция $h \in \mathfrak{M}^{+}$суммируема на $[x, \infty)$ для любого $x>0$. Тогда $f(x)=\int_{x}^{\infty} h \in \mathfrak{M}^{\downarrow}$, и по неравенству (3.1), неравенству Харди (2.10) при $p>1$ и по теореме Фубини при $p=1$ находим

$$
\begin{aligned}
\left(\int_{0}^{\infty}\left(T\left(\int_{x}^{\infty} h\right)\right)^{q} w\right)^{1 / q} & \leqslant C\left(\int_{0}^{\infty}\left(\int_{x}^{\infty} h\right)^{p} v(x) d x\right)^{1 / p} \\
& \ll C\left(\int_{0}^{\infty} h^{p} V^{p} v^{1-p}\right)^{1 / p},
\end{aligned}
$$

откуда следует (3.4).

Достаточность. Пусть сначала $V(\infty)=\infty$ и $f \in \mathfrak{M}^{\downarrow}$. Тогда

$$
\begin{aligned}
f(x) & =\frac{f(x) V^{2}(x)}{V^{2}(x)}=2\left(\int_{x}^{\infty} \frac{v}{V^{3}}\right) f(x) V^{2}(x) \\
& \leqslant\left(\int_{x}^{\infty} \frac{v}{V^{3}}\right) \int_{0}^{x} f V v \leqslant \int_{x}^{\infty}\left(\int_{0}^{t} f V v\right) \frac{v(t) d t}{V^{3}(t)} .
\end{aligned}
$$

Применяя (ii) и (3.4) с функцией

$$
h(t)=\left(\int_{0}^{t} f V v\right) \frac{v(t)}{V^{3}(t)},
$$

по неравенству Харди (2.10) при $p>1$ и по теореме Фубини при $p=1$ находим

Если $0<V(\infty)<\infty$, то

$$
\begin{aligned}
\left(\int_{0}^{\infty}(T f)^{q} w\right)^{1 / q} & \leqslant C\left(\int_{0}^{\infty}\left(\int_{0}^{t} f V v\right)^{p} \frac{v(t) d t}{V^{2 p}(t)}\right)^{1 / p} \\
& \ll C\left(\int_{0}^{\infty} f^{p} v\right)^{1 / p} .
\end{aligned}
$$

$$
\begin{aligned}
f(x) & =\left[\frac{1}{V^{2}(x)}-\frac{1}{V^{2}(\infty)}\right] f(x) V^{2}(x)+\frac{V^{2}(x)}{V^{2}(\infty)} f(x) \\
& \leqslant 4\left(\int_{x}^{\infty} \frac{v}{V^{3}}\right) \int_{0}^{x} f V v+\frac{V^{1 / p^{\prime}}(x) V^{1 / p}(x)}{V(\infty)} f(x) \\
& \leqslant 4 \int_{x}^{\infty}\left(\int_{0}^{t} f V v\right) \frac{v(t) d t}{V^{3}(t)}+\frac{\left(\int_{0}^{\infty} f^{p} v\right)^{1 / p}}{V^{1 / p}(\infty)}=: \int_{x}^{\infty} h+\lambda \mathbf{1} .
\end{aligned}
$$

Далее, применяя (i)-(iii), (3.4), (3.5), неравенство Харди при $p>1$ и теорему Фубини при $p=1$, получаем

$$
\begin{aligned}
\left(\int_{0}^{\infty}(T f)^{q} w\right)^{1 / q} & \ll\left(\int_{0}^{\infty}\left(T\left(\int_{x}^{\infty} h\right)\right)^{q} w\right)^{1 / q}+\lambda\left(\int_{0}^{\infty}(T \mathbf{1})^{q} w\right)^{1 / q} \\
& \ll C\left(\left(\int_{0}^{\infty}\left(\int_{0}^{t} f V v\right)^{p} \frac{v(t) d t}{V^{2 p}(t)}\right)^{1 / p}+\left(\int_{0}^{\infty} f^{p} v\right)^{1 / p}\right) \\
& \ll C\left(\int_{0}^{\infty} f^{p} v\right)^{1 / p} .
\end{aligned}
$$

Случай $q=\infty$ доказывается аналогично. Теорема 3.1 доказана. 
Полезным альтернативным утверждением является следующая редукционная теорема.

Tеорема 3.2. Пусть $0<q \leqslant \infty, 1 \leqslant p<\infty$ u onepamop $T: \mathfrak{M}^{+} \rightarrow \mathfrak{M}^{+}$ удовлетворяет условиям (i) u (ii). Тогда для выполнения на конусе $\mathfrak{M}^{\downarrow}$ неравенства (3.1) достаточно, а при $V(\infty)=\infty$ необходимо, чтобъ

$$
\left(\int_{0}^{\infty}\left(T\left(\frac{1}{V^{2}(x)} \int_{0}^{x} h V\right)\right)^{q} w\right)^{1 / q} \leqslant C\left(\int_{0}^{\infty} h^{p} v^{1-p}\right)^{1 / p}, \quad h \in \mathfrak{M}^{+} .
$$

Если $0<V(\infty)<\infty$, то (3.1) необходимо для (3.6) при выполнении условий (i)-(iii) и неравенства (3.5).

ДокАЗАТЕЛЬСтво. Пусть $0<q<\infty$.

Достаточность. Если $f \in \mathfrak{M}^{\downarrow}$, то $f(x) \leqslant \frac{2}{V^{2}(x)} \int_{0}^{x} f v V$. Применяя (ii) и $(3.6)$ с $h=2 f v$, получим

$$
\begin{aligned}
\left(\int_{0}^{\infty}(T f)^{q} w\right)^{1 / q} & \ll\left(\int_{0}^{\infty}\left(T\left(\frac{1}{V^{2}(x)} \int_{0}^{x} h V\right)\right)^{q} w(x) d x\right)^{1 / q} \\
& \leqslant C\left(\int_{0}^{\infty} h^{p} v^{1-p}\right)^{1 / p}=2 C\left(\int_{0}^{\infty} f^{p} v\right)^{1 / p}
\end{aligned}
$$

Необходимость. Пусть сначала $V(\infty)=\infty$ и функция $h \in \mathfrak{M}^{+}$суммируема на $[0, x]$ для всех $x>0$. Тогда

$$
\frac{1}{V^{2}(x)} \int_{0}^{x} h V=2 \int_{x}^{\infty} \frac{v}{V^{3}} \int_{0}^{x} h V \leqslant 2 \int_{x}^{\infty} \frac{v(s) d s}{V^{3}(s)} \int_{0}^{s} h V=: f(x) \in \mathfrak{M}^{\downarrow} .
$$

По условию (ii) и неравенству (3.1) находим

$$
\begin{aligned}
J & :=\left(\int_{0}^{\infty}\left(T\left(\frac{1}{V^{2}(x)} \int_{0}^{x} h V\right)\right)^{q} w\right)^{1 / q} \ll\left(\int_{0}^{\infty}(T f)^{q} w\right)^{1 / q} \leqslant C\left(\int_{0}^{\infty} f^{p} v\right)^{1 / p} \\
& =2 C\left(\int_{0}^{\infty}\left(\int_{x}^{\infty} \frac{v(s) d s}{V^{3}(s)} \int_{0}^{s} h V\right)^{p} v(x) d x\right)^{1 / p} .
\end{aligned}
$$

Если $p=1$, то

$$
J \ll C \int_{0}^{\infty}\left(\int_{x}^{\infty} \frac{v(s) d s}{V^{3}(s)} \int_{0}^{s} h V\right) v(x) d x=C \int_{0}^{\infty} \frac{v(s) d s}{V^{2}(s)} \int_{0}^{s} h V=C \int_{0}^{\infty} h .
$$

Пусть $p>1$. Имеем

$$
2 \int_{x}^{\infty} \frac{v(s) d s}{V^{3}(s)} \int_{0}^{s} h V=\frac{1}{V^{2}(x)} \int_{0}^{x} h V+\int_{x}^{\infty} \frac{h}{V}
$$

и, применяя неравенства Харди, получаем (3.6). 
Далее, если $V(\infty) \in(0, \infty)$, то запишем

$$
\begin{aligned}
\frac{1}{V^{2}(x)} \int_{0}^{x} h V & =\left[\frac{1}{V^{2}(x)}-\frac{1}{V^{2}(\infty)}\right] \int_{0}^{x} h V+\frac{\int_{0}^{x} h V}{V^{2}(\infty)} \\
& \leqslant 2 \int_{x}^{\infty} \frac{v(s) d s}{V^{3}(s)} \int_{0}^{s} h V+\frac{\int_{0}^{\infty} h V}{V^{2}(\infty)}=: f_{1}(x)+\lambda \mathbf{1} .
\end{aligned}
$$

Применим свойство (iii). Предыдущие рассуждения дают нам оценку первого слагаемого:

$$
\left(\int_{0}^{\infty}\left(T f_{1}\right)^{q} w\right)^{1 / q} \ll C\left(\int_{0}^{\infty} h^{p} v^{1-p}\right)^{1 / p}
$$

Для оценки второго слагаемого используем свойство (i), (3.5) и неравенство Гёльдера:

$$
\begin{aligned}
\left(\int_{0}^{\infty}(T(\lambda \mathbf{1}))^{q} w\right)^{1 / q} & \leqslant C \lambda\left(\int_{0}^{\infty} v\right)^{1 / p}=C \frac{V^{1 / p}(\infty) \int_{0}^{\infty} h V}{V^{2}(\infty)} \\
& \leqslant C\left(\int_{0}^{\infty} h^{p} v^{1-p}\right)^{1 / p}
\end{aligned}
$$

откуда следует необходимость (3.6).

Случай $q=\infty$ доказывается аналогично. Теорема 3.2 доказана.

Рассмотрим неравенство

$$
\left(\int_{0}^{\infty}(T f(t))^{q} w(t) d t\right)^{1 / q} \leqslant C\left(\int_{0}^{\infty}(f(t))^{p} v(t) d t\right)^{1 / p}, \quad f \in \mathfrak{M}^{\uparrow} .
$$

Положим $V_{*}(t):=\int_{t}^{\infty} v$.

TеOрема 3.3. Пусть $0<q \leqslant \infty, 1 \leqslant p<\infty u T: \mathfrak{M}^{+} \rightarrow \mathfrak{M}^{+}-$положительный оператор. Тогда из условия (3.7) следует неравенство

$$
\left(\int_{0}^{\infty}\left(T\left(\int_{0}^{x} h\right)\right)^{q} w\right)^{1 / q} \leqslant C\left(\int_{0}^{\infty} h^{p} V_{*}^{p} v^{1-p}\right)^{1 / p}, \quad h \in \mathfrak{M}^{+} .
$$

Если $V(\infty)=\infty$ и $T$ - монотонный оператор, удовлетворяющий условиям (i) u (ii), то (3.8) достаточно для выполнения (3.7). Если $0<V(\infty)<\infty u T-$ монотонный оператор, удовлетворяющий условиям (i)-(iii), то для для (3.7) достаточно (3.8) и (3.5).

Tеорема 3.4. Пусть $0<q<\infty, 1 \leqslant p<\infty$ и onepamop $T: \mathfrak{M}^{+} \rightarrow \mathfrak{M}^{+}$ подчиняется условиям (i) $и$ (ii). Тогда для выполнения (3.7) достаточно, а при $V(\infty)=\infty$ необходимо, чтобъ

$$
\left(\int_{0}^{\infty}\left(T\left(\frac{1}{V_{*}^{2}(x)} \int_{x}^{\infty} h V_{*}\right)\right)^{q} w\right)^{1 / q} \leqslant C\left(\int_{0}^{\infty} h^{p} v^{1-p}\right)^{1 / p}, \quad h \in \mathfrak{M}^{+} .
$$

Если $0<V(\infty)<\infty$, то (3.7) необходимо для (3.9) при выполнении условий (i)-(iii) $и$ (3.5). 
3.2. Редукционные теоремы для квазилинейных операторов при $0<p \leqslant q<\infty$. Пусть $f \in \mathfrak{M}^{\downarrow}$. Тогда существует последовательность $\left\{x_{n}\right\} \subset$ $\mathbb{R}_{+}$такая, что

$$
\begin{aligned}
f(x) & \approx \sum_{n} 2^{-n} \chi_{\left[0, x_{n}\right]}(x)=\sum_{n: x_{n} \geqslant x} 2^{-n} \chi_{\left[0, x_{n}\right]}(x) \\
& =\int_{[x, \infty)}\left(\sum_{n} 2^{-n} \delta_{x_{n}}(s)\right) d s=: \int_{[x, \infty)} h(s) d s,
\end{aligned}
$$

где $\delta_{t}(s)$ - дельта-функция Дирака в точке $t$. Заметим, что

$$
[f(x)]^{r} \approx\left(\sum_{n} 2^{-n} \chi_{\left[0, x_{n}\right]}(x)\right)^{r} \approx \sum_{n} 2^{-n r} \chi_{\left[0, x_{n}\right]}(x), \quad r>0 .
$$

Теорема 3.5. Пусть $0<p \leqslant q<\infty$ u для оператора $T: \mathfrak{M}^{+} \rightarrow \mathfrak{M}^{+}$выполнены условия (i)-(iii), а также условие

$$
T\left(\sum_{n} f_{n}\right) \ll\left(\sum_{n}\left[T f_{n}\right]^{p}\right)^{1 / p}
$$

для любых $f_{n} \geqslant 0$. Тогда (3.1) на конусе $\mathfrak{M}^{\downarrow}$ эквивалентно выполнению любого из следующих условий:

$$
\begin{aligned}
& \left(\int_{0}^{\infty}\left(\int_{0}^{\infty}\left[T \chi_{[0, s]}(x)\right]^{p} h(s) d s\right)^{q / p} w(x) d x\right)^{p / q} \leqslant C_{2}^{p} \int_{0}^{\infty} h V, \quad h \in \mathfrak{M}^{+}, \\
& \left(\int_{0}^{\infty}\left[\sup _{s>0} T \chi_{[0, s]}(x) f(s)\right]^{q} w(x) d x\right)^{1 / q} \leqslant C_{3}\left(\int_{0}^{\infty} f^{p} v\right)^{1 / p}, \quad f \in \mathfrak{M}^{\downarrow}, \\
& \left(\int_{0}^{\infty}\left[\sup _{s>0}\left(T \chi_{[0, s]}(x)\right)^{p} \int_{s}^{\infty} h\right]^{q / p} w(x) d x\right)^{p / q} \leqslant C_{4}^{p} \int_{0}^{\infty} h V, \quad h \in \mathfrak{M}^{+},
\end{aligned}
$$

или

$$
\mathbf{D}:=\sup _{t>0}\left(\int_{0}^{\infty}\left[T \chi_{[0, t]}(x)\right]^{q} w(x) d x\right)^{1 / q} V^{-1 / p}(t)<\infty .
$$

Более того,

$$
C \approx C_{2}=\mathbf{D} \approx C_{3}=C_{4} .
$$

ДоказАтельство. Эквивалентность $(3.14) \Leftrightarrow(3.15)$ с равенством $C_{3}=C_{4}$ доказываем, применяя предложение 2.1. (3.14) $\Rightarrow$ (3.16) получается применением $(3.14)$ к функции $f_{t}(s):=\chi_{[0, t]}(s), t>0$. Аналогично доказываем импликацию $(3.1) \Rightarrow(3.16)$. Из свойств (i)-(iii) находим, что для всех $s>0$

$$
T f(x) \geqslant T\left(\chi_{[0, s]} f\right)(x) \geqslant T \chi_{[0, s]}(x) f(s),
$$

и импликация $(3.1) \Rightarrow(3.14)$ доказана. Пусть

$$
k(x, s):=\left[T \chi_{[0, s]}(x)\right]^{p} \quad \text { и } \quad \mathbf{K} h(x):=\int_{0}^{\infty} k(x, s) h(s) d s .
$$


Тогда (3.13) эквивалентно ограниченности $\mathbf{K}: L_{V}^{1} \rightarrow L_{w}^{q / p}$, и по теореме 1.1

$$
C_{2}^{p}=\|\mathbf{K}\|_{L_{V}^{1} \rightarrow L_{w}^{q / p}}=\mathbf{D}^{p} .
$$

Покажем теперь, что $(3.13) \Rightarrow(3.1)$. Из (3.11) и (3.12) следует, что

$$
\begin{aligned}
\left(T f^{1 / p}\right)(x) & \approx\left(T\left(\sum_{n} 2^{-n} \chi_{\left[0, x_{n}\right]}\right)^{1 / p}\right)(x) \\
& \approx T\left(\sum_{n} 2^{-n / p} \chi_{\left[0, x_{n}\right]}\right)(x) \ll\left(\sum_{n} 2^{-n}\left[T \chi_{\left[0, x_{n}\right]}(x)\right]^{p}\right)^{1 / p} .
\end{aligned}
$$

Заметим, что (3.1) эквивалентно неравенству

$$
\left(\int_{0}^{\infty}\left(T f^{1 / p}\right)^{q} w\right)^{p / q} \leqslant C^{p} \int_{0}^{\infty} f v, \quad f \in \mathfrak{M}^{\downarrow} .
$$

Пользуясь (3.11), находим

$$
\begin{aligned}
& \left(\int_{0}^{\infty}\left(T f^{1 / p}\right)^{q} w\right)^{p / q} \ll\left(\int_{0}^{\infty}\left(\sum_{n} 2^{-n}\left[T \chi_{\left[0, x_{n}\right]}(x)\right]^{p}\right)^{q / p} w(x) d x\right)^{p / q} \\
& \quad=\left(\int_{0}^{\infty}\left(\int_{0}^{\infty}\left[T \chi_{[0, s]}(x)\right]^{p} h(s) d s\right)^{q / p} w(x) d x\right)^{p / q} \\
& \quad \leqslant C_{2}^{p} \int_{0}^{\infty} h V=C_{2}^{p} \sum_{n} 2^{-n} V\left(x_{n}\right) \approx C_{2}^{p} \sum_{n} 2^{-n} \int_{\left(0, x_{n}\right]} v \approx C_{2}^{p} \int_{0}^{\infty} f v
\end{aligned}
$$

Следовательно, $C \ll C_{2}$, и (3.17) доказано. Теорема 3.5 доказана.

Аналогичный результат справедлив для конуса неубывающих функций.

Теорема 3.6. Пусть $0<p \leqslant q<\infty$ и для оператора $T: \mathfrak{M}^{+} \rightarrow \mathfrak{M}^{+}$выполнены условия (i)-(iii) и (3.12). Тогда неравенство (3.7) эквивалентно любому из следующих условий:

$$
\begin{aligned}
& \left(\int_{0}^{\infty}\left(\int_{0}^{\infty}\left[T \chi_{[s, \infty)}(x)\right]^{p} h(s) d s\right)^{q / p} w(x) d x\right)^{p / q} \leqslant C_{2}^{p} \int_{0}^{\infty} h V_{*}, \quad h \in \mathfrak{M}^{+}, \\
& \left(\int_{0}^{\infty}\left[\sup _{s>0} T \chi_{[s, \infty)}(x) f(s)\right]^{q} w(x) d x\right)^{1 / q} \leqslant C_{3}\left(\int_{0}^{\infty} f^{p} v\right)^{1 / p}, \quad f \in \mathfrak{M}^{\uparrow}, \\
& \left(\int_{0}^{\infty}\left[\sup _{s>0}\left(T \chi_{[s, \infty)}(x)\right)^{p} \int_{0}^{s} h\right]^{q / p} w(x) d x\right)^{p / q} \leqslant C_{4}^{p} \int_{0}^{\infty} h V, \quad h \in \mathfrak{M}^{+},
\end{aligned}
$$

или

$$
\mathbf{D}_{*}:=\sup _{t>0}\left(\int_{0}^{\infty}\left[T \chi_{[t, \infty)}(x)\right]^{q} w(x) d x\right)^{1 / q} V_{*}^{-1 / p}(t)<\infty .
$$

При этом

$$
C \approx C_{2}=\mathbf{D}_{*} \approx C_{3}=C_{4}
$$


Далее мы рассмотрим обратное неравенство

$$
\left(\int_{0}^{\infty} f^{q} w\right)^{1 / q} \leqslant C\left(\int_{0}^{\infty}(T f)^{p} v\right)^{1 / p}, \quad f \in \mathfrak{M}^{\downarrow} .
$$

Положим $W_{*}(t):=\int_{t}^{\infty} w$.

Tеорема 3.7. Пусть $0<p \leqslant q<\infty$ и оператор $T: \mathfrak{M}^{+} \rightarrow \mathfrak{M}^{+}$подчинен условиям (i)-(iіi), а такюе условию

$$
\left(\sum_{n}\left[T f_{n}\right]^{q}\right)^{1 / q} \ll T\left(\sum_{n} f_{n}\right)
$$

для любых $f_{n} \geqslant 0$. Тогда неравенство (3.23) эквивалентно выполнению любого из условий

$$
\int_{0}^{\infty} h W \leqslant C_{2}^{q}\left(\int_{0}^{\infty}\left(\int_{0}^{\infty}\left[T \chi_{[0, s]}(x)\right]^{p} h(s) d s\right)^{p / q} v(x) d x\right)^{q / p}, \quad h \in \mathfrak{M}^{+},
$$

или

$$
\mathfrak{D}:=\sup _{t>0} W^{1 / q}(t)\left(\int_{0}^{\infty}\left[T \chi_{[0, t]}(x)\right]^{p} v(x) d x\right)^{-1 / p}<\infty .
$$

Более того,

$$
C \approx C_{2}=\mathfrak{D} .
$$

ДокАЗАТЕЛЬство. Импликация $(3.23) \Rightarrow(3.26)$ очевидна. Покажем, что $(3.26) \Rightarrow(3.25)$. Применяя неравенство Минковского, имеем

$$
\begin{aligned}
\int_{0}^{\infty} h W & \leqslant \mathfrak{D}^{q} \int_{0}^{\infty}\left(\int_{0}^{\infty}\left[T \chi_{[0, t]}(x)\right]^{p} v(x) d x\right)^{q / p} h(t) d t \\
& \leqslant \mathfrak{D}^{q}\left(\int_{0}^{\infty} v(x)\left(\int_{0}^{\infty}\left[T \chi_{[0, t]}(x)\right]^{q} h(t) d t\right)^{p / q} d x\right)^{q / p} .
\end{aligned}
$$

Далее, (3.23) эквивалентно неравенству

$$
\int_{0}^{\infty} f w \leqslant C\left(\int_{0}^{\infty}\left(T f^{1 / q}\right)^{p} v\right)^{q / p}, \quad f \in \mathfrak{M}^{\downarrow}
$$

Пользуясь $(3.25),(3.10),(3.11)$ и $(3.24)$, запишем

$$
\begin{aligned}
\int_{0}^{\infty} f w & \approx \int_{0}^{\infty} h W \leqslant C_{2}^{q}\left(\int_{0}^{\infty}\left(\int_{0}^{\infty}\left[T \chi_{[0, s]}(x)\right]^{q} h(s) d s\right)^{p / q} v(x) d x\right)^{q / p} \\
& =C_{2}^{q}\left(\int_{0}^{\infty}\left(\sum_{n} 2^{-n}\left[T \chi_{\left[0, x_{n}\right]}(x)\right]^{q}\right)^{p / q} v(x) d x\right)^{q / p} \\
& =C_{2}^{q}\left(\int_{0}^{\infty}\left(\sum_{n}\left[T\left(2^{-n / q} \chi_{\left[0, x_{n}\right]}\right)(x)\right]^{q}\right)^{p / q} v(x) d x\right)^{q / p} \\
& \leqslant C_{2}^{q}\left(\int_{0}^{\infty}\left[T\left(\sum_{n} 2^{-n / q} \chi_{\left[0, x_{n}\right]}\right)(x)\right]^{p} v(x) d x\right)^{q / p}
\end{aligned}
$$




$$
\begin{aligned}
& \approx C_{2}^{q}\left(\int_{0}^{\infty}\left[T\left(\sum_{n} 2^{-n} \chi_{\left[0, x_{n}\right]}\right)^{1 / q}(x)\right]^{p} v(x) d x\right)^{q / p} \\
& \approx C_{2}^{q}\left(\int_{0}^{\infty}\left(T f^{1 / q}\right)^{p} v\right)^{q / p}
\end{aligned}
$$

и (3.27) доказано. Теорема 3.7 доказана.

Аналогичным образом характеризуется неравенство

$$
\left(\int_{0}^{\infty} f^{q} w\right)^{1 / q} \leqslant C\left(\int_{0}^{\infty}(T f)^{p} v\right)^{1 / p}, \quad f \in \mathfrak{M}^{\uparrow}
$$

Tеорема 3.8. Для $0<p \leqslant q<\infty$ и оператора $T: \mathfrak{M}^{+} \rightarrow \mathfrak{M}^{+}$, для которого выполнены (i)-(iii) и (3.24), неравенство (3.29) эквивалентно любому из условий

$$
\int_{0}^{\infty} h W_{*} \leqslant C_{2}^{q}\left(\int_{0}^{\infty}\left(\left[T \chi_{[s, \infty)}(x)\right]^{p} h(s) d s\right)^{p / q} w(x) d x\right)^{q / p}, \quad h \in \mathfrak{M}^{+},
$$

или

$$
\mathfrak{D}_{*}:=\sup _{t>0} W_{*}^{1 / q}(t)\left(\int_{0}^{\infty}\left[T \chi_{[t, \infty)}(x)\right]^{p} v(x) d x\right)^{-1 / p}<\infty,
$$

при этом

$$
C \approx C_{2}=\mathfrak{D}_{*}
$$

ЗАмЕЧАНИЕ 3.1. Пусть $T$ - линейный интегральный оператор,

$$
T f(x):=\int_{0}^{\infty} k(x, y) f(y) d y
$$

с неотрицательным ядром. Тогда условие (3.12) выполняется для всех $p \in(0,1]$ и из наших теорем 3.5 и 3.6 вытекают теорема 4.1 из работы [97], теорема 2.1, (a) из работы [66]) и теорема 1 работы [6]. Аналогично, условие (3.24) верно для всех $q \geqslant 1$. Тогда по теоремам 3.7 и 3.8 мы получаем обобщение теоремы 4.2 из [97] и теоремы 2.1 , (b) из [66] на более широкий интервал параметров суммирования.

3.3. Редукционные теоремы для операторов Вольтерра при $0<q<$ $p \leqslant 1$. Пусть $u, v$ и $w$ - весовые функции. Напомним, что $U(t):=\int_{0}^{t} u$, $V(t):=\int_{0}^{t} v, W(t):=\int_{0}^{t} w$, и определим $U(y, x):=\int_{x}^{y} u$. Для простоты мы будем считать, что $0<U(t)<\infty, 0<V(t)<\infty, 0<W(t)<\infty$ для всех $t>0$ и $U(\infty)=V(\infty)=W(\infty)=\infty$. Обозначим

$$
K(x, y):=\int_{0}^{y} k(x, z) u(z) d z .
$$


ТеОрема 3.9. Пусть $0<q<p \leqslant 1$. Если $k(x, y) \geqslant 0$ - непрерывное ядро Ойнарова, то следующие условия эквивалентнъ:

$$
\begin{aligned}
& \left(\int_{0}^{\infty}\left(\int_{0}^{x} k(x, y) f(y) u(y) d y\right)^{q} w(x) d x\right)^{1 / q} \leqslant C_{1}\left(\int_{0}^{\infty} f^{p} v\right)^{1 / p}, \quad f \in \mathfrak{M}^{\downarrow}, \\
& \left(\int_{0}^{\infty}\left(\int_{0}^{x} K^{p}(x, y) h(y) d y+K^{p}(x, x) \int_{x}^{\infty} h(y) d y\right)^{q / p} w(x) d x\right)^{p / q} \\
& \leqslant C_{2}^{p} \int_{0}^{\infty} h V, h \in \mathfrak{M}^{+}, \\
& \left(\int_{0}^{\infty}\left(\sup _{0<y \leqslant x} K^{p}(x, y) \int_{y}^{\infty} h\right)^{q / p} w(x) d x\right)^{p / q} \leqslant C_{3}^{p} \int_{0}^{\infty} h V, \quad h \in \mathfrak{M}^{+}, \\
& \left(\int_{0}^{\infty}\left(\sup _{0<y \leqslant x} K(x, y) f(y)\right)^{q} w(x) d x\right)^{1 / q} \leqslant C_{4}\left(\int_{0}^{\infty} f^{p} v\right)^{1 / p}, \quad f \in \mathfrak{M}^{\downarrow}, \quad(3.37) \\
& \mathbb{B}^{r}:=\sup _{\left\{x_{k}\right\}} \sum_{k \in \mathbb{Z}}\left(\int_{x_{k}}^{x_{k+1}} K^{q}\left(y, x_{k}\right) w(y) d y\right)^{r / q} V^{-r / p}\left(x_{k}\right)<\infty .
\end{aligned}
$$

Более того,

$$
C_{1} \approx C_{2} \approx C_{3}=C_{4} \approx \mathbb{B} .
$$

Доказательство. Мы докажем, что $(3.35) \Rightarrow(3.34) \Rightarrow(3.37) \Leftrightarrow(3.36) \Rightarrow$ $(3.38) \Rightarrow(3.35)$. Неравенство (3.34) эквивалентно неравенству

$$
\left(\int_{0}^{\infty}\left(\int_{0}^{x} k(x, y) f^{1 / p}(y) u(y) d y\right)^{q} w(x) d x\right)^{p / q} \leqslant C_{1}^{p} \int_{0}^{\infty} f v, \quad f \in \mathfrak{M}^{\downarrow} .
$$

Пусть $f(x)=\int_{x}^{\infty} h$. Тогда по неравенству Минковского

$$
\begin{aligned}
\int_{0}^{x} k(x, z) f^{1 / p}(z) u(z) d z & =\int_{0}^{x}\left(\int_{z}^{\infty} h\right)^{1 / p} k(x, z) u(z) d z \\
& \leqslant\left(\int_{0}^{\infty}\left(\int_{0}^{x} \chi_{(z, \infty)}(y) k(x, z) u(z) d z\right)^{p} h(y) d y\right)^{1 / p} \\
& \approx\left(\int_{0}^{x} K^{p}(x, y) h(y) d y+K^{p}(x, x) \int_{x}^{\infty} h(y) d y\right)^{1 / p}
\end{aligned}
$$

и для доказательства $(3.35) \Rightarrow(3.34)$ остается применить предложение 2.1.

Для любой $f \in \mathfrak{M}^{\downarrow}$ имеем

$$
\int_{0}^{x} k(x, z) f(z) u(z) d z \geqslant \sup _{0<y \leqslant x} \int_{0}^{y} k(x, z) u(z) d z f(y) \geqslant \sup _{0<y \leqslant x} K(x, y) f(y) .
$$

Следовательно, $(3.34) \Rightarrow(3.37)$. Импликация $(3.37) \Rightarrow(3.36)$ очевидна, а импликацию $(3.36) \Rightarrow(3.37)$ доказываем, применяя предложение 2.1 и теорему Фату. 
Теперь допустим, что (3.36) выполняется и $\left\{x_{n}\right\} \subset \mathbb{R}_{+}-$произвольная возрастающая последовательность. Для любого $k \in \mathbb{Z}$ существует такая точка $\varepsilon_{k} \in\left(x_{k}, x_{k+1}\right)$, что $V\left(\varepsilon_{k}\right) \leqslant 2 V\left(x_{k}\right)$. Определим функцию

$$
h(x):=\sum_{k \in \mathbb{Z}} \frac{a_{k}}{x_{k}-\varepsilon_{k}} \chi_{\left(x_{k}, \varepsilon_{k}\right)}(x),
$$

где $\left\{a_{k}\right\} \subset \mathbb{R}_{+}$произвольны. Тогда, подставив эту функцию в неравенство (3.36), мы получим

$$
\left(\sum_{k \in \mathbb{Z}} a_{k}^{q / p} \int_{x_{k}}^{x_{k+1}} K^{q}\left(x, x_{k}\right) w(x) d x\right)^{p / q} \leqslant 2 C_{3}^{q} \sum_{k \in \mathbb{Z}} a_{k} V\left(x_{k}\right) .
$$

Отсюда вытекает $\mathbb{B} \ll C_{3}$.

Таким образом, остается только показать, что $(3.38) \Rightarrow(3.35)$. Используя условие Ойнарова (4), мы находим, что

$$
K(x, y) \approx k(x, y) \int_{0}^{y} u(z) d z+\int_{0}^{y} k(y, z) u(z) d z=k(x, y) U(y)+K(y, y),
$$

и отсюда следует, что (3.35) эквивалентно выполнению следующих трех неравенств:

$$
\begin{aligned}
& \left(\int_{0}^{\infty}\left(\int_{0}^{x} k(x, y)^{p} U(y)^{p} h(y) d y\right)^{q / p} w(x) d x\right)^{p / q} \leqslant C_{2}^{p} \int_{0}^{\infty} h V, \quad h \in \mathfrak{M}^{+}, \\
& \left(\int_{0}^{\infty}\left(\int_{0}^{x} K(y, y)^{p} h(y) d y\right)^{q / p} w(x) d x\right)^{p / q} \leqslant C_{2}^{p} \int_{0}^{\infty} h V, \quad h \in \mathfrak{M}^{+}, \quad \\
& \left(\int_{0}^{\infty}\left(\int_{x}^{\infty} h(y) d y\right)^{q / p} K(x, x)^{q} w(x) d x\right)^{p / q} \leqslant C_{2}^{p} \int_{0}^{\infty} h V, \quad h \in \mathfrak{M}^{+} .
\end{aligned}
$$

По теореме Лая [54; теорема 5] неравенство (3.41) выполняется тогда и только тогда, когда

$$
\begin{aligned}
& B_{1}^{r}:=\sup _{\left\{x_{k}\right\}} \sum_{k \in \mathbb{Z}}\left(\int_{x_{k}}^{x_{k+1}} k\left(y, x_{k}\right)^{q} w(y) d y\right)^{r / q} \sup _{y \in\left(x_{k-1}, x_{k}\right)} U(y)^{r} V^{-r / p}(y)<\infty, \\
& B_{2}^{r}:=\sup _{\left\{x_{k}\right\}} \sum_{k \in \mathbb{Z}}\left(\int_{x_{k}}^{x_{k+1}} w(y) d y\right)^{r / q} \sup _{y \in\left(x_{k-1}, x_{k}\right)} k^{r}\left(x_{k}, y\right) U(y)^{r} V^{-r / p}(y)<\infty,
\end{aligned}
$$

и неравенство (3.42) эквивалентно свойству

$$
B_{3}^{r}:=\sup _{\left\{x_{k}\right\}} \sum_{k \in \mathbb{Z}}\left(\int_{x_{k}}^{x_{k+1}} w(y) d y\right)^{r / q} \sup _{y \in\left(x_{k-1}, x_{k}\right)} K(y, y)^{r} V^{-r / p}(y)<\infty ;
$$

по теореме, двойственной к теореме 5 из [54], неравенство (3.43) эквивалентно

$$
B_{4}^{r}:=\sup _{\left\{x_{k}\right\}} \sum_{k \in \mathbb{Z}}\left(\int_{x_{k-1}}^{x_{k}} K(y, y)^{q} w(y) d y\right)^{r / q} V^{-r / p}\left(x_{k}\right)<\infty .
$$


Пусть $y_{k} \in\left(x_{k-1}, x_{k}\right)$ - такая точка, что

$$
\sup _{y \in\left(x_{k-1}, x_{k}\right)} U(y)^{r} V^{-r / p}(y)=U\left(y_{k}\right)^{r} V^{-r / p}\left(y_{k}\right) .
$$

Тогда

$$
\begin{aligned}
\sum_{k \in \mathbb{Z}}\left(\int_{x_{k}}^{x_{k+1}}\right. & \left.k\left(y, x_{k}\right)^{q} w(y) d y\right)^{r / q} \sup _{y \in\left(x_{k-1}, x_{k}\right)} U(y)^{r} V^{-r / p}(y) \\
\ll & \sum_{k \in \mathbb{Z}}\left(\int_{y_{k}}^{y_{k+2}} K\left(y, y_{k}\right)^{q} w(y) d y\right)^{r / q} V^{-r / p}\left(y_{k}\right) \\
\ll & \sum_{k \in \mathbb{Z}}\left(\int_{y_{2 k}}^{y_{2 k+2}} K\left(y, y_{2 k}\right)^{q} w(y) d y\right)^{r / q} V^{-r / p}\left(y_{2 k}\right) \\
+ & \sum_{k \in \mathbb{Z}}\left(\int_{y_{2 k+1}}^{y_{2 k+3}} K\left(y, y_{2 k+1}\right)^{q} w(y) d y\right)^{r / q} V^{-r / p}\left(y_{2 k+1}\right) \ll \mathbb{B}^{r} .
\end{aligned}
$$

Поэтому $B_{1} \ll \mathbb{B}$. Если $y_{k} \in\left(x_{k-1}, x_{k}\right)$ такова, что

$$
\sup _{y \in\left(x_{k-1}, x_{k}\right)} k^{r}\left(x_{k}, y\right) U(y)^{r} V^{-r / p}(y)=k^{r}\left(x_{k}, y_{k}\right) U\left(y_{k}\right)^{r} V^{-r / p}\left(y_{k}\right)
$$

то

$$
\begin{aligned}
\sum_{k \in \mathbb{Z}}\left(\int_{x_{k}}^{x_{k+1}} w(y) d y\right)^{r / q} \sup _{y \in\left(x_{k-1}, x_{k}\right)} k^{r}\left(x_{k}, y\right) U(y)^{r} V^{-r / p}(y) \\
\ll \sum_{k \in \mathbb{Z}}\left(\int_{y_{k}}^{y_{k+2}} K\left(y, y_{k}\right)^{q} w(y) d y\right)^{r / q} V^{-r / p}\left(y_{k}\right) \\
\ll \sum_{k \in \mathbb{Z}}\left(\int_{y_{2 k}}^{y_{2 k+2}} K\left(y, y_{2 k}\right)^{q} w(y) d y\right)^{r / q} V^{-r / p}\left(y_{2 k}\right) \\
+\sum_{k \in \mathbb{Z}}\left(\int_{y_{2 k+1}}^{y_{2 k+3}} K\left(y, y_{2 k+1}\right)^{q} w(y) d y\right)^{r / q} V^{-r / p}\left(y_{2 k+1}\right) \ll \mathbb{B}^{r} .
\end{aligned}
$$

Следовательно, $B_{2} \ll \mathbb{B}$. Для точки $y_{k} \in\left(x_{k-1}, x_{k}\right)$ такой, что

$$
\sup _{y \in\left(x_{k-1}, x_{k}\right)} K^{r}(y, y) V^{-r / p}(y)=K\left(y_{k}, y_{k}\right)^{r} V^{-r / p}\left(y_{k}\right),
$$

находим

$$
\begin{aligned}
\sum_{k \in \mathbb{Z}}\left(\int_{x_{k}}^{x_{k+1}} w(y) d y\right)^{r / q} \sup _{y \in\left(x_{k-1}, x_{k}\right)} K(y, y)^{r} V^{-r / p}(y) \\
\ll \sum_{k \in \mathbb{Z}}\left(\int_{y_{k}}^{y_{k+2}} K\left(y, y_{k}\right)^{q} w(y) d y\right)^{r / q} V^{-r / p}\left(y_{k}\right)
\end{aligned}
$$




$$
\begin{aligned}
& \ll \sum_{k \in \mathbb{Z}}\left(\int_{y_{2 k}}^{y_{2 k+2}} K\left(y, y_{2 k}\right)^{q} w(y) d y\right)^{r / q} V^{-r / p}\left(y_{2 k}\right) \\
& \quad+\sum_{k \in \mathbb{Z}}\left(\int_{y_{2 k+1}}^{y_{2 k+3}} K\left(y, y_{2 k+1}\right)^{q} w(y) d y\right)^{r / q} V^{-r / p}\left(y_{2 k+1}\right) \ll \mathbb{B}^{r},
\end{aligned}
$$

откуда $B_{3} \ll \mathbb{B}$. Так как $K(y, y) \ll K\left(y, x_{k-1}\right), y \in\left(x_{k-1}, x_{k}\right)$, то $B_{4} \ll \mathbb{B}$. Из полученных оценок сверху вытекает $C_{2} \ll \mathbb{B}$. Теорема 3.9 доказана.

Аналогичный результат для конуса неубывающих функций представлен в следующем утверждении.

Теорема 3.10. Пусть $0<q<p \leqslant 1$. Для непреръвного ядра Ойнарова $k(x, y)$ положим $K_{*}(y, x)=\int_{y}^{\infty} k(z, x) u(z) d z$. Тогда следующие неравенства
эквивалентны:

$$
\begin{gathered}
\left(\int_{0}^{\infty}\left(\int_{x}^{\infty} k(y, x) f(y) u(y) d y\right)^{q} w(x) d x\right)^{1 / q} \leqslant C_{1}\left(\int_{0}^{\infty} f^{p} v\right)^{1 / p}, \quad f \in \mathfrak{M}^{\uparrow}, \\
\left(\int_{0}^{\infty}\left(\int_{x}^{\infty} K_{*}^{p}(y, x) h(y) d y+K_{*}^{p}(x, x) \int_{0}^{x} h(y) d y\right)^{q / p} w(x) d x\right)^{p / q} \\
\leqslant C_{2}^{p} \int_{0}^{\infty} h V_{*}, \quad h \in \mathfrak{M}^{+}, \\
\left(\int_{0}^{\infty}\left(\sup _{y \geqslant x} K_{*}^{p}(y, x) \int_{0}^{y} h\right)^{q / p} w(x) d x\right)^{p / q} \leqslant C_{3}^{p} \int_{0}^{\infty} h V_{*}, \quad h \in \mathfrak{M}^{+}, \\
\left(\int_{0}^{\infty}\left(\sup _{y \geqslant x} K_{*}(y, x) f(y)\right)^{q} w(x) d x\right)^{1 / q} \leqslant C_{4}\left(\int_{0}^{\infty} f^{p} v\right)^{1 / p}, \quad f \in \mathfrak{M}^{\uparrow}, \\
\mathbb{B}_{*}^{r}:=\sup _{\left\{x_{k}\right\}} \sum_{k \in \mathbb{Z}}\left(\int_{x_{k-1}}^{x_{k}} K_{*}^{q}\left(x_{k}, y\right) w(y) d y\right)^{r / q} V_{*}^{-r / p}\left(x_{k}\right)<\infty
\end{gathered}
$$

$\left(\right.$ здесъ $\left.V_{*}(t):=\int_{t}^{\infty} v\right)$. Кроме того,

$$
C_{1} \approx C_{2} \approx C_{3}=C_{4} \approx \mathbb{B}_{*} .
$$

Для доказательства следующего результата нам потребуется утверждение о ядрах Ойнарова, представляющее самостоятельный интерес.

Лемма 3.1. Пусть измеримая функция $k(x, y) \geqslant 0$ на $\{(x, y): x \geqslant y \geqslant 0\}$ удовлетворяет правому неравенству в условии Ойнарова (4). Тогда существует $\alpha_{D} \in(0,1)$ такое, что для всех $\alpha \in\left(0, \alpha_{D}\right]$ и произвольной последовательности $x_{1} \geqslant x_{2} \geqslant \cdots \geqslant x_{n}$ выполняется неравенство

$$
\left[k\left(x_{1}, x_{n}\right)\right]^{\alpha} \leqslant 2 \sum_{i=1}^{n-1}\left[k\left(x_{i}, x_{i+1}\right]^{\alpha} .\right.
$$

ДокАЗАТЕЛЬСтво. Положим

$$
\alpha_{D}:=\frac{\log 2}{\log [D(1+2 D)]}
$$


и покажем, что (3.44) верно для $\alpha=\alpha_{D}$. Тогда, по неравенству Йенсена, (3.44) будет выполняться для всех $\alpha \in\left(0, \alpha_{D}\right]$. Рассуждаем по индукции. Из (4) и неравенства Йенсена следует, что

$$
k\left(x_{1}, x_{3}\right) \leqslant D\left(k\left(x_{1}, x_{2}\right)+k\left(x_{1}, x_{3}\right)\right) \leqslant D\left(k^{\alpha}\left(x_{1}, x_{2}\right)+k^{\alpha}\left(x_{1}, x_{3}\right)\right)^{1 / \alpha} .
$$

Следовательно,

$$
k^{\alpha}\left(x_{1}, x_{3}\right) \leqslant D^{\alpha}\left(k^{\alpha}\left(x_{1}, x_{2}\right)+k^{\alpha}\left(x_{1}, x_{3}\right)\right) .
$$

Так как $D^{\alpha} \leqslant 2$ при $\alpha=\alpha_{D}$, то (3.44) верно для $n=3$. Предположим, что (3.44) верно для всех $n=3, \ldots, k$ и

$$
a:=\sum_{i=1}^{k} k^{\alpha}\left(x_{i}, x_{i+1}\right)
$$

Тогда либо $k^{\alpha}\left(x_{1}, x_{2}\right) \leqslant a / 2$, либо $k^{\alpha}\left(x_{k}, x_{k+1}\right) \leqslant a / 2$. Без потери общности предположим, что $k^{\alpha}\left(x_{1}, x_{2}\right) \leqslant a / 2$. Пусть $j$ - наибольшее натуральное число такое, что

$$
\sum_{i=1}^{j} k^{\alpha}\left(x_{i}, x_{i+1}\right) \leqslant \frac{a}{2}
$$

Тогда $j<k$ и $\sum_{i=1}^{j+1} k^{\alpha}\left(x_{i}, x_{i+1}\right)>\frac{a}{2}$. Поэтому $\sum_{i=j+2}^{k} k^{\alpha}\left(x_{i}, x_{i+1}\right) \leqslant \frac{a}{2}$. По предположению индукции

$$
\begin{gathered}
k^{\alpha}\left(x_{1}, x_{j+1}\right) \leqslant 2 \sum_{i=1}^{j} k^{\alpha}\left(x_{i}, x_{i+1}\right) \leqslant a, \\
k^{\alpha}\left(x_{j+2}, x_{k+1}\right) \leqslant 2 \sum_{i=j+2}^{k} k^{\alpha}\left(x_{i}, x_{i+1}\right) \leqslant a
\end{gathered}
$$

и

$$
k^{\alpha}\left(x_{j+1}, x_{j+2}\right) \leqslant a .
$$

Следовательно,

$$
k\left(x_{1}, x_{j+1}\right) \leqslant a^{1 / \alpha}, \quad k\left(x_{j+2}, x_{k+1}\right) \leqslant a^{1 / \alpha}, \quad k\left(x_{j+1}, x_{j+2}\right) \leqslant a^{1 / \alpha} .
$$

Применяя (4), находим

$$
\begin{aligned}
k\left(x_{1}, x_{k+1}\right) & \leqslant D\left(k\left(x_{1}, x_{j+1}\right)+k\left(x_{j+1}, x_{k+1}\right)\right) \\
& \leqslant D\left(k\left(x_{1}, x_{j+1}\right)+D\left(k\left(x_{j+1}, x_{j+2}\right)+k\left(x_{j+2}, x_{k+1}\right)\right)\right) \\
& \leqslant D a^{1 / \alpha}+2 D^{2} a^{1 / \alpha}=D(1+2 D) a^{1 / \alpha} .
\end{aligned}
$$

Таким образом, если $\alpha=\alpha_{D}$, то

$$
k^{\alpha}\left(x_{1}, x_{k+1}\right) \leqslant D^{\alpha}(1+2 D)^{\alpha} a \leqslant 2 a
$$

и шаг индукции доказан. Лемма 3.1 доказана. 
В дальнейшем мы будем использовать известное соотношение (см. [35; предложение 2.1])

$$
\sum_{n} 2^{n}\left(\sum_{i=n}^{\infty} a_{i}\right)^{s} \approx \sum_{n} 2^{n} a_{n}^{s} s>0,
$$

верное для любой последовательности $\left\{a_{k}\right\} \subset \mathbb{R}_{+}$.

Положим

$$
U_{k}(y, x):=\int_{x}^{y} k(z, x) u(z) d z .
$$

Теорема 3.11. Пусть $0<q<p \leqslant 1 u$ ядро $k(x, y) \geqslant 0$ удовлетворяет условию Ойнарова (4). Тогда следующие утверждения эквивалентны:

$$
\begin{aligned}
& \left(\int_{0}^{\infty}\left(\int_{x}^{\infty} k(y, x) f(y) u(y) d y\right)^{q} w(x) d x\right)^{1 / q} \leqslant C_{1}\left(\int_{0}^{\infty} f^{p} v\right)^{1 / p}, \quad f \in \mathfrak{M}^{\downarrow}, \\
& \left(\int_{0}^{\infty}\left(\int_{x}^{\infty} U_{k}^{p}(y, x) h(y) d y\right)^{q / p} w(x) d x\right)^{p / q} \leqslant C_{2}^{p} \int_{0}^{\infty} h V, \quad h \in \mathfrak{M}^{+}, \quad \\
& \left(\int_{0}^{\infty}\left(\sup _{y \geqslant x} U_{k}(y, x) f(y)\right)^{q} w(x) d x\right)^{1 / q} \leqslant C_{3}\left(\int_{0}^{\infty} f^{p} v\right)^{1 / p}, \quad f \in \mathfrak{M}^{\downarrow}, \\
& \left(\int_{0}^{\infty}\left(\sup _{y \geqslant x} U_{k}^{p}(y, x) \int_{y}^{\infty} h\right)^{q / p} w(x) d x\right)^{p / q} \leqslant C_{4}^{p} \int_{0}^{\infty} h V, \quad h \in \mathfrak{M}^{+}, \\
& \mathbb{B}^{r}:=\sup _{\left\{x_{k}\right\}} \sum_{k \in \mathbb{Z}}\left(\int_{x_{k}}^{x_{k+1}} U_{k}^{q}\left(x_{k+1}, y\right) w(y) d y\right)^{r / q} V^{-r / p}\left(x_{k+1}\right)<\infty .
\end{aligned}
$$

При этом

$$
C_{1} \approx C_{2} \approx C_{3}=C_{4} \approx \mathbb{B} .
$$

ДокАЗАТЕЛьство. Мы покажем, что

$$
(3.50) \Rightarrow(3.47) \Rightarrow(3.46) \Rightarrow(3.48) \Leftrightarrow(3.49) \Rightarrow(3.50),
$$

и начнем с доказательства импликации $(3.50) \Rightarrow(3.47)$. Пусть $\left\{x_{n}\right\} \subset \mathbb{R}_{+}^{-}$ такая последовательность, что $2^{n}=\int_{0}^{x_{n}} w$; обозначим $\Delta_{n}:=\left[x_{n}, x_{n+1}\right)$. Тогда

$$
\begin{aligned}
J:= & \int_{0}^{\infty}\left(\int_{x}^{\infty} U_{k}^{p}(y, x) h(y) d y\right)^{q / p} w(x) d x \approx \sum_{n} 2^{n}\left(\int_{x_{n}}^{\infty} U_{k}^{p}\left(y, x_{n}\right) h(y) d y\right)^{q / p} \\
= & \sum_{n} 2^{n}\left(\sum_{i=n}^{\infty} \int_{\Delta_{i}} U_{k}^{p}\left(y, x_{n}\right) h(y) d y\right)^{q / p} \approx \sum_{n} 2^{n}\left(\sum_{i=n}^{\infty} \int_{\Delta_{i}} U_{k}^{p}\left(y, x_{i}\right) h(y) d y\right)^{q / p} \\
& +\sum_{n} 2^{n}\left(\sum_{i=n+1}^{\infty} k^{p}\left(x_{i}, x_{n}\right) \int_{\Delta_{i}}\left(\int_{x_{i}}^{y} u\right)^{p} h(y) d y\right)^{q / p} \\
& +\sum_{n} 2^{n}\left(\sum_{i=n+1}^{\infty} U_{k}^{p}\left(x_{i}, x_{n}\right) \int_{\Delta_{i}} h(y) d y\right)^{q / p} .
\end{aligned}
$$


Применяя (3.45) и лемму 3.1 с $\alpha=\min \left\{\alpha_{D}, p\right\}$, мы получим

$$
\begin{aligned}
J \ll \sum_{n} 2^{n}\left(\int_{\Delta_{n}} U_{k}^{p}\left(y, x_{n}\right) h(y) d y\right)^{q / p} & \\
& +\sum_{n} 2^{n}\left(\sum_{i=n+1}^{\infty}\left(\sum_{j=n}^{i-1} k^{\alpha}\left(x_{j+1}, x_{j}\right)\right)^{p / \alpha} \int_{\Delta_{i}}\left(\int_{x_{i}}^{y} u\right)^{p} h(y) d y\right)^{q / p} \\
& +\sum_{n} 2^{n}\left(\sum_{i=n+1}^{\infty}\left(\sum_{j=n}^{i-1} U_{k}\left(x_{j+1}, x_{j}\right)\right)^{p} \int_{\Delta_{i}} h(y) d y\right)^{q / p} \\
& +\sum_{n} 2^{n}\left(\sum_{i=n+2}^{\infty}\left(\sum_{j=n+1}^{i-1} k\left(x_{j}, x_{n}\right) \int_{\Delta_{j}} u\right)^{p} \int_{\Delta_{i}} h(y) d y\right)^{q / p} \\
=: \mathrm{I} & +\mathrm{II}+\mathrm{III}+\mathrm{IV} .
\end{aligned}
$$

Оценка суммы I. Имеем

$$
\begin{aligned}
& \int_{\Delta_{n}} U_{k}^{p}\left(y, x_{n}\right) h(y) d y=\int_{\Delta_{n}} U_{k}^{p}\left(y, x_{n}\right) V^{-1}(y) h(y) V(y) d y \\
& \quad \leqslant\left[\sup _{y \in \Delta_{n}} U_{k}^{p}\left(y, x_{n}\right) V^{-1}(y)\right] \int_{\Delta_{n}} h V=U_{k}^{p}\left(y_{n}, x_{n}\right) V^{-1}\left(y_{n}\right) \int_{\Delta_{n}} h V
\end{aligned}
$$

для некоторого $y_{n} \in \Delta_{n}$. По неравенству Гёльдера

$$
\begin{aligned}
\mathrm{I} & \leqslant \sum_{n} 2^{n} U_{k}^{q}\left(y_{n}, x_{n}\right) V^{-q / p}\left(y_{n}\right)\left(\int_{\Delta_{n}} h V\right)^{q / p} \\
& \leqslant\left(\sum_{n} 2^{n r / q} U_{k}^{r}\left(y_{n}, x_{n}\right) V^{-r / p}\left(y_{n}\right)\right)^{q / r}\left(\int_{0}^{\infty} h V\right)^{q / p}=: B_{1}^{q}\left(\int_{0}^{\infty} h V\right)^{q / p} .
\end{aligned}
$$

Имеем

$$
\begin{aligned}
B_{1}^{r} \approx & \sum_{n}\left(\int_{\Delta_{n-1}} w\right)^{r / q} U_{k}^{r}\left(y_{n}, x_{n}\right) V^{-r / p}\left(y_{n}\right) \\
\leqslant & \sum_{n}\left(\int_{\Delta_{n-1}} w(x) U_{k}^{q}\left(y_{n}, x\right) d x\right)^{r / q} V^{-r / p}\left(y_{n}\right) \\
\leqslant & \sum_{n}\left(\int_{y_{n-2}}^{y_{n}} w(x) U_{k}^{q}\left(y_{n}, x\right) d x\right)^{r / q} V^{-r / p}\left(y_{n}\right) \\
= & \sum_{k}\left(\int_{y_{2 k-2}}^{y_{2 k}} w(x) U_{k}^{q}\left(y_{2 k}, x\right) d x\right)^{r / q} V^{-r / p}\left(y_{2 k}\right) \\
& +\sum_{k}\left(\int_{y_{2 k-1}}^{y_{2 k+1}} w(x) U_{k}^{q}\left(y_{2 k+1}, x\right) d x\right)^{r / q} V^{-r / p}\left(y_{2 k+1}\right) .
\end{aligned}
$$


Если $z_{k}:=y_{2 k}$ и $z_{k}^{\prime}:=y_{2 k+1}$, то по определению $\mathbb{B}^{r}$

$$
\begin{aligned}
B_{1}^{r} \ll & \sum_{k} \\
& \left(\int_{z_{k-1}}^{z_{k}} w(x) U_{k}^{q}\left(z_{k}, x\right) d x\right)^{r / q} V^{-r / p}\left(z_{k}\right) \\
& +\sum_{k}\left(\int_{z_{k-1}^{\prime}}^{z_{k}^{\prime}} w(x) U_{k}^{q}\left(z_{k}^{\prime}, x\right) d x\right)^{r / q} V^{-r / p}\left(z_{k}^{\prime}\right) \ll \mathbb{B}^{r} .
\end{aligned}
$$

Таким образом,

$$
I \ll \mathbb{B}^{q}\left(\int_{0}^{\infty} h V\right)^{q / p} .
$$

Оценка сумм II $u$ IV. По неравенству Минковского и (3.45) находим

$$
\begin{aligned}
\mathrm{II} & \leqslant \sum_{n} 2^{n}\left(\sum_{j=n}^{\infty} k^{\alpha}\left(x_{j+1}, x_{j}\right)\left(\sum_{i=j+1}^{\infty} \int_{\Delta_{i}}\left(\int_{x_{i}}^{y} u\right)^{p} h(y) d y\right)^{\alpha / p}\right)^{q / \alpha} \\
& \approx \sum_{n} 2^{n} k^{q}\left(x_{n+1}, x_{n}\right)\left(\sum_{i=n+1}^{\infty} \int_{\Delta_{i}}\left(\int_{x_{i}}^{y} u\right)^{p} h(y) d y\right)^{q / p} \\
& \leqslant \sum_{n} 2^{n} k^{q}\left(x_{n+1}, x_{n}\right)\left(\int_{x_{n+1}}^{\infty}\left(\int_{x_{n+1}}^{y} u\right)^{p} h(y) d y\right)^{q / p}=: J_{0} .
\end{aligned}
$$

Имеем

$$
\mathrm{IV}=\sum_{n} 2^{n}\left(\sum_{i=n+2}^{\infty}\left(\sum_{j=n+1}^{i-1} k\left(x_{j}, x_{n}\right) \int_{\Delta_{j}} u\right)^{p} \int_{\Delta_{i}} h(y) d y\right)^{q / p}
$$

Применяя лемму 3.1 с $\alpha \in(0, p)$, находим

$$
k\left(x_{j}, x_{n}\right) \ll\left(\sum_{k=n}^{j-1} k^{\alpha}\left(x_{k+1}, x_{k}\right)\right)^{1 / \alpha} .
$$

По неравенству Минковского

$$
\begin{aligned}
\sum_{j=n+1}^{i-1} k\left(x_{j}, x_{n}\right) \int_{\Delta_{j}} u & \ll \sum_{j=n+1}^{i-1}\left(\sum_{k=n}^{j-1} k^{\alpha}\left(x_{k+1}, x_{k}\right)\right)^{1 / \alpha} \int_{\Delta_{j}} u \\
& \leqslant\left(\sum_{k=n}^{i-2} k^{\alpha}\left(x_{k+1}, x_{k}\right)\left(\int_{x_{k+1}}^{x_{i}} u\right)^{\alpha}\right)^{1 / \alpha}
\end{aligned}
$$

Снова по неравенству Минковского

$$
\begin{aligned}
\mathrm{IV} & \ll \sum_{n} 2^{n}\left(\sum_{i=n+2}^{\infty}\left(\sum_{k=n}^{i-2} k^{\alpha}\left(x_{k+1}, x_{k}\right)\left(\int_{x_{k+1}}^{x_{i}} u\right)^{\alpha}\right)^{p / \alpha} \int_{\Delta_{i}} h\right)^{q / p} \\
& \leqslant \sum_{n} 2^{n}\left(\sum_{k=n}^{\infty} k^{\alpha}\left(x_{k+1}, x_{k}\right)\left(\sum_{i=k+2}^{\infty}\left(\int_{x_{k+1}}^{x_{i}} u\right)^{p} \int_{\Delta_{i}} h\right)^{\alpha / p}\right)^{q / \alpha} .
\end{aligned}
$$


Далее,

$$
\begin{aligned}
\sum_{i=k+2}^{\infty}\left(\int_{x_{k+1}}^{x_{i}} u\right)^{p} \int_{\Delta_{i}} h & \leqslant \sum_{i=k+2}^{\infty} \int_{x_{i}}^{x_{i+1}} h(y)\left(\int_{x_{k+1}}^{y} u\right)^{p} d y \\
& \leqslant \int_{x_{k+1}}^{\infty}\left(\int_{x_{k+1}}^{y} u\right)^{p} h(y) d y .
\end{aligned}
$$

Следовательно, применяя (3.45), получаем

$$
\begin{aligned}
\mathrm{IV} & \ll \sum_{n} 2^{n}\left(\sum_{k=n}^{\infty} k^{\alpha}\left(x_{k+1}, x_{k}\right)\left(\int_{x_{k+1}}^{\infty}\left(\int_{x_{k+1}}^{y} u\right)^{p} h(y) d y\right)^{\alpha / p}\right)^{q / \alpha} \\
& \approx \sum_{n} 2^{n} k^{q}\left(x_{n+1}, x_{n}\right)\left(\int_{x_{n+1}}^{\infty}\left(\int_{x_{n+1}}^{y} u\right)^{p} h(y) d y\right)^{q / p}=: J_{0} .
\end{aligned}
$$

Таким образом,

$$
\mathrm{II}+\mathrm{IV} \ll J_{0} .
$$

Пусть $i \in \mathbb{Z}$. Положим

$$
A_{i}:=\left\{m \in \mathbb{Z}: 2^{i} \leqslant \sum_{n<m} 2^{n} k^{q}\left(x_{n+1}, x_{n}\right)<2^{i+1}\right\} .
$$

Обозначим $\mathscr{A}:=\bigcup_{k} A_{i_{k}} \subset \mathbb{Z}$, где $A_{i_{k}} \neq \varnothing$, и будем считать $A_{i}=\varnothing$, если $i \notin\left\{i_{k}\right\}$. Положим

$$
n_{i_{k}}:=\inf A_{i_{k}}, \quad n_{i_{k}}^{+}:=\sup A_{i_{k}} ; \quad z_{k}:=x_{n_{i_{k}}}, \quad z_{k}^{+}:=x_{n_{i_{k}}} .
$$

Тогда $n_{i_{k}}<n_{i_{k}}^{+}<n_{i_{k+1}}$ и предположим для простоты, что card $\bigcup_{k}\left\{i_{k}\right\}=\aleph_{0}$. Имеем

$$
J_{0}=\sum_{k} \sum_{n=n_{i_{k}}}^{n_{i_{k}}^{+}} 2^{n} k^{q}\left(x_{n+1}, x_{n}\right)\left(\int_{x_{n+1}}^{\infty}\left(\int_{x_{n+1}}^{y} u\right)^{p} h(y) d y\right)^{q / p}
$$

По неравенству Йенсена и (3.45)

$$
\begin{aligned}
J_{0} & \ll \sum_{k} 2^{i_{k}}\left(\int_{z_{k}}^{\infty}\left(\int_{z_{k}}^{y} u\right)^{p} h(y) d y\right)^{q / p}=\sum_{k} 2^{i_{k}}\left(\sum_{j=k}^{\infty} \int_{z_{j}}^{z_{j+1}}\left(\int_{z_{k}}^{y} u\right)^{p} h(y) d y\right)^{q / p} \\
\approx & \sum_{k} 2^{i_{k}}\left(\sum_{j=k}^{\infty} \int_{z_{j}}^{z_{j+1}}\left(\int_{z_{j}}^{y} u\right)^{p} h(y) d y\right)^{q / p} \\
& +\sum_{k} 2^{i_{k}}\left(\sum_{j=k}^{\infty}\left(\sum_{l=k}^{j-1} \int_{z_{l}}^{z_{l+1}} u\right)^{p} \int_{z_{j}}^{z_{j+1}} h\right)^{q / p} \\
\leqslant & \sum_{k} 2^{i_{k}}\left(\sum_{j=k}^{\infty} \int_{z_{j}}^{z_{j+1}}\left(\int_{z_{j}}^{y} u\right)^{p} h(y) d y\right)^{q / p} \\
+ & \sum_{k} 2^{i_{k}}\left(\sum_{j=k}^{\infty} \sum_{l=k}^{j-1}\left(\int_{z_{l}}^{z_{l+1}} u\right)^{p} \int_{z_{j}}^{z_{j+1}} h\right)^{q / p}
\end{aligned}
$$




$$
\begin{aligned}
& \approx \sum_{k} 2^{i_{k}}\left(\int_{z_{k}}^{z_{k+1}}\left(\int_{z_{k}}^{y} u\right)^{p} h(y) d y\right)^{q / p}+\sum_{k} 2^{i_{k}}\left(\sum_{l=k}^{\infty}\left(\int_{z_{l}}^{z_{l+1}} u\right)^{p} \int_{z_{l+1}}^{\infty} h\right)^{q / p} \\
& \ll \sum_{k} 2^{i_{k}}\left[\sup _{z_{k}<y<z_{k+1}}\left(\int_{z_{k}}^{y} u\right)^{p} V^{-1}(y)\right]^{q / p}\left(\int_{z_{k}}^{z_{k+1}} h V\right)^{q / p} \\
& +\sum_{k} 2^{i_{k}}\left(\int_{z_{k}}^{z_{k+1}} u\right)^{q}\left(\int_{z_{k+1}}^{\infty} h\right)^{q / p}=: J_{0,1}+J_{0,2} .
\end{aligned}
$$

По неравенству Гёльдера

$$
\begin{aligned}
J_{0,1} & \leqslant\left(\sum_{k} 2^{i_{k} r / q} \sup _{z_{k}<y<z_{k+1}}\left(\int_{z_{k}}^{y} u\right)^{r} V^{-r / p}(y)\right)^{q / r}\left(\int_{0}^{\infty} h V\right)^{q / p} \\
& =: B_{2}^{q}\left(\int_{0}^{\infty} h V\right)^{q / p} .
\end{aligned}
$$

Пусть $z_{k}^{0} \in\left[z_{k}, z_{k+1}\right]$ таковы, что

$$
\sup _{z_{k}<y<z_{k+1}}\left(\int_{z_{k}}^{y} u\right)^{r} V^{-r / p}(y)=\left(\int_{z_{k}}^{z_{k}^{0}} u\right)^{r} V^{-r / p}\left(z_{k}^{0}\right) .
$$

Тогда

$$
B_{2}^{r}=\sum_{k} 2^{i_{k} r / q}\left(\int_{z_{k}}^{z_{k}^{0}} u\right)^{r} V^{-r / p}\left(z_{k}^{0}\right) .
$$

Из (3.53) следует, что

$$
\begin{aligned}
2^{i_{k}} & \leqslant \sum_{n<n_{i_{k}}} 2^{n} k^{q}\left(x_{n+1}, x_{n}\right)=\sum_{n_{i_{k-2}} \leqslant n<n_{i_{k}}} 2^{n} k^{q}\left(x_{n+1}, x_{n}\right)+\sum_{n<n_{i_{k}-2}} 2^{n} k^{q}\left(x_{n+1}, x_{n}\right) \\
& \leqslant \sum_{n_{i_{k-2}} \leqslant n<n_{i_{k}}} 2^{n} k^{q}\left(x_{n+1}, x_{n}\right)+2^{i_{k-2}+1} .
\end{aligned}
$$

Таким образом,

$$
2^{i_{k}} \leqslant 2 \sum_{n_{i_{k-2}} \leqslant n<n_{i_{k}}} 2^{n} k^{q}\left(x_{n+1}, x_{n}\right)
$$

и

$$
B_{2}^{r} \ll \sum_{k}\left(\sum_{n_{i_{k}-2} \leqslant n<n_{i_{k}}} 2^{n} k^{q}\left(x_{n+1}, x_{n}\right)\right)^{r / q}\left(\int_{z_{k}}^{z_{k}^{0}} u\right)^{r} V^{-r / p}\left(z_{k}^{0}\right) .
$$

Имеем

$$
\begin{aligned}
\sum_{n_{i_{k-2}} \leqslant n<n_{i_{k}}} & 2^{n} k^{q}\left(x_{n+1}, x_{n}\right) \approx \sum_{n_{i_{k-2}} \leqslant n<n_{i_{k}}} k^{q}\left(x_{n+1}, x_{n}\right) \int_{x_{n-1}}^{x_{n}} w(y) d y \\
& \ll \sum_{n_{i_{k-2}} \leqslant n<n_{i_{k}}} \int_{x_{n-1}}^{x_{n}} k^{q}\left(x_{n+1}, y\right) w(y) d y \ll \int_{z_{k-2}-1}^{z_{k}} k^{q}\left(z_{k}, y\right) w(y) d y \\
& \leqslant \int_{z_{k-3}}^{z_{k}} k^{q}\left(z_{k}, y\right) w(y) d y=: \varphi_{k} .
\end{aligned}
$$


Далее,

$$
\begin{aligned}
\varphi_{k}^{1 / q}\left(\int_{z_{k}}^{z_{k}^{0}} u\right) & =\left(\int_{z_{k-3}}^{z_{k}} w(y)\left(k\left(z_{k}, y\right) \int_{z_{k}}^{z_{k}^{0}} u(z) d z\right)^{q} d y\right)^{1 / q} \\
& \ll\left(\int_{z_{k-3}}^{z_{k}} w(y)\left(\int_{z_{k}}^{z_{k}^{0}} k(z, y) u(z) d z\right)^{q} d y\right)^{1 / q} \\
& \leqslant\left(\int_{z_{k-3}}^{z_{k}^{0}} w(y)\left(\int_{y}^{z_{k}^{0}} k(z, y) u(z) d z\right)^{q} d y\right)^{1 / q} .
\end{aligned}
$$

Поскольку $z_{k-3} \geqslant z_{k-4}^{0}$, то

$$
B_{2}^{r} \ll \sum_{k}\left(\int_{z_{k-4}^{0}}^{z_{k}^{0}} w(y)\left(\int_{y}^{z_{k}^{0}} k(z, y) u(z) d z\right)^{q} d y\right)^{r / q} V^{-r / p}\left(z_{k}^{0}\right) .
$$

Используя обычную процедуру, а именно разбивая сумму $\sum_{k}$ в сумму сумм $\sum_{k=2 n}+\sum_{k=2 n+1}$ дважды, мы видим, что $B_{2} \ll \mathbb{B}$.

Теперь докажем оценку сверху для $J_{0,2}$. Имеем

$$
J_{0,2}=\sum_{k} 2^{i_{k}}\left(\int_{z_{k}}^{z_{k+1}} u\right)^{q}\left(\int_{z_{k+1}}^{\infty} h\right)^{q / p}
$$

Пусть $j \in \mathbb{Z}$ и

$$
B_{j}:=\left\{m \in \mathbb{Z}: 2^{j} \leqslant \sum_{k<m} 2^{i_{k}}\left(\int_{z_{k}}^{z_{k+1}} u\right)^{q}<2^{j+1}\right\} .
$$

Обозначим $\mathscr{B}:=\bigcup_{k} B_{j_{k}} \subset \mathbb{Z}$, где $B_{j_{k}} \neq \varnothing$, и $B_{j}=\varnothing$, если $j \notin\left\{j_{k}\right\}$. Положим

$$
l_{j_{k}}:=\inf B_{j_{k}}, \quad l_{j_{k}}^{+}:=\sup B_{j_{k}} ; \quad y_{k}:=z_{l_{j_{k}}}, \quad y_{k}^{+}:=z_{l_{j_{k}}^{+}} .
$$

Тогда $l_{j_{k}}<l_{j_{k}}^{+}<l_{j_{k+1}}$ и будем для простоты считать, что card $\bigcup_{k}\left\{j_{k}\right\}=\aleph_{0}$. По неравенству Гёльдера

$$
\begin{aligned}
J_{0,2} & =\sum_{j} \sum_{k=l_{j}}^{l_{j}^{+}} 2^{i_{k}}\left(\int_{z_{k}}^{z_{k+1}} u\right)^{q}\left(\int_{z_{k+1}}^{\infty} h\right)^{q / p} \ll \sum_{j} 2^{j}\left(\int_{y_{j}}^{\infty} h\right)^{q / p} \\
& =\sum_{j} 2^{j}\left(\sum_{k=j}^{\infty} \int_{y_{k}}^{y_{k}^{+}} h\right)^{q / p} \approx \sum_{k} 2^{k}\left(\int_{y_{k}}^{y_{k}^{+}} h\right)^{q / p} \\
& \leqslant \sum_{k} 2^{k} V^{-q / p}\left(y_{k}\right)\left(\int_{y_{k}}^{y_{k}^{+}} h V\right)^{q / p} \leqslant\left(\sum_{k} 2^{k r / q} V^{-r / p}\left(y_{k}\right)\right)^{q / r}\left(\int_{0}^{\infty} h V\right)^{q / p} \\
& =: B_{3}^{q}\left(\int_{0}^{\infty} h V\right)^{q / p} .
\end{aligned}
$$


Из (3.56) следует, что $2^{j} \leqslant 2 \sum_{l_{j-2} \leqslant k<l_{j}} 2^{i_{k}}\left(\int_{z_{k}}^{z_{k+1}} u\right)^{q}$. Следовательно,

$$
B_{3}^{r} \ll \sum_{j}\left(\sum_{l_{j-2} \leqslant k<l_{j}} 2^{i_{k}}\left(\int_{z_{k}}^{z_{k+1}} u\right)^{q}\right)^{r / q} V^{-r / p}\left(y_{j}\right) .
$$

Используя (3.54) и (3.55), находим

$$
\begin{aligned}
B_{3}^{r} & \ll \sum_{j}\left(\sum_{l_{j-2} \leqslant k<l_{j}} \int_{z_{k-3}}^{z_{k}}\left(\int_{y}^{z_{k+1}} k(z, y) u(z) d z\right)^{q} w(y) d y\right)^{r / q} V^{-r / p}\left(y_{j}\right) \\
& \leqslant \sum_{j}\left(\sum_{l_{j-2} \leqslant k<l_{j}} \int_{z_{k-3}}^{z_{k}}\left(\int_{y}^{y_{j}} k(z, y) u(z) d z\right)^{q} w(y) d y\right)^{r / q} V^{-r / p}\left(y_{j}\right) \\
& \leqslant \sum_{j}\left(\int_{y_{j-8}}^{y_{j}}\left(\int_{y}^{y_{j}} k(z, y) u(z) d z\right)^{q} w(y) d y\right)^{r / q} V^{-r / p}\left(y_{j}\right)
\end{aligned}
$$

отсюда следует оценка $B_{3} \ll \mathbb{B}$.

Оценка суммы III. Так как $p \in(0,1]$, то по неравенству Йенсена и $(3.45)$ имеем

$$
\begin{aligned}
\mathrm{III} & \leqslant \sum_{n} 2^{n}\left(\sum_{i=n+1}^{\infty} \sum_{j=n}^{i-1} U_{k}^{p}\left(x_{j+1}, x_{j}\right) \int_{\Delta_{i}} h\right)^{q / p} \\
& =\sum_{n} 2^{n}\left(\sum_{j=n}^{\infty} U_{k}^{p}\left(x_{j+1}, x_{j}\right) \int_{x_{j+1}}^{\infty} h\right)^{q / p} \\
& \approx \sum_{n} 2^{n} U_{k}^{q}\left(x_{n+1}, x_{n}\right)\left(\int_{x_{n+1}}^{\infty} h\right)^{q / p}=: J_{1} .
\end{aligned}
$$

Пусть $k \in \mathbb{Z}$ и

$$
G_{k}:=\left\{m \in \mathbb{Z}: 2^{k} \leqslant \sum_{n<m} 2^{n}\left(\int_{\Delta_{n}} k\left(z, x_{n}\right) u(z) d z\right)^{q}<2^{k+1}\right\} .
$$

Как и ранее, считаем для простоты, что $G_{k}$ непусты, и полагаем

$$
\lambda_{k}:=\inf G_{k}, \quad \lambda_{k}^{+}:=\sup G_{k}<\lambda_{k+1} .
$$

Тогда

$$
\begin{aligned}
J_{1} & =\sum_{k} \sum_{\lambda_{k} \leqslant n<\lambda_{k}^{+}} 2^{n}\left(\int_{\Delta_{n}} k\left(z, x_{n}\right) u(z) d z\right)^{q}\left(\int_{x_{n+1}}^{\infty} h\right)^{q / p} \ll \sum_{k} 2^{k}\left(\int_{x_{\lambda_{k}+1}}^{\infty} h\right)^{q / p} \\
& \leqslant \sum_{k} 2^{k}\left(\sum_{j=k}^{\infty} \int_{x_{\lambda_{j}}}^{x_{\lambda_{j+1}}} h\right)^{q / p} \approx \sum_{k} 2^{k}\left(\int_{x_{\lambda_{k}}}^{x_{\lambda_{k+1}}} h\right)^{q / p}
\end{aligned}
$$




$$
\begin{aligned}
& \leqslant \sum_{k} 2^{k} V^{-q / p}\left(x_{\lambda_{k}}\right)\left(\int_{x_{\lambda_{k}}}^{x_{\lambda_{k+1}}} h V\right)^{q / p} \leqslant\left(\sum_{k} 2^{k r / q} V^{-r / p}\left(x_{\lambda_{k}}\right)\right)^{q / r}\left(\int_{0}^{\infty} h V\right)^{q / p} \\
& =: B_{4}^{q}\left(\int_{0}^{\infty} h V\right)^{q / p} .
\end{aligned}
$$

Применяя

$$
\begin{aligned}
2^{k} & \ll \sum_{\lambda_{k-2} \leqslant n<\lambda_{k}} 2^{n}\left(\int_{\Delta_{n}} k\left(z, x_{n}\right) u(z) d z\right)^{q} \\
& \approx \sum_{\lambda_{k-2} \leqslant n<\lambda_{k}} \int_{\Delta_{n-1}} w(y) d y\left(\int_{\Delta_{n}} k\left(z, x_{n}\right) u(z) d z\right)^{q} \\
& \ll \sum_{\lambda_{k-2} \leqslant n<\lambda_{k}} \int_{\Delta_{n-1}}\left(\int_{y}^{x_{\lambda_{k}}} k(z, y) u(z) d z\right)^{q} w(y) d y \\
& \leqslant \int_{x_{\lambda_{k-3}}}^{x_{\lambda_{k}}}\left(\int_{y}^{x_{\lambda_{k}}} k(z, y) u(z) d z\right)^{q} w(y) d y,
\end{aligned}
$$

мы видим, что

$$
B_{4}^{r} \ll \sum_{k}\left(\int_{x_{\lambda_{k-3}}}^{x_{\lambda_{k}}}\left(\int_{y}^{x_{\lambda_{k}}} k(z, y) u(z) d z\right)^{q} w(y) d y\right) V_{x_{\lambda_{k}}}^{-r / p} \ll \mathbb{B}^{r} .
$$

Таким образом, доказательство импликации $(3.50) \Rightarrow(3.47)$ завершено.

$(3.47) \Rightarrow(3.46)$ следует по неравенству Минковского.

$(3.46) \Rightarrow(3.48) \Rightarrow(3.49)$ доказываются элементарно.

Докажем последний шаг (3.49) $\Rightarrow(3.50)$. Для этого предположим, что $\left\{x_{k}\right\} \subset$ $\mathbb{R}_{+}-$произвольная возрастающая последовательность. Пусть $\varepsilon_{k} \in\left(x_{k}, x_{k+1}\right)$ таковы, что $V\left(\varepsilon_{k}\right) \leqslant 2 V\left(x_{k}\right)$. Положим

$$
h(x):=\sum_{k} \frac{a_{k}}{\varepsilon_{k}-x_{k}} \frac{\chi_{\left(x_{k}, \varepsilon_{k}\right)}(x)}{V\left(x_{k}\right)},
$$

где $\left\{a_{k}\right\} \subset[0, \infty)$ таковы, что $\sum_{k} a_{k}<\infty$. Тогда

$$
\int_{0}^{\infty} h V=\sum_{k} \frac{a_{k}}{\left(\varepsilon_{k}-x_{k}\right) V\left(x_{k}\right)} \int_{x_{k}}^{\varepsilon_{k}} V(x) d x \leqslant \sum_{k} \frac{a_{k} V\left(\varepsilon_{k}\right)}{V\left(x_{k}\right)} \leqslant 2 \sum_{k} a_{k} .
$$

Запишем

$$
\begin{aligned}
H & :=\int_{0}^{\infty}\left[\sup _{y \geqslant x}\left(\int_{x}^{y} k(z, x) u(z) d z\right)^{p} \int_{y}^{\infty} h\right]^{q / p} w(x) d x \\
& =\sum_{k} \int_{x_{k}}^{x_{k+1}}\left[\sup _{y \geqslant x}\left(\int_{x}^{y} k(z, x) u(z) d z\right)^{p} \int_{y}^{\infty} h\right]^{q / p} w(x) d x \\
& \geqslant \sum_{k} \int_{x_{k}}^{x_{k+1}}\left(\int_{x}^{x_{k+1}} k(z, x) u(z) d z\right)^{q} w(x) d x \int_{x_{k+1}}^{\infty} h .
\end{aligned}
$$


Имеем

$$
\int_{x_{k+1}}^{\infty} h=\sum_{n \geqslant k+1} \frac{a_{n}}{V\left(x_{n}\right)} \geqslant \frac{a_{k+1}}{V\left(x_{k+1}\right)} .
$$

Следовательно,

$$
\begin{aligned}
H & \geqslant \sum_{k}\left(\int_{x_{k}}^{x_{k+1}}\left(\int_{x}^{x_{k+1}} k(z, x) u(z) d z\right)^{q} w(x) d x\right) \frac{a_{k+1}^{q / p}}{V^{q / p}\left(x_{k+1}\right)} \\
& =: \sum_{k} b_{k} a_{k+1}^{q / p} .
\end{aligned}
$$

Тогда (3.49) влечет

$$
\left(\sum_{k} b_{k} a_{k+1}^{q / p}\right)^{p / q} \ll C_{4}^{p} \sum_{k} a_{k+1}
$$

для всех $\left\{a_{k}\right\} \subset \mathbb{R}_{+}$. Отсюда

$$
\left(\sum_{k} b_{k}^{r / q}\right)^{p / r} \ll C_{4}^{p},
$$

что эквивалентно $\mathbb{B} \ll C_{4}$. Теорема 3.1 доказана.

Следующая теорема посвящена важному частному случаю теоремы 3.11 , когда $k(x, y) \equiv 1$ и критерию удается придать интегральный вид. Этот случай изучался М. Л. Гольдманом [33], [34]. Мы получаем критерий в другой форме и приводим более простое доказательство. Обозначим $\varphi(x):=W^{-1}(4 W(x))$, где $W^{-1}(t):=\inf \{s \geqslant 0: W(s)=t\}-$ обратная функция к $W(t):=\int_{0}^{t} w$.

Теорема 3.12. Пусть $0<q<p \leqslant 1$. Следующие условия эквивалентны:

$$
\begin{aligned}
& \left(\int_{0}^{\infty}\left(\int_{x}^{\infty} f u\right)^{q} w(x) d x\right)^{1 / q} \leqslant C_{1}\left(\int_{0}^{\infty} f^{p} v\right)^{1 / p}, \quad f \in \mathfrak{M}^{\downarrow}, \\
& \left(\int_{0}^{\infty}\left(\int_{x}^{\infty} U^{p}(y, x) h(y) d y\right)^{q / p} w(x) d x\right)^{p / q} \leqslant C_{2}^{p} \int_{0}^{\infty} h V, \quad h \in \mathfrak{M}^{+}, \quad \\
& \left(\int_{0}^{\infty}\left(\sup _{y \geqslant x} U^{p}(y, x) \int_{y}^{\infty} h\right)^{q / p} w(x) d x\right)^{p / q} \leqslant C_{3}^{p} \int_{0}^{\infty} h V, \quad h \in \mathfrak{M}^{+}, \\
& \left(\int_{0}^{\infty}\left(\sup _{y \geqslant x} U(y, x) f(y)\right)^{q} w(x) d x\right)^{1 / q} \leqslant C_{4}\left(\int_{0}^{\infty} f^{p} v\right)^{1 / p}, \quad f \in \mathfrak{M}^{\downarrow}, \\
& \left(\int_{0}^{\infty}\left(\sup _{x \leqslant y \leqslant \varphi(x)} U^{p}(y, x) \int_{y}^{\infty} h\right)^{q / p} w(x) d x\right)^{p / q} \leqslant C_{5}^{p} \int_{0}^{\infty} h V, \quad h \in \mathfrak{M}^{+}, \\
& \left(\int_{0}^{\infty}\left(\sup _{x \leqslant y \leqslant \varphi(x)} U(y, x) f(y)\right)^{q} w(x) d x\right)^{1 / q} \leqslant C_{6}\left(\int_{0}^{\infty} f^{p} v\right)^{1 / p}, \quad f \in \mathfrak{M}^{\downarrow},
\end{aligned}
$$




$$
\begin{gathered}
\left(\int_{0}^{\infty}\left[\left(\int_{x}^{\varphi(x)} U^{p}(y, x) h(y) d y\right)^{q / p}+U^{q}(\varphi(x), x)\left(\int_{\varphi(x)}^{\infty} h\right)^{q / p}\right] w(x) d x\right)^{p / q} \\
\leqslant C_{7}^{p} \int_{0}^{\infty} h V
\end{gathered}
$$

для всех $h \in \mathfrak{M}^{+}$,

$$
\begin{gathered}
\mathbb{B}^{r}:=\sup _{\left\{x_{k}\right\}} \sum_{k \in \mathbb{Z}}\left(\int_{x_{k}}^{x_{k+1}} U^{q}\left(x_{k+1}, y\right) w(y) d y\right)^{r / q} V^{-r / p}\left(x_{k+1}\right)<\infty, \\
\mathbf{B}_{1}+\mathbf{B}_{2}<\infty,
\end{gathered}
$$

¿əe

$$
\begin{gathered}
\mathbf{B}_{1}^{r}:=\int_{0}^{\infty} \sup _{x \leqslant y \leqslant \varphi(x)} U^{r}(y, x) V^{-r / p}(y) W^{r / p}(x) w(x) d x \\
\mathbf{B}_{2}^{r}:=\int_{0}^{\infty}\left(\int_{0}^{x} w(y) U^{q}(\varphi(y), y) d y\right)^{r / p} V^{-r / p}(\varphi(x)) w(x) U^{q}(\varphi(x), x) d x .
\end{gathered}
$$

Более того,

$$
C_{1} \approx C_{2} \approx C_{3}=C_{4} \approx C_{5} \approx C_{6} \approx C_{7} \approx \mathbb{B} \approx \mathbf{B}_{1}+\mathbf{B}_{2}
$$

ДокАЗАтЕльство. Заметим сначала, что эквивалентность $(3.60) \Leftrightarrow(3.64)$ следует из теоремы 4.4 работы [110]. Далее, импликация (3.60) $\Rightarrow(3.59)$ очевидна, а для доказательства $(3.59) \Rightarrow(3.60)$ надо применить предложение 2.1 и теорему Фату. Для любой функции $f \in \mathfrak{M}^{\downarrow}$ имеем

$$
\int_{x}^{\infty} f u \geqslant \sup _{y \geqslant x} \int_{x}^{y} f u \geqslant \sup _{y \geqslant x} U(y, x) f(y) .
$$

Отсюда следует, что $(3.57) \Rightarrow(3.60)$. Неравенство (3.57) эквивалентно следующему:

$$
\left(\int_{0}^{\infty}\left(\int_{x}^{\infty} f^{1 / p} u\right)^{q} w\right)^{p / q} \leqslant C_{1}^{p}\left(\int_{0}^{\infty} f v\right)^{1 / p}, \quad f \in \mathfrak{M}^{\downarrow} .
$$

Если $f(x)=\int_{x}^{\infty} h$, то по неравенству Минковского

$$
\int_{x}^{\infty} f^{1 / p} u=\int_{x}^{\infty}\left(\int_{z}^{\infty} h\right)^{1 / p} u(z) d z \leqslant\left(\int_{x}^{\infty} U^{p}(y, x) h(y) d y\right)^{1 / p}
$$

и $(3.58) \Rightarrow(3.57)$ доказывается применением предложения 2.1.

Покажем, что $(3.59) \Rightarrow(3.58)$. Для этого обозначим левые части (3.58) и (3.59) через $A^{p / q}$ и $B^{p / q}$ соответственно. Предположим, что (3.59) выполняется. Тогда

$$
A:=\sum_{n} \int_{\Delta_{n}}\left(\int_{x}^{\infty} U^{p}(y, x) h(y) d y\right)^{q / p} w(x) d x
$$




$$
\begin{aligned}
& \approx \sum_{n} 2^{n}\left(\int_{\Delta_{n}} U^{p}\left(y, x_{n}\right) h(y) d y\right)^{q / p} \\
& \quad+\sum_{n} 2^{n}\left(\sum_{i=n+1}^{\infty} U^{p}\left(x_{i}, x_{n}\right) \int_{\Delta_{i}} h(y) d y\right)^{q / p}=: A_{1}+A_{2} .
\end{aligned}
$$

Используя неравенство Йенсена и (3.45), получаем

$$
\begin{aligned}
A_{2} & :=\sum_{n} 2^{n}\left(\sum_{i=n+1}^{\infty}\left(\sum_{j=n}^{i-1} U\left(x_{j+1}, x_{j}\right)\right)^{p} \int_{\Delta_{i}} h(y) d y\right)^{q / p} \\
& \ll \sum_{n} 2^{n}\left(U^{p}\left(x_{n+1}, x_{n}\right) \int_{x_{n+1}}^{\infty} h(y) d y\right)^{q / p} .
\end{aligned}
$$

Аналогично, для константы $B$ имеем

$$
\begin{aligned}
B:= & \int_{0}^{\infty}\left(\sup _{y \geqslant x} U^{p}(y, x) \int_{y}^{\infty} h\right)^{q / p} w(x) d x \\
= & \sum_{n} \int_{\Delta_{n}}\left(\sup _{y \geqslant x} U^{p}(y, x) \int_{y}^{\infty} h\right)^{q / p} w(x) d x \approx \sum_{n} 2^{n}\left(\sup _{y \geqslant x_{n}} U^{p}\left(y, x_{n}\right) \int_{y}^{\infty} h\right)^{q / p} \\
= & \sum_{n} 2^{n}\left(\sup _{i \geqslant n} \sup _{y \in \Delta_{i}} U^{p}\left(y, x_{n}\right) \int_{y}^{\infty} h\right)^{q / p} \approx \sum_{n} 2^{n}\left(\sup _{i \geqslant n} \sup _{y \in \Delta_{i}} U^{p}\left(y, x_{i}\right) \int_{y}^{\infty} h\right)^{q / p} \\
& +\sum_{n} 2^{n}\left(\sup _{i \geqslant n+1} U^{p}\left(x_{i}, x_{n}\right) \int_{x_{i}}^{\infty} h\right)^{q / p} \approx \sum_{n} 2^{n}\left(\sup _{y \in \Delta_{n}} U^{p}\left(y, x_{n}\right) \int_{y}^{\infty} h\right)^{q / p} \\
& +\sum_{n} 2^{n}\left(\sup _{i \geqslant n+1} U^{p}\left(x_{i}, x_{n}\right) \int_{x_{i}}^{\infty} h\right)^{q / p}=: B_{1}+B_{2} .
\end{aligned}
$$

Если (3.59) выполняется, то

$$
B_{i} \ll C_{3}^{q}\left(\int_{0}^{\infty} h V\right)^{q / p}, \quad i=1,2 .
$$

Из (3.71) следует, что

$$
A_{2} \ll B_{2} \ll C_{3}^{q}\left(\int_{0}^{\infty} h V\right)^{q / p} .
$$

По неравенству Гёльдера

$$
\begin{aligned}
A_{1} & \approx \sum_{n} 2^{n}\left(\int_{\Delta_{n}} U^{p}\left(y, x_{n}\right) V^{-1}(y) h(y) V(y) d y\right)^{q / p} \\
& \leqslant \sum_{n} 2^{n}\left(\sup _{y \in \Delta_{n}} U^{p}\left(y, x_{n}\right) V^{-1}(y)\right)^{q / p}\left(\int_{\Delta_{n}} h V\right)^{q / p}
\end{aligned}
$$




$$
\begin{aligned}
& \leqslant\left(\sum_{n} 2^{n r / q}\left(\sup _{y \in \Delta_{n}} U^{p}\left(y, x_{n}\right) V^{-1}(y)\right)^{r / p}\right)^{q / r}\left(\sum_{n} \int_{\Delta_{n}} h V\right)^{q / p} \\
& =: \mathbb{D}^{q}\left(\int_{0}^{\infty} h V\right)^{q / p} .
\end{aligned}
$$

Из (3.72) получаем

$$
\sum_{n} 2^{n}\left(\sup _{y \in \Delta_{n}} U^{p}\left(y, x_{n}\right) \int_{y}^{x_{n+1}} h\right)^{q / p} \ll C_{3}^{q}\left(\int_{0}^{\infty} h V\right)^{q / p} .
$$

Пусть $H_{n}: L_{V}^{1}\left[\Delta_{n}\right] \rightarrow L^{\infty}\left[\Delta_{n}\right]-$ оператор вида

$$
H_{n} h(y):=U^{p}\left(y, x_{n}\right) \int_{y}^{x_{n+1}} h .
$$

Тогда по теореме 1.1

$$
d_{n}:=\left\|H_{n}\right\|_{L_{V}^{1}\left[\Delta_{n}\right] \rightarrow L^{\infty}\left[\Delta_{n}\right]}=\sup _{y \in \Delta_{n}} U^{p}\left(y, x_{n}\right) V^{-1}(y) .
$$

Пусть $h_{n} \in L_{V}^{1}\left[\Delta_{n}\right]$ - такая функция, что

$$
\sup _{y \in \Delta_{n}} U^{p}\left(y, x_{n}\right) \int_{y}^{x_{n+1}} h_{n} \geqslant \frac{d_{n}}{2} \int_{\Delta_{n}} h_{n} V .
$$

Тогда из (3.74) следует

$$
\begin{gathered}
C_{3}^{q} \gg \sup _{h \geqslant 0} \frac{\sum_{n} 2^{n}\left(\sup _{y \in \Delta_{n}} U^{p}\left(y, x_{n}\right) \int_{y}^{x_{n+1}} h\right)^{q / p}}{\left(\int_{0}^{\infty} h V\right)^{q / p}} \\
\geqslant \sup _{h=\sum_{n} a_{n} h_{n}} \frac{\sum_{n} 2^{n} a_{n}^{q / p}\left(\sup _{y \in \Delta_{n}} U^{p}\left(y, x_{n}\right) \int_{y}^{x_{n+1}} h\right)^{q / p}}{\left(\sum_{n} a_{n} \int_{\Delta_{n}} h V\right)^{q / p}} \\
\gg \sup _{h=\sum_{n} a_{n} h_{n}} \frac{\sum_{n} 2^{n} d_{n}^{q / p}\left(a_{n} \int_{\Delta_{n}} h V\right)^{q / p}}{\left(\sum_{n} a_{n} \int_{\Delta_{n}} h V\right)^{q / p}}=\mathbb{D}^{q} .
\end{gathered}
$$

Следовательно, $\mathbb{D} \ll C_{3}$ и

$$
A_{1} \ll \mathbb{D}^{q}\left(\int_{0}^{\infty} h V\right)^{q / p} \ll C_{3}^{q}\left(\int_{0}^{\infty} h V\right)^{q / p} .
$$

Отсюда и из (3.73) вытекает, что $(3.59) \Rightarrow(3.58)$.

Для доказательства остальных эквивалентностей заметим, что

$$
C_{5} \leqslant C_{3}, \quad C_{7} \leqslant C_{2}, \quad C_{6} \leqslant C_{4}, \quad C_{6}=C_{5} \leqslant C_{7} .
$$


Поэтому, если показать, что $C_{2} \ll C_{5}$, то ясно, что $C_{2} \approx C_{5} \approx C_{6} \approx C_{7}$. Как показано выше,

$$
A:=\int_{0}^{\infty}\left(\int_{x}^{\infty} U^{p}(y, x) h(y) d y\right)^{q / p} w(x) d x \approx A_{1}+A_{2},
$$

причем

$$
A_{2} \ll \sum_{n} 2^{n} U^{q}\left(x_{n+1}, x_{n}\right)\left(\int_{x_{n+1}}^{\infty} h\right)^{q / p} .
$$

Так как $\varphi\left(x_{n-1}\right)=x_{n+1}$, то

$$
\begin{aligned}
& U^{q}\left(x_{n+1}, x_{n}\right)\left(\int_{x_{n+1}}^{\infty} h\right)^{q / p}=\sup _{x_{n} \leqslant y \leqslant x_{n+1}} U^{q}\left(y, x_{n}\right)\left(\int_{x_{n+1}}^{\infty} h\right)^{q / p} \\
& \leqslant \sup _{x_{n} \leqslant y \leqslant x_{n+1}} U^{q}\left(y, x_{n}\right)\left(\int_{y}^{\infty} h\right)^{q / p}=\sup _{x_{n} \leqslant y \leqslant \varphi\left(x_{n-1}\right)} U^{q}\left(y, x_{n}\right)\left(\int_{y}^{\infty} h\right)^{q / p} \\
& \leqslant \sup _{x \leqslant y \leqslant \varphi(x)} U^{q}(y, x)\left(\int_{y}^{\infty} h\right)^{q / p}, \quad x \in\left(x_{n-1}, x_{n}\right) .
\end{aligned}
$$

Тогда

$$
\begin{aligned}
A_{2} & \ll \sum_{n}\left(\int_{x_{n-1}}^{x_{n}} w\right) U^{q}\left(x_{n+1}, x_{n}\right)\left(\int_{x_{n+1}}^{\infty} h\right)^{q / p} \\
& \leqslant \sum_{n} \int_{x_{n-1}}^{x_{n}}\left[\sup _{x \leqslant y \leqslant \varphi(x)} U^{p}(y, x)\left(\int_{y}^{\infty} h\right)\right]^{q / p} w(x) d x \\
& \leqslant \int_{0}^{\infty}\left[\sup _{x \leqslant y \leqslant \varphi(x)} U^{p}(y, x)\left(\int_{y}^{\infty} h\right)\right]^{q / p} w(x) d x \leqslant C_{5}^{q}\left(\int_{0}^{\infty} h V\right)^{q / p} .
\end{aligned}
$$

Из $x_{n-1} \leqslant x \leqslant x_{n}$ следует, что $x_{n+1}=\varphi\left(x_{n-1}\right) \leqslant \varphi(x)$, поэтому

$$
\begin{aligned}
& \sup _{x \leqslant y \leqslant \varphi(x)} U^{p}(y, x)\left(\int_{y}^{\infty} h\right) \geqslant \sup _{x_{n} \leqslant y \leqslant \varphi\left(x_{n-1}\right)} U^{p}(y, x)\left(\int_{y}^{\infty} h\right) \\
& \sup _{x_{n} \leqslant y \leqslant x_{n+1}} U^{p}(y, x)\left(\int_{y}^{\infty} h\right) \geqslant \sup _{x_{n} \leqslant y \leqslant x_{n+1}} U^{p}\left(y, x_{n}\right)\left(\int_{y}^{x_{n+1}} h\right) .
\end{aligned}
$$

Отсюда и из предыдущей оценки вытекает

$$
\sum_{n} 2^{n} \sup _{x_{n} \leqslant y \leqslant x_{n+1}} U^{q}\left(y, x_{n}\right)\left(\int_{y}^{x_{n+1}} h\right)^{q / p} \ll C_{5}^{q}\left(\int_{y}^{\infty} h V\right)^{q / p} .
$$

Рассуждая так же, как после неравенства (3.74), получим

$$
A_{1} \ll C_{5}^{q}\left(\int_{y}^{\infty} h V\right)^{q / p}
$$

Следовательно, $C_{2} \ll C_{5}$. 
Далее, мы покажем, что

$$
C_{2} \ll \mathbf{B}_{1}+\mathbf{B}_{2} .
$$

Из (3.75) мы знаем, что

$$
A_{1} \ll \mathbb{D}^{q}\left(\int_{y}^{\infty} h V\right)^{q / p}
$$

где

$$
\mathbb{D}^{r}:=\sum_{n} 2^{n r / q}\left(\sup _{y \in \Delta_{n}} U^{p}\left(y, x_{n}\right) V^{-1}(y)\right)^{r / p} .
$$

Так как

$$
\begin{aligned}
\mathbb{D}^{r} & \approx \sum_{n} \int_{\Delta_{n-1}} W^{r / p} w\left(\sup _{y \in \Delta_{n}} U^{p}\left(y, x_{n}\right) V^{-1}(y)\right)^{r / p} \\
& \leqslant \sum_{n} \int_{\Delta_{n-1}} W^{r / p}(x) w(x)\left(\sup _{x \leqslant y \leqslant x_{n+1}} U^{p}(y, x) V^{-1}(y)\right)^{r / p} d x \\
& \leqslant \sum_{n} \int_{\Delta_{n-1}} W^{r / p}(x) w(x)\left(\sup _{x \leqslant y \leqslant \varphi(x)} U^{p}(y, x) V^{-1}(y)\right)^{r / p} d x \leqslant \mathbf{B}_{1}^{r},
\end{aligned}
$$

TO

$$
A_{1} \ll \mathbf{B}_{1}^{q}\left(\int_{y}^{\infty} h V\right)^{q / p}
$$

Далее,

$$
\begin{aligned}
A_{2} \ll & \sum_{n}\left(\int_{\Delta_{n-1}} w\right) U^{q}\left(x_{n+1}, x_{n}\right)\left(\int_{x_{n+2}}^{\infty} h\right)^{q / p} \\
& +\sum_{n} 2^{n} U^{q}\left(x_{n+1}, x_{n}\right)\left(\int_{\Delta_{n+1}}^{\infty} h\right)^{q / p} \\
\leqslant & \sum_{n} \int_{\Delta_{n-1}} w(x) U^{q}(\varphi(x), x)\left(\int_{\varphi(x)}^{\infty} h\right)^{q / p} d x \\
& +\sum_{n} 2^{n} U^{q}\left(x_{n+1}, x_{n}\right) V^{-q / p}\left(x_{n+1}\right)\left(\int_{\Delta_{n+1}}^{\infty} h V\right)^{q / p} \\
\leqslant & \int_{0}^{\infty} U^{q}(\varphi(x), x)\left(\int_{\varphi(x)}^{\infty} h\right)^{q / p} w(x) d x \\
& +\left(\sum_{n} 2^{n r / q} U^{r}\left(x_{n+1}, x_{n}\right) V^{-r / p}\left(x_{n+1}\right)\right)^{q / r}\left(\int_{0}^{\infty} h V\right)^{q / p} .
\end{aligned}
$$

Применяя замечание 1.2 и (3.78), находим

$$
A_{2} \ll \mathbf{B}_{2}^{q}\left(\int_{0}^{\infty} h V\right)^{q / p}+\mathbf{B}_{1}^{q}\left(\int_{0}^{\infty} h V\right)^{q / p}
$$

и (3.77) доказано. 
Из (3.63) вытекает, что

$$
\left(\int_{0}^{\infty} U^{q}(\varphi(x), x)\left(\int_{\varphi(x)}^{\infty} h\right)^{q / p} w(x) d x\right)^{p / q} \leqslant C_{6}^{p} \int_{0}^{\infty} h V .
$$

Отсюда и из замечания 1.2 следует, что $\mathbf{B}_{2} \ll C_{6}$. Так как $C_{5} \approx C_{6}$, то требуемый результат будет доказан, если мы покажем, что $\mathbf{B}_{1} \ll C_{5}$. Имеем

$$
\begin{aligned}
\mathbf{B}_{1}^{r} & \ll \sum_{n} 2^{n r / q} \sup _{x_{n} \leqslant y \leqslant \varphi\left(x_{n+1}\right)} U^{r}\left(y, x_{n}\right) V^{-r / p}(y) \\
& =\sum_{n} 2^{n r / q} \sup _{x_{n} \leqslant y \leqslant x_{n+3}} U^{r}\left(y, x_{n}\right) V^{-r / p}(y) \\
& =\sum_{i=0}^{2} \sum_{k} 2^{(3 k+i) r / q} \sup _{x_{3 k+i} \leqslant y \leqslant x_{3(k+1)+i}} U^{r}\left(y, x_{3 k+i}\right) V^{-r / p}(y) .
\end{aligned}
$$

Далее, из оценки (3.76) аналогичным разложением получаем

$$
\begin{gathered}
\sum_{k} 2^{3 k}\left(\sup _{x_{3 k+i} \leqslant y \leqslant x_{3(k+1)+i}} U^{p}\left(y, x_{3 k+i}\right) \int_{y}^{x_{3(k+1)+i}} h\right)^{q / p} \\
\ll C_{5}^{q}\left(\int_{0}^{\infty} h V\right)^{q / p}, \quad i=0,1,2
\end{gathered}
$$

а отсюда аналогично доказательству оценки $\mathbb{D} \ll C_{3}$ находим, что

$$
\sum_{k} 2^{3 k r / q} \sup _{x_{3 k+i} \leqslant y \leqslant x_{3(k+1)+i}} U^{r}\left(y, x_{3 k+i}\right) V^{-r / p}(y) \ll C_{5}^{r},
$$

и оценка $\mathbf{B}_{1} \ll C_{5}$ установлена. Теорема 3.12 доказана.

Симметричным вариантом предыдущей теоремы является следующее утверждение. Положим $\psi(x):=W_{*}^{-1}\left(4 W_{*}(x)\right)$, где $W_{*}^{-1}(t):=\inf \left\{s \geqslant 0: W_{*}(s)=t\right\}$.

Теорема 3.13. Пусть $0<q<p \leqslant 1$. Следующие условия эквивалентны:

$$
\begin{aligned}
& \left(\int_{0}^{\infty}\left(\int_{0}^{x} f u\right)^{q} w(x) d x\right)^{1 / q} \leqslant \bar{C}_{1}\left(\int_{0}^{\infty} f^{p} v\right)^{1 / p}, \quad f \in \mathfrak{M}^{\uparrow}, \\
& \left(\int_{0}^{\infty}\left(\int_{0}^{x} U^{p}(x, y) h(y) d y\right)^{q / p} w(x) d x\right)^{p / q} \leqslant \bar{C}_{2}^{p} \int_{0}^{\infty} h V_{*}, \quad h \in \mathfrak{M}^{+}, \\
& \left(\int_{0}^{\infty}\left(\sup _{0<y \leqslant x} U^{p}(x, y) \int_{0}^{y} h\right)^{q / p} w(x) d x\right)^{p / q} \leqslant \bar{C}_{3}^{p} \int_{0}^{\infty} h V_{*}, \quad h \in \mathfrak{M}^{+}, \\
& \left(\int_{0}^{\infty}\left(\sup _{0<y \leqslant x} U(x, y) f(y)\right)^{q} w(x) d x\right)^{1 / q} \leqslant \bar{C}_{4}\left(\int_{0}^{\infty} f^{p} v\right)^{1 / p}, \quad f \in \mathfrak{M}^{\uparrow}, \\
& \left(\int_{0}^{\infty}\left(\sup _{\psi(x) \leqslant y \leqslant x} U^{p}(x, y) \int_{0}^{y} h\right)^{q / p} w(x) d x\right)^{p / q} \leqslant \bar{C}_{5}^{p} \int_{0}^{\infty} h V_{*}, \quad h \in \mathfrak{M}^{+}, \\
& \left(\int_{0}^{\infty}\left(\sup _{\psi(x) \leqslant y \leqslant x} U(y, x) f(y)\right)^{q} w(x) d x\right)^{1 / q} \leqslant \bar{C}_{6}\left(\int_{0}^{\infty} f^{p} v\right)^{1 / p}, \quad f \in \mathfrak{M}^{\uparrow},
\end{aligned}
$$




$$
\begin{aligned}
& \left(\int_{0}^{\infty}\left[\left(\int_{\psi(x)}^{x} U^{p}(x, y) h(y) d y\right)^{q / p}+U^{q}(x, \psi(x))\left(\int_{0}^{\psi(x)} h\right)^{q / p}\right] w(x) d x\right)^{p / q} \\
& \quad \leqslant \bar{C}_{7}^{p} \int_{0}^{\infty} h V_{*}
\end{aligned}
$$

для всех $h \in \mathfrak{M}^{+}$,

$$
\begin{gathered}
\overline{\mathbb{B}}^{r}:=\sup _{\left\{x_{k}\right\}} \sum_{k \in \mathbb{Z}}\left(\int_{x_{k}}^{x_{k+1}} U^{q}\left(y, x_{k}\right) w(y) d y\right)^{r / q} V_{*}^{-r / p}\left(x_{k}\right)<\infty, \\
\overline{\mathbf{B}}_{1}+\overline{\mathbf{B}}_{2}<\infty
\end{gathered}
$$

где

$$
\begin{aligned}
& \overline{\mathbf{B}}_{1}^{r}:=\int_{0}^{\infty} \sup _{\psi(x) \leqslant y \leqslant x} U^{r}(x, y) V_{*}^{-r / p}(y) W_{*}^{r / p}(x) w(x) d x, \\
& \overline{\mathbf{B}}_{2}^{r}:=\int_{0}^{\infty}\left(\int_{x}^{\infty} w(y) U^{q}(y, \psi(y)) d y\right)^{r / p} V_{*}^{-r / p}(\varphi(x)) w(x) U^{q}(x, \psi(x)) d x .
\end{aligned}
$$

Более того,

$$
\bar{C}_{1} \approx \bar{C}_{2} \approx \bar{C}_{3}=\bar{C}_{4} \approx \bar{C}_{5} \approx \bar{C}_{6} \approx \bar{C}_{7} \approx \overline{\mathbb{B}} .
$$

ЗАмечАниЕ 3.2. Теорема 3.13 дополняет [44].

3.4. Дальнейшие результаты и комментарии. Сначала мы дадим полную характеризацию неравенства (3.34).

Теорема 3.14. Пусть $0<q, p<\infty u k(x, y) \geqslant 0-$ измеримое ядро. Тогда неравенство (3.34) с наилучшей константой $C_{1}$ выполняется для всех $f \in \mathfrak{M}^{\downarrow}$, если и только если:

(i) $0<p \leqslant 1, p \leqslant q<\infty$; в этом случае

$$
C_{1}=C_{2}:=\sup _{x \in(0, \infty)}\left(\int_{0}^{\infty} K^{q}(x, \min (x, y)) w(y) d y\right)^{1 / q} V^{-1 / p}(x)<\infty ;
$$

(ii) $q=1<p<\infty$; в этом случае $C_{1} \approx C_{3}$, где

$$
C_{3}:=\left(\int_{0}^{\infty}\left(\int_{x}^{\infty}\left(\int_{y}^{\infty} k(z, y) w(z) d z\right) V^{-1}(y) u(y) d y\right)^{p^{\prime}} v(x) d x\right)^{1 / p^{\prime}}<\infty .
$$

Если $k(x, y)$ - ядро Ойнарова, то

(iii) $0<q<p \leqslant 1$ u $k(x, y)$ непрерьвно; в этом случае

$$
C_{1} \approx C_{4}:=\left(\sup _{\left\{x_{k}\right\}} \sum_{k \in \mathbb{Z}}\left(\int_{x_{k}}^{x_{k+1}} K^{q}\left(y, x_{k}\right) w(y) d y\right)^{r / q} V^{-r / p}\left(x_{k}\right)\right)^{1 / r}<\infty ;
$$

(iv) $1<p \leqslant q<\infty$; в этом случае $C_{1} \approx C_{5}+C_{6}+C_{7}$, где

$$
\left.C_{4}:=\sup _{x \in(0, \infty)}\left(\int_{0}^{x} K^{q}(y, y)\right) w(y) d y\right)^{1 / q} V^{-1 / p}(x)<\infty,
$$




$$
\begin{aligned}
& C_{5}:=\sup _{x \in(0, \infty)} W_{*}^{1 / q}(x)\left(\int_{0}^{x} K^{p^{\prime}}(x, y) V^{-p^{\prime}}(y) v(y) d y\right)^{1 / p^{\prime}}<\infty \\
& C_{6}:=\sup _{x \in(0, \infty)}\left(\int_{x}^{\infty} k^{q}(y, x) w(y) d y\right)^{1 / q}\left(\int_{0}^{x} U^{p^{\prime}}(y) V^{-p^{\prime}}(y) v(y) d y\right)^{1 / p^{\prime}}<\infty
\end{aligned}
$$

(v) $1<q<p<\infty$; в этом случае $C_{1} \approx C_{8}+C_{9}+C_{10}$, где

$$
\begin{aligned}
C_{8}:= & \left(\int_{0}^{\infty}\left(\int_{0}^{x} K^{q}(y, y) w(y) d y\right)^{r / p} K^{q}(x, x) w(x) V^{-r / p}(x) d x\right)^{1 / r}<\infty, \\
C_{9}:= & \left(\int_{0}^{\infty}\left(\int_{x}^{\infty} k^{q}(y, x) w(y) d y\right)^{r / q}\right. \\
& \left.\times\left(\int_{0}^{x} U^{p^{\prime}}(y) V^{-p^{\prime}}(y) v(y) d y\right)^{r / p^{\prime}} U^{p^{\prime}}(x) V^{-p^{\prime}}(x) v(x) d x\right)^{1 / r}<\infty \\
C_{10}:= & \left(\int_{0}^{\infty} W_{*}^{r / p}(x) w(x)\left(\int_{0}^{x} K^{p^{\prime}}(x, y) V^{-p^{\prime}}(y) v(y) d y\right)^{r / p^{\prime}} d x\right)^{1 / r}<\infty ;
\end{aligned}
$$

(vi) $0<q<1<p<\infty$; в этом случае $C_{1} \approx C_{8}+C_{11}+C_{12}<\infty$, где

$$
\begin{aligned}
C_{11}:= & \left(\sup _{\left\{x_{k}\right\}} \sum_{k \in \mathbb{Z}}\left(\int_{x_{k}}^{x_{k+1}} w\right)^{r / q}\left(\int_{x_{k-1}}^{x_{k}} K^{p^{\prime}}\left(x_{k}, y\right) V^{-p^{\prime}}(y) v(y) d y\right)^{r / p^{\prime}}\right)^{1 / r}<\infty, \\
C_{12}:= & \left(\sup _{\left\{x_{k}\right\}} \sum_{k \in \mathbb{Z}}\left(\int_{x_{k}}^{x_{k+1}} k^{q}\left(y, x_{k}\right)^{q} w(y) d y\right)^{r / q}\right. \\
& \left.\times\left(\int_{x_{k-1}}^{x_{k}} U^{p^{\prime}}(y) V^{-p^{\prime}}(y) v(y) d y\right)^{r / p^{\prime}}\right)^{1 / r}<\infty .
\end{aligned}
$$

ДокАзАтельство. Утверждение (i) следует из [66], а (ii) - из теоремы 2.5, (iii) - это теорема 3.9. Части (iv) и (v) получены в теореме 7, [78], а для доказательства (vi) следует применить теоремы 3.1 и 1.3. С помощью теоремы 1.4 можно заменить константы $C_{11}$ и $C_{12}$ на интегральные. Теорема 3.14 доказана.

Для предельных случаев $p=\infty, q=\infty$ мы имеем следующие результаты.

Теорема 3.15. Пусть $0<p<\infty u k(x, y) \geqslant 0-$ измеримое ядро. Тогда неравенство

$$
\operatorname{essip}_{x \in(0, \infty)}\left(\int_{0}^{x} k(x, y) f(y) u(y) d y\right) w(x) \leqslant C\left(\int_{0}^{\infty} f^{p} v\right)^{1 / p}
$$

верно для всех $f \in \mathfrak{M}^{\downarrow}$, если и толъко если:

(i) $0<p \leqslant 1$; в этом случае

$$
C=\sup _{x \in(0, \infty)}\left(\operatorname{esssup}_{y \in(0, \infty)} K(x, \min (x, y)) w(y)\right) V^{-1 / p}(x)<\infty ;
$$

(ii) $1<p<\infty$; в этом случае

$$
C=\operatorname{esssup}_{s \in(0, \infty)} w(s)\left(\int_{0}^{s}\left(\int_{t}^{s} k(s, y) u(y) V^{-1}(y) d y\right)^{p^{\prime}} v(t) d t\right)^{1 / p^{\prime}}<\infty .
$$


Теорема 3.16. Пусть $\|\cdot\|_{X}-$ произвольная квазинорма на $\mathfrak{M}^{+} u k(x, y) \geqslant 0-$ измеримое ядро на $\{(x, y): x \geqslant y \geqslant 0\}$. Тогда неравенство

$$
\left\|\int_{0}^{x} k(x, y) f(y) d y\right\|_{X} \leqslant C\|f v\|_{\infty}
$$

выполняется для всех $f \in \mathfrak{M}^{\downarrow}$, если и только если

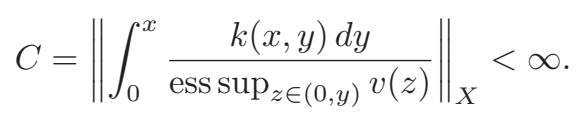

Для формулировки следующей теоремы нам потребуются дополнительные обозначения:

$$
\begin{aligned}
V_{k}(x) & :=\int_{x}^{\infty} k(y, x) \frac{u(y)}{V^{2}(y)} d y, \\
V_{1}(x) & :=\int_{x}^{\infty} \frac{u(y)}{V^{2}(y)} d y .
\end{aligned}
$$

Теорема 3.17. Пусть $0<q, p<\infty u k(x, y) \geqslant 0-я$ неравенство (3.46) выполняется для всех $f \in \mathfrak{M}^{\downarrow}$ с наилучшей константой $C_{1}$, если и только если:

(i) $0<p \leqslant 1, p \leqslant q<\infty$; в этом случае

$$
C_{1}=C_{2}:=\sup _{x \in(0, \infty)}\left(\int_{0}^{x} U_{k}^{q}(x, y) w(y) d y\right)^{1 / q} V^{-1 / p}(x)<\infty
$$

(ii) $0<q<p \leqslant 1$; в этом случае

$$
C_{1} \approx C_{3}:=\left(\sup _{\left\{x_{k}\right\}} \sum_{k \in \mathbb{Z}}\left(\int_{x_{k}}^{x_{k+1}} U_{k}^{q}\left(x_{k+1}, y\right) w(y) d y\right)^{r / q} V^{-r / p}\left(x_{k+1}\right)\right)^{1 / r}<\infty
$$

(iii) $1<p \leqslant q<\infty$; в этом случае $C_{1} \approx A_{1}+A_{2}+A_{3}$, где

$$
\begin{aligned}
A_{1}:= & \sup _{x \in(0, \infty)}\left(\int_{x}^{\infty} V_{k}^{q} w\right)^{1 / q}[V(x)]^{1+1 / p^{\prime}}, \\
A_{2}:= & \sup _{x \in(0, \infty)} W^{1 / q}(x)\left(\int_{x}^{\infty}\left(V V_{k}\right)^{p^{\prime}} v\right)^{1 / p^{\prime}}, \\
A_{3}:= & \sup _{x \in(0, \infty)}\left(\int_{0}^{x} k^{q}(x, s) w(s) d s\right)^{1 / q}\left(\int_{x}^{\infty}\left(V V_{1}\right)^{p^{\prime}} v\right)^{1 / p^{\prime}} \\
& \quad+\sup _{x \in(0, \infty)} W^{1 / q}(x)\left(\int_{x}^{\infty} k^{p^{\prime}}(s, x)\left(V V_{1}\right)^{p^{\prime}}(s) v(s) d s\right)^{1 / p^{\prime}} ;
\end{aligned}
$$

(iv) $1<q<p<\infty$; в этом случае $C_{1} \approx B_{1}+B_{2}+B_{3}$, где

$$
B_{1}:=\left(\int_{0}^{\infty}\left(\int_{x}^{\infty} V_{k}^{q} w\right)^{r / p}\left(\int_{0}^{x} V^{p^{\prime}} v\right)^{r / p^{\prime}} V_{k}^{q}(x) w(x) d x\right)^{1 / r},
$$




$$
\begin{aligned}
B_{2}:= & \left(\int_{0}^{\infty} W^{r / p}(x)\left(\int_{x}^{\infty}\left(V V_{k}\right)^{p^{\prime}} v\right)^{r / p^{\prime}} w(x) d x\right)^{1 / r} \\
B_{3}:= & \left(\int_{0}^{\infty}\left(\int_{0}^{x} k^{q}(x, s) w(s) d s\right)^{r / q}\left(\int_{x}^{\infty}\left(V V_{1}\right)^{p^{\prime}} v\right)^{r / q^{\prime}}\left(V(x) V_{1}(x)\right)^{p^{\prime}} v(x) d x\right)^{1 / r} \\
& +\left(\int_{0}^{\infty} W^{r / p}(x)\left(\int_{x}^{\infty} k^{p^{\prime}}(s, x)\left(V(s) V_{1}(s)\right)^{p^{\prime}} v(s) d s\right)^{r / q^{\prime}} w(x) d x\right)^{1 / r}
\end{aligned}
$$

(v) $q=1 \leqslant p<\infty$; в этом случае $C_{1}=C_{7}$, где

$$
C_{7}:=\left(\int_{0}^{\infty} V^{p^{\prime}}(x) v(x) d x\left(\int_{x}^{\infty} \frac{u(y) d y}{V^{2}(y)} \int_{0}^{y} k(y, s) w(s) d s\right)^{p^{\prime}}\right)^{1 / p^{\prime}},
$$

если $p>1, u$

$$
C_{7}:=\sup _{x \in(0, \infty)} V(x)\left(\int_{x}^{\infty} \frac{u(y) d y}{V^{2}(y)} \int_{0}^{y} k(y, s) w(s) d s\right)
$$

если $p=1$;

(vi) $0<q<1<p<\infty$; в этом случае $C_{1} \approx B_{1}+B_{2}+B_{4}$, где

$$
\begin{aligned}
B_{4}:= & \sup _{x_{k}}\left(\sum_{k}\left(\int_{x_{k}}^{x_{k+1}} w\right)^{r / q}\left(\int_{x_{k-1}}^{x_{k}} k^{p^{\prime}}\left(x_{k}, s\right)\left(V(s) V_{1}(s)\right)^{p^{\prime}} v(s) d s\right)^{r / p^{\prime}}\right)^{1 / r} \\
& +\sup _{x_{k}}\left(\sum_{k}\left(\int_{x_{k}}^{x_{k+1}} k^{p^{\prime}}\left(s, x_{k}\right) w(s) d s\right)^{r / q}\right. \\
& \left.\times\left(\int_{x_{k-1}}^{x_{k}}\left(V(s) V_{1}(s)\right)^{p^{\prime}} v(s) d s\right)^{r / p^{\prime}}\right)^{1 / r} .
\end{aligned}
$$

ДокАЗАТЕЛЬство. Часть (i) следует из [66], а (ii) - это теорема 3.11. Применяя теорему 3.1 при $q=1 \leqslant p<\infty$, мы сводим (3.46) к неравенству

$$
\int_{0}^{\infty} h(s) V(s)\left(\int_{s}^{\infty} \frac{u(y)}{V^{2}(y)}\left(\int_{0}^{y} k(y, x) w(x) d x\right) d y\right) \leqslant C_{1}\left(\int_{0}^{\infty} h^{p} v^{1-p}\right)^{1 / p},
$$

$h \in \mathfrak{M}^{+}$. Тогда (v) следует по теореме 1.1 . Из разложения

$$
\begin{aligned}
\int_{x}^{\infty} & k(y, x) \frac{u(y)}{V^{2}(y)} \int_{0}^{y} h(z) V(z) d z d y \\
= & \int_{0}^{x} h(z) V(z) d z \int_{x}^{\infty} k(y, x) \frac{u(y)}{V^{2}(y)} d y+\int_{x}^{\infty} k(y, x) \frac{u(y)}{V^{2}(y)} \int_{x}^{y} h(z) V(z) d z d y \\
= & \int_{0}^{x} h(z) V(z) d z \int_{x}^{\infty} k(y, x) \frac{u(y)}{V^{2}(y)} d y+\int_{x}^{\infty} h(z) V(z) \int_{z}^{\infty} k(y, x) \frac{u(y)}{V^{2}(y)} d y d z \\
\approx & \int_{0}^{x} h(z) V(z) d z \int_{x}^{\infty} k(y, x) \frac{u(y)}{V^{2}(y)} d y+\int_{x}^{\infty} h(z) V(z) \int_{z}^{\infty} k(y, z) \frac{u(y)}{V^{2}(y)} d y d z \\
& \quad+\int_{x}^{\infty} k(z, x) h(z) V(z) \int_{z}^{\infty} \frac{u(y)}{V^{2}(y)} d y d z
\end{aligned}
$$


вытекает, что для всех остальных случаев по теореме 3.1 неравенство (3.46) сводится к характеризации следующих трех неравенств на конусе $\mathfrak{M}^{+}$:

$$
\begin{gathered}
\left(\int_{0}^{\infty}\left(V_{k}(x) \int_{0}^{x} h V\right)^{q} w(x) d x\right)^{1 / q} \leqslant C_{4,1}\left(\int_{0}^{\infty} h^{p} v^{1-p}\right)^{1 / p} \\
\left(\int_{0}^{\infty}\left(\int_{x}^{\infty} h V V_{k}\right)^{q} w(x) d x\right)^{1 / q} \leqslant C_{4,2}\left(\int_{0}^{\infty} h^{p} v^{1-p}\right)^{1 / p}, \\
\left(\int_{0}^{\infty}\left(\int_{x}^{\infty} k(s, x) V(s) V_{1}(s) h(s) d s\right)^{q} w(x) d x\right)^{1 / q} \leqslant C_{4,3}\left(\int_{0}^{\infty} h^{p} v^{1-p}\right)^{1 / p},
\end{gathered}
$$

где $V_{k}$ и $V_{1}$ определены формулами (3.81) и (3.82) соответственно. Применяя теорему 1.2 и результаты работы [92], мы находим, что $C_{4,1} \approx A_{1}$ для $1<p \leqslant$ $q<\infty$ и $C_{4,1} \approx B_{1}$ для $0<q<p<\infty, p>1$ соответственно. Аналогично, $C_{4,2} \approx A_{2}$ для $1<p \leqslant q<\infty$ и $C_{4,2} \approx B_{2}$ для $0<q<p<\infty, p>1$. Используя теорему 1.2 , мы получаем, что $C_{4,3} \approx A_{3}$ при $1<p \leqslant q<\infty, C_{4,3} \approx B_{3}$ при $1<q<p<\infty$ и, по теореме $1.3, C_{4,3} \approx B_{4}$ при $0<q<1<p<\infty$. Теорема 3.17 доказана.

В частном случае $k(y, x)=1$ дополнительно к теореме 3.12 мы имеем полностью интегральную характеризацию неравенства (3.57).

СлеДСтвие 3.1. Пусть $0<q, p<\infty$. Тогда неравенство (3.57) с наилучшей константой $C_{1}$ верно для всех $f \in \mathfrak{M}^{\downarrow}$, если и только если:

(i) $0<q<p=1$; в этом случае $C_{1} \approx B_{1,0}+B_{2,0}$, где

$$
\begin{aligned}
& B_{1,0}:=\left(\int_{0}^{\infty} V^{q /(1-q)}(x)\left(\int_{x}^{\infty} V_{1}^{q} w\right)^{q /(1-q)} V_{1}^{q}(x) w(x) d x\right)^{(1-q) / q}, \\
& B_{2,0}:=\left(\int_{0}^{\infty}\left[\sup _{t \in(x, \infty)} V(t) V_{1}(t)\right]^{q /(1-q)} W^{q /(1-q)}(x) w(x) d x\right)^{(1-q) / q}
\end{aligned}
$$

(ii) $1<p \leqslant q<\infty$; в этом случае $C_{1} \approx A_{1,0}+A_{2,0}$, где

$$
\begin{aligned}
& A_{1,0}:=\sup _{x \in(0, \infty)}\left(\int_{x}^{\infty} V_{1}^{q} w\right)^{1 / q}[V(x)]^{1+1 / p^{\prime}} \\
& A_{2,0}:=\sup _{x \in(0, \infty)} W^{1 / q}(x)\left(\int_{x}^{\infty}\left(V V_{1}\right)^{p^{\prime}} v\right)^{1 / p^{\prime}} ;
\end{aligned}
$$

(iii) $1<q<p<\infty$; в этом случае $C_{1} \approx \mathbf{B}_{1,0}+\mathbf{B}_{2,0}$, где

$$
\begin{aligned}
\mathbf{B}_{1,0} & :=\left(\int_{0}^{\infty}\left(\int_{x}^{\infty} V_{1}^{q} w\right)^{r / p}[V(x)]^{r+r / p^{\prime}} V_{1}^{q}(x) w(x) d x\right)^{1 / r}, \\
\mathbf{B}_{2,0} & :=\left(\int_{0}^{\infty} W^{r / p}(x)\left(\int_{x}^{\infty}\left(V V_{1}\right)^{p^{\prime}} v\right)^{r / p^{\prime}} w(x) d x\right)^{1 / r} ;
\end{aligned}
$$


(iv) $q=1 \leqslant p<\infty$; в этом случае $C_{1}=C_{7,0}$, где

$$
C_{7,0}:=\left(\int_{0}^{\infty} V^{p^{\prime}}(x) v(x) d x\left(\int_{x}^{\infty} \frac{W(y) u(y) d y}{V^{2}(y)}\right)^{p^{\prime}}\right)^{1 / p^{\prime}},
$$

если $p>1$, и с объчной модификацией для $p=1$;

(v) $0<q<1<p<\infty$; в этом случае $C_{1} \approx \mathbf{B}_{1,0}+\mathbf{B}_{2,0}$.

Для формулировки результатов, двойственных к двум предыдущим утверждениям, введем следующие обозначения:

$$
\begin{gathered}
V_{*}(t):=\int_{t}^{\infty} v, \quad W_{*}(t):=\int_{t}^{\infty} w, \quad U_{k}^{*}(x, y):=\int_{y}^{x} k(x, y) u(z) d z, \\
V_{k}^{*}(x):=\int_{0}^{x} k(x, y) \frac{u(y)}{V_{*}^{2}(y)} d y, \quad V_{1}^{*}(x):=\int_{0}^{x} \frac{u(y)}{V_{*}^{2}(y)} d y .
\end{gathered}
$$

Tеорема 3.18. Пусть $0<q, p<\infty u k(x, y) \geqslant 0-я$ яро Ойнарова. Тогда неравенство

$$
\left(\int_{0}^{\infty}\left(\int_{0}^{x} k(x, y) f(y) u(y) d y\right)^{q} w(x) d x\right)^{1 / q} \leqslant \mathscr{C}_{1}\left(\int_{0}^{\infty} f^{p} v\right)^{1 / p}
$$

с наилучшей константой $\mathscr{C}_{1}$ имеет место для всех $f \in \mathfrak{M}^{\uparrow}$, если и только если:

(i) $0<p \leqslant 1, p \leqslant q<\infty$; в этом случае

$$
\mathscr{C}_{1}=\mathscr{C}_{2}:=\sup _{x \in(0, \infty)}\left(\int_{x}^{\infty}\left[U_{k}^{*}(y, x)\right]^{q} w(y) d y\right)^{1 / q} V_{*}^{-1 / p}(x)<\infty ;
$$

(ii) $0<q<p \leqslant 1$; в этом случае

$$
\mathscr{C}_{1} \approx \mathscr{C}_{3}:=\left(\sup _{\left\{x_{k}\right\}} \sum_{k \in \mathbb{Z}}\left(\int_{x_{k}}^{x_{k+1}}\left[U_{k}^{*}\left(\left(y, x_{k}\right)\right]^{q} w(y) d y\right)^{r / q} V_{*}^{-r / p}\left(x_{k}\right)\right)^{1 / r}<\infty\right.
$$

(iii) $1<p \leqslant q<\infty$; в этом случае $\mathscr{C}_{1} \approx \mathscr{A}_{1}+\mathscr{A}_{2}+\mathscr{A}_{3}$, где

$$
\begin{aligned}
\mathscr{A}_{1}:= & \sup _{x \in(0, \infty)}\left(\int_{0}^{x}\left[V_{k}^{*}\right]^{q} w\right)^{1 / q}\left[V_{*}(x)\right]^{1+1 / p^{\prime}}, \\
\mathscr{A}_{2}:= & \sup _{x \in(0, \infty)} W_{*}^{1 / q}(x)\left(\int_{0}^{x}\left(V_{*} V_{k}^{*}\right)^{p^{\prime}} v\right)^{1 / p^{\prime}}, \\
\mathscr{A}_{3}:= & \sup _{x \in(0, \infty)}\left(\int_{x}^{\infty} k^{q}(s, x) w(s) d s\right)^{1 / q}\left(\int_{0}^{x}\left(V_{*} V_{1}^{*}\right)^{p^{\prime}} v\right)^{1 / p^{\prime}} \\
& +\sup _{x \in(0, \infty)} W_{*}^{1 / q}(x)\left(\int_{0}^{x} k^{p^{\prime}}(x, s)\left(V_{*} V_{1}^{*}\right)^{p^{\prime}}(s) v(s) d s\right)^{1 / p^{\prime}} ;
\end{aligned}
$$


(iv) $1<q<p<\infty$; в этом случае $\mathscr{C}_{1} \approx \mathscr{B}_{1}+\mathscr{B}_{2}+\mathscr{B}_{3}$, где

$$
\begin{aligned}
\mathscr{B}_{1}:= & \left(\int_{0}^{\infty}\left(\int_{0}^{x}\left[V_{k}^{*}\right]^{q} w\right)^{r / p}\left[V_{*}(x)\right]^{r+r / p^{\prime}}\left[V_{k}^{*}\right]^{q}(x) w(x) d x\right)^{1 / r}, \\
\mathscr{B}_{2}:= & \left(\int_{0}^{\infty} W_{*}^{r / p}(x)\left(\int_{0}^{x}\left(V_{*} V_{k}^{*}\right)^{p^{\prime}} v\right)^{r / p^{\prime}} w(x) d x\right)^{1 / r}, \\
\mathscr{B}_{3}:= & \left(\int_{0}^{\infty}\left(\int_{x}^{\infty} k^{q}(s, x) w(s) d s\right)^{r / q}\left(\int_{0}^{x}\left(V_{*} V_{1}^{*}\right)^{p^{\prime}} v\right)^{r / q^{\prime}}\left(V_{*}(x) V_{1}^{*}(x)\right)^{p^{\prime}} v(x) d x\right)^{1 / r} \\
& +\left(\int_{0}^{\infty} W_{*}^{r / p}(x)\left(\int_{0}^{x} k^{p^{\prime}}(x, s)\left(V_{*}(s) V_{1}^{*}(s)\right)^{p^{\prime}} v(s) d s\right)^{r / q^{\prime}} w(x) d x\right)^{1 / r} ;
\end{aligned}
$$

(v) $q=1 \leqslant p<\infty ;$ в этом случае $\mathscr{C}_{1}=\mathscr{C}_{7}$, где

$$
\mathscr{C}_{7}:=\left(\int_{0}^{\infty} V_{*}^{p^{\prime}}(x) v(x) d x\left(\int_{0}^{x} \frac{u(y) d y}{V_{*}^{2}(y)} \int_{y}^{\infty} k(s, y) w(s) d s\right)^{p^{\prime}}\right)^{1 / p^{\prime}},
$$

если $p>1, u$

$$
\mathscr{C}_{7}:=\sup _{x \in(0, \infty)} V_{*}(x)\left(\int_{0}^{x} \frac{u(y) d y}{V_{*}^{2}(y)} \int_{y}^{\infty} k(s, y) w(s) d s\right)
$$

при $p=1$;

(vi) $0<q<1<p<\infty$; в этом случае $\mathscr{C}_{1} \approx \mathscr{B}_{1}+\mathscr{B}_{2}+\mathscr{B}_{4}$, где

$$
\begin{aligned}
\mathscr{B}_{4}:= & \sup _{x_{k}}\left(\sum_{k}\left(\int_{x_{k-1}}^{x_{k}} w\right)^{r / q}\left(\int_{x_{k}}^{x_{k+1}} k^{p^{\prime}}\left(s, x_{k}\right)\left(V_{*}(s) V_{1}^{*}(s)\right)^{p^{\prime}} v(s) d s\right)^{r / p^{\prime}}\right)^{1 / r} \\
& +\sup _{x_{k}}\left(\sum_{k}\left(\int_{x_{k-1}}^{x_{k}} k^{p^{\prime}}\left(x_{k}, s\right) w(s) d s\right)^{r / q}\left(\int_{x_{k}}^{x_{k+1}}\left(V_{*}(s) V_{1}^{*}(s)\right)^{p^{\prime}} v(s) d s\right)^{r / p^{\prime}}\right)^{1 / r} .
\end{aligned}
$$

При $k(y, x)=1$ в дополнение к теореме 3.13 мы имеем полностью интегральную характеризацию неравенства (3.79).

СЛЕДСТвиЕ 3.2. Пусть $0<q, p<\infty$. Тогда неравенство

$$
\left(\int_{0}^{\infty}\left(\int_{0}^{x} f(y) u(y) d y\right)^{q} w(x) d x\right)^{1 / q} \leqslant C_{1,0}^{*}\left(\int_{0}^{\infty} f^{p} v\right)^{1 / p}
$$

с наилучшей константой $C_{1,0}^{*}$ выполнено для всех $f \in \mathfrak{M}^{\uparrow}$, если и только если:

(i) $0<q<p=1$; в этом случае $C_{1,0}^{*} \approx B_{1,0}^{*}+B_{2,0}^{*}$, где

$$
\begin{aligned}
& B_{1,0}^{*}:=\left(\int_{0}^{\infty} V_{*}^{q /(1-q)}(x)\left(\int_{0}^{x}\left[V_{1}^{*}\right]^{q} w\right)^{q /(1-q)}\left[V_{1}^{*}\right]^{q}(x) w(x) d x\right)^{(1-q) / q}, \\
& B_{2,0}^{*}:=\left(\int_{0}^{\infty}\left[\sup _{t \in(0, x)} V_{*}(t) V_{1}^{*}(t)\right]^{q /(1-q)} W_{*}^{q /(1-q)}(x) w(x) d x\right)^{(1-q) / q}
\end{aligned}
$$


(ii) $1<p \leqslant q<\infty$; в этом случае $C_{1,0}^{*} \approx A_{1,0}^{*}+A_{2,0}^{*}$, где

$$
\begin{aligned}
& A_{1,0}^{*}:=\sup _{x \in(0, \infty)}\left(\int_{0}^{x}\left[V_{1}^{*}\right]^{q} w\right)^{1 / q}\left[V_{*}(x)\right]^{1+1 / p^{\prime}}, \\
& A_{2,0}^{*}:=\sup _{x \in(0, \infty)} W_{*}^{1 / q}(x)\left(\int_{0}^{x}\left(V_{*} V_{1}^{*}\right)^{p^{\prime}} v\right)^{1 / p^{\prime}} ;
\end{aligned}
$$

(iii) $1<q<p<\infty$; в этом случае $C_{1,0}^{*} \approx \mathbf{B}_{1,0}^{*}+\mathbf{B}_{2,0}^{*}$, где

$$
\begin{aligned}
\mathbf{B}_{1,0}^{*} & :=\left(\int_{0}^{\infty}\left(\int_{0}^{x}\left[V_{1}^{*}\right]^{q} w\right)^{r / p}\left[V_{*}(x)\right]^{r+r / p^{\prime}}\left[V_{1}^{*}\right]^{q}(x) w(x) d x\right)^{1 / r}, \\
\mathbf{B}_{2,0}^{*} & :=\left(\int_{0}^{\infty} W_{*}^{r / p}(x)\left(\int_{0}^{x}\left(V_{*} V_{1}^{*}\right)^{p^{\prime}} v\right)^{r / p^{\prime}} w(x) d x\right)^{1 / r} ;
\end{aligned}
$$

(iv) $q=1 \leqslant p<\infty ;$ в этом случае $C_{1,0}^{*}=C_{7,0}^{*}$, где

$$
C_{7,0}^{*}:=\left(\int_{0}^{\infty} V_{*}^{p^{\prime}}(x) v(x) d x\left(\int_{0}^{x} \frac{W_{*}(y) u(y) d y}{V_{*}^{2}(y)}\right)^{p^{\prime}}\right)^{1 / p^{\prime}},
$$

если $p>1$, с обычной модификацией для $p=1$;

(v) $0<q<1<p<\infty$; в этом случае $C_{1,0}^{*} \approx \mathbf{B}_{1,0}^{*}+\mathbf{B}_{2,0}^{*}$.

Пусть $k(x, y) \geqslant 0$ - ядро Ойнарова. Одним из любопытных приложений редукционных теорем (в обратном направлении!) является нахождение интегрального критерия для неравенств

$$
\left(\int_{0}^{\infty}\left(\int_{0}^{x} k(x, y) h(y) d y\right)^{q} w(x) d x\right)^{1 / q} \leqslant C\left(\int_{0}^{\infty} h^{p} \sigma\right)^{1 / p}, \quad h \in \mathfrak{M}^{+},
$$

и

$$
\left(\int_{0}^{\infty}\left(\int_{x}^{\infty} k(y, x) h(y) d y\right)^{q} w(x) d x\right)^{1 / q} \leqslant C\left(\int_{0}^{\infty} h^{p} \sigma\right)^{1 / p}, \quad h \in \mathfrak{M}^{+},
$$

при $0<q<1 \leqslant p<\infty$ в случае

$$
k(x, y)=\int_{y}^{x} u(s) d s=: U(x, y) .
$$

При этом ответ получается проще, чем при применении теорем 1.3 и 1.4. Мы продемонстрируем решение для (3.88), для (3.87) рассуждения аналогичны. Введем обозначения

$$
\begin{gathered}
S(x):=\int_{x}^{\infty} \sigma^{1-p^{\prime}}, \quad 1<p<\infty, \quad v_{\sigma}(x):=[S(x)]^{-p}[\sigma(x)]^{1-p^{\prime}}, \\
V_{\sigma}(x):=\int_{0}^{x} v_{\sigma}, \quad V_{1, \sigma}(x):=\int_{x}^{\infty} S^{2(p-1)} u .
\end{gathered}
$$


СлеДСтвиЕ 3.3. Пусть $0<q<1 \leqslant p<\infty$. Тогда неравенство

$$
\left(\int_{0}^{\infty}\left(\int_{x}^{\infty} U(y, x) h(y) d y\right)^{q} w(x) d x\right)^{1 / q} \leqslant C\left(\int_{0}^{\infty} h^{p} \sigma\right)^{1 / p}, \quad h \in \mathfrak{M}^{+}
$$

верно, если и только если:

(i) $0<q<1<p<\infty$; в этом случае $C \approx B_{1,1}+B_{2,1}$, где

$$
\begin{aligned}
B_{1,1} & :=\left(\int_{0}^{\infty}\left(\int_{x}^{\infty}\left[V_{1, \sigma}\right]^{q} w\right)^{r / p}[S(x)]^{(1-2 p) r / p^{\prime}}\left[V_{1, \sigma}\right]^{q}(x) w(x) d x\right)^{1 / r}, \\
B_{2,1} & :=\left(\int_{0}^{\infty} W^{r / p}(x)\left(\int_{0}^{x}\left(V_{\sigma} V_{1, \sigma}\right)^{p^{\prime}} v_{\sigma}\right)^{r / p^{\prime}} w(x) d x\right)^{1 / r}
\end{aligned}
$$

(ii) $0<q<1=p$; в этом случае $C \approx B_{1,2}+B_{2,2}$, где

$$
\begin{aligned}
B_{1,2} & :=\left(\int_{0}^{\infty} V_{\sigma}^{q /(1-q)}(x)\left(\int_{0}^{x}\left[V_{1, \sigma}\right]^{q} w\right)^{q /(1-q)}\left[V_{1, \sigma}\right]^{q}(x) w(x) d x\right)^{(1-q) / q}, \\
B_{2,2} & :=\left(\int_{0}^{\infty}\left[\sup _{t \in(x, \infty)} V_{\sigma}(t) V_{1, \sigma}(t)\right]^{q /(1-q)} W^{q /(1-q)}(x) w(x) d x\right)^{(1-q) / q} .
\end{aligned}
$$

ДоказАтельство. Начнем с утверждения (i). Заметим, что

$$
V_{\sigma}(x)=\int_{0}^{x}[S(y)]^{-p}[\sigma(y)]^{1-p^{\prime}} d y \approx\left([S(x)]^{1-p}-[S(0)]^{1-p}\right)
$$

и

$$
\left[V_{\sigma}(x)\right]^{p}\left[v_{\sigma}(x)\right]^{1-p}=\left([S(x)]^{1-p}-[S(0)]^{1-p}\right)^{p}[S(x)]^{p(p-1)} \sigma(x) \leqslant \sigma(x) .
$$

По теореме 3.1 неравенство

$$
\left(\int_{0}^{\infty}\left(\int_{x}^{\infty} U(y, x) h(y) d y\right)^{q} w(x) d x\right)^{1 / q} \leqslant C_{1}\left(\int_{0}^{\infty} h^{p} V_{\sigma}^{p} v_{\sigma}^{1-p}\right)^{1 / p}
$$

эквивалентно неравенству

$$
\left(\int_{0}^{\infty}\left(\int_{x}^{\infty} u(y) f(y) d y\right)^{q} w(x) d x\right)^{1 / q} \leqslant C_{2}\left(\int_{0}^{\infty} f^{p} v_{\sigma}\right)^{1 / p}, \quad f \in \mathfrak{M}^{\downarrow},
$$

характеризованному следствием 3.1 так, что

$$
\begin{array}{ll}
C_{2} \approx B_{1,1}+B_{2,1}, & 0<q<1<p<\infty \\
C_{2} \approx B_{1,2}+B_{2,2}, & 0<q<1=p .
\end{array}
$$

Таким образом,

$$
\begin{array}{ll}
C \ll B_{1,1}+B_{2,1}, & 0<q<1<p<\infty ; \\
C \ll B_{1,2}+B_{2,2}, & 0<q<1=p .
\end{array}
$$

Для доказательства обратных неравенств предположим, что (3.89) верно с константой $C \in(0, \infty)$. Тогда $(3.89)$ с той же константой $C$ также верно для $\sigma_{\varepsilon}:=$ 
$\sigma+\varepsilon, w_{\delta}:=w \chi_{(\delta, 1 / \delta)}$ вместо $\sigma$ и $w$ соответственно, где $\varepsilon>0$ и $\delta \in(0,1)$. Для этих весовых функций $S(0)=\infty$ и, следовательно, $B_{i, j}^{\varepsilon, \delta} \ll C$, где $B_{i, j}^{\varepsilon, \delta}<\infty$ - это константа $B$ с модифицированными весами. По теореме Лебега о мажорируемой сходимости получаем $\lim _{\delta \rightarrow 0}\left(\lim _{\varepsilon \rightarrow 0} B_{i, j}^{\varepsilon, \delta}\right)=B_{i, j}$, откуда следует обратное неравенство.

Случай (ii) доказывается аналогично.

\section{Список литературы}

[1] M. Ariño, B. Muckenhoupt, "Maximal functions on classical Lorentz spaces and Hardy's inequality with weights for nonincreasing functions", Trans. Amer. Math. Soc., 320:2 (1990), 727-735.

[2] G. Bennett, K.-G. Grosse-Erdmann, "Weighted Hardy inequalities for decreasing sequences and functions", Math. Ann., 334:3 (2006), 489-531.

[3] Е. И. Бережной, "Точные оценки операторов на конусах в идеальных пространствах", Исследования по теории дифферениируемых функиий многих переменных и ее приложениям. Часть 16, Тр. МИАН, 204, Наука, М., 1993, 3-34; англ. пер.: E. I. Berezhnoi, "Sharp estimates for operators on cones in ideal spaces", Proc. Steklov Inst. Math., 204:3 (1994), 1-25.

[4] S. Bloom, R. Kerman, "Weighted norm inequalities for operators of Hardy type", Proc. Amer. Math. Soc., 113:1 (1991), 135-141.

[5] M. Sh. Braverman, "On a class of operators", J. London Math. Soc. (2), 47:1 (1993), 119-128.

[6] В. И. Буренков, М. Л. Гольдман, "Вычисление нормы положительного оператора на конусе монотонных функций", Теория функций и дифференииальные уравнения, Сборник статей. $\mathrm{K}$ девяностолетию со дня рождения академика Сергея Михайловича Никольского, Тр. МИАН, 210, Наука, М., 1995, 65-89; англ. пер.: V.I. Burenkov, M. L. Goldman, "Calculation of the norm of a positive operator on the cone of monotone functions", Proc. Steklov Inst. Math., 210 (1995), 47-65.

[7] M. J. Carro, A. Gogatishvili, J. Martin, L. Pick, "Function properties of rearrangement invariant spaces defined in terms of oscillations", J. Funct. Anal., 229:2 (2005), 375-404.

[8] M. Carro, A. Gogatishvili, J. Martin, L. Pick, "Weighted inequalities involving two Hardy operators with applications to embeddings of function spaces", J. Operator Theory, 59:2 (2008), 309-332.

[9] M. Carro, L. Pick, J. Soria, V. D. Stepanov, "On embeddings between clasical Lorentz spaces", Math. Inequal. Appl., 4:3 (2001), 397-428.

[10] M. J. Carro, J. A. Raposo, J. Soria, Recent developments in the theory of Lorentz spaces and weighted inequalities, Mem. Amer. Math. Soc., 187, № 877, 2007, xii+128 pp.

[11] M. Carro, J. Soria, "Weighted Lorentz spaces and the Hardy operator", J. Funct. Anal., 112:2 (1993), 480-494.

[12] M. Carro, J. Soria, "Boundedness of some integral operators", Canad. J. Math., 45 (1993), 1155-1166.

[13] M. Carro, J. Soria, "The Hardy-Littlewood maximal function and weighted Lorentz spaces", J. London Math. Soc. (2), 55:1 (1997), 146-158.

[14] P. Drábek, H. P. Heinig, A. Kufner, "Weighted modular inequalities for monotone functions", J. Inequal. Appl., 1:2 (1997), 183-197.

[15] D. E. Edmunds, W.D. Evans, Hardy operators, function spaces and embeddings, Springer Monogr. Math., Springer-Verlag, Berlin, 2004, xii+326 pp. 
[16] D. E. Edmunds, V.D. Stepanov, "The measure of non-compactness and approximation numbers of certain Volterra integral operators", Math. Ann., 298:1 (1994), 41-66.

[17] D. E. Edmunds, V. D. Stepanov, "On the singular numbers of certain Volterra integral operators", J. Funct. Anal., 134:1 (1995), 222-246.

[18] W. D. Evans, A. Gogatishvili, B. Opic, "The reverse Hardy inequality with measures", Math. Inequal. Appl., 11:1 (2008), 43-74.

[19] И. М. Глазман, Прямые методы качественного спектрального анализа сингулярных дифференциальных операторов, Физматгиз, М., 1963, 339 с.; англ. пер.: I. M. Glazman, Direct methods of qualitative spectral analysis of singular differential operators, Izrael Program for Scientific Translations, Jerusalem, 1965; Daniel Davey \& Co., Inc., New York, 1966, ix+234 pp.

[20] A. Gogatishvili, M. Johansson, C. A. Okpoti, L.-E. Persson, "Characterisation of embeddings in Lorentz spaces", Bull. Austral. Math. Soc., 76:1 (2007), 69-92.

[21] A. Gogatishvili, A. Kufner, L.-E. Persson, A. Wedestig, "An equivalence theorem for integral conditions related to Hardy's inequality", Real Anal. Exchange, 29:2 (2003/04), 867-880.

[22] A. Gogatishvili, K. Kuliev, G. Kulieva, "Some conditions characterizing the 'reverse' Hardy inequality", Real Anal. Exchange, 33:1 (2008), 249-258.

[23] A. Gogatishvili, B. Opic, L. Pick, "Weighted inequalities for Hardy-type operators involving suprema", Collect. Math., 57:3 (2006), 227-255.

[24] A. Gogatishvili, L. Pick, "Duality principles and reduction theorems", Math. Inequal. Appl., 3:4 (2000), 539-558.

[25] A. Gogatishvili, L. Pick, "Discretization and anti-discretization of rearrangementinvariant norms", Publ. Mat., 47:2 (2003), 311-358.

[26] A. Gogatishvili, L. Pick, "Embeddings and duality theorem for weak classical Lorentz spaces", Canad. Math. Bull., 49:1 (2006), 82-95.

[27] A. Gogatishvili, L. Pick, "A reduction theorem for supremum operators", J. Comput. Appl. Math., 208:1 (2007), 270-279.

[28] А. Гогатишвили, В. Д. Степанов, "Об операторах на конусах монотонных функций”, Докл. РАН, 445:6 (2012), 618-621; англ. пер.: A. Gogatishvili, V. D. Stepanov, "Operators on cones of monotone functions", Dokl. Math., 86:1 (2012), 562-565.

[29] А. Гогатишвили, В. Д. Степанов, "Об интегральных операторах на конусах монотонных функций", Докл. РАН, 446:4 (2012), 367-370; англ. пер.: A. Gogatishvili, V.D. Stepanov, "Integral operators on cones of monotone functions", Dokl. Math., 86:2 (2012), 650-653.

[30] A. Gogatishvili, V.D. Stepanov, "Reduction theorems for operators on the cones of monotone functions", J. Math. Anal. Appl., 405:1 (2013), 156-172.

[31] М. Л. Гольдман, "Точные оценки норм операторов типа Харди на конусах квазимонотонных функций”, Функииональные пространства, гармонический анализ, дифференциальные уравнения, Сборник статей. К 95-летию со дня рождения академика Сергея Михайловича Никольского, Тр. МИАН, 232, Наука, М., 2001, 115-143; англ. пер.: M. L. Gol'dman, "Sharp estimates for the norms of Hardy-type operators on the cones of quasimonotone functions", Proc. Steklov Inst. Math., 232 (2001), 109-137.

[32] M. L. Goldman, "On equivalent criteria for the boundedness of Hardy type operators on the cone of decreasing functions", Anal. Math., 37:2 (2011), 83-102.

[33] M. L. Goldman, "Order-sharp estimates for Hardy-type operators on cones of quasimonotone functions", Eurasian Math. J., 2:3 (2011), 143-146.

[34] M.L. Goldman, "Order-sharp estimates for Hardy-type operators on the cones of functions with properties of monotonicity", Eurasian Math. J., 3:2 (2011), 53-84.

[35] M. L. Gol'dman, H. P. Heinig, V. D. Stepanov, "On the principle of duality in Lorentz spaces", Canad. J. Math., 48:5 (1996), 959-979. 
[36] М. Л. Гольдман, М. В. Сорокина, “Трехвесовые неравенства типа Харди на конусе квазимонотонных функций”, Докл. РАН, 401:3 (2005), 301-305; англ. пер.: M. L. Gol'dman, M. V. Sorokina, "Three-weighted Hardy-type inequalities on the cone of quasimonotone functions", Dokl. Math., 71:2 (2005), 209-213.

[37] М.Л. Гольдман, В.Д. Степанов, Г.П. Хайниг, "О принципе двойственности в пространствах Лоренца", Докл. РАН, 344:6 (1995), 740-744; англ. пер.: M. L. Gol'dman, V. D. Stepanov, H. P. Heinig, "On the principle of duality in Lorentz spaces", Dokl. Math., 52:2 (1995), 264-267.

[38] П. Халмош, В. Сандер, Ограниченные интегральные операторы в пространcmвах $L^{2}$, Наука, M., 1985, 159 с.; пер. с англ.: P. R. Halmos, V. S. Sunder, Bounded integral operators on $L^{2}$ spaces, Ergeb. Math. Grenzgeb., 96, Springer-Verlag, Berlin-New York, 1978, xv+132 pp.

[39] I. Halperin, "Function spaces", Canad. J. Math., 5 (1953), 273-288.

[40] Г. Г. Харди, Дж. Е. Литтлвуд, Г. Полиа, Неравенства, ИЛ, М., 1948, 456 с.; пер. с англ.: G. H. Hardy, J. E. Littlewood, G. Pólya, Inequalities, Cambridge Univ. Press, 1934, xii+314 pp.

[41] H. P. Heinig, A. Kufner, "Hardy operators on monotone functions and sequences in Orlicz spaces", J. London Math. Soc. (2), 53:2 (1996), 256-270.

[42] H. P. Heinig, Q. Lai, "Weighted modular inequalities for Hardy-type operators on monotone functions", JIPAM. J. Inequal. Pure Appl. Math., 1:1 (2000), Article 10, 25 pp. (electronic).

[43] H. P. Heinig, L. Maligranda, "Weighted inequalities for monotone and concave functions", Studia Math., 116:2 (1995), 133-165.

[44] H. P. Heinig, V. D. Stepanov, "Weighted Hardy inequalities for increasing functions", Canad. J. Math., 45:1 (1993), 104-116.

[45] M. Johansson, V. D. Stepanov, E. P. Ushakova, "Hardy inequality with three measures on monotone functions", Math. Inequal. Appl., 11:3 (2008), 393-413.

[46] W. B. Jurkat, G. Sampson, "The complete solution to the $\left(L^{p}, L^{q}\right)$ mapping problem for a class of oscillating kernels", Indiana Univ. Math. J., 30:3 (1981), 403-413.

[47] A. Kamińska, M. Mastyło, "Abstract duality Sawyer formula and its applications", Monatsh. Math., 151:3 (2007), 223-245.

[48] Л.В. Канторович, Г.П. Акилов, Функционалъный анализ, 3-е изд., Наука, М., 1984, 752 с.; англ. пер. 2-го изд.: L. V. Kantorovich, G.P. Akilov, Functional analysis, Pergamon Press, Oxford-Elmsford, NY, 1982, xiv+589 pp.

[49] В. Б. Коротков, Интегральные операторы, Наука, Новосибирск, 1983, 224 с.

[50] М.А. Красносельский, П. П. Забрейко, Е. И. Пустыльник, П.Е. Соболевский, Интегральные операторы в пространствах суммируемых функций, Наука, М., 1966, 499 с.; англ. пер.: M. A. Krasnosel'skॅ̆, P. P. Zabreı̌ko, E. I. Pustyl'nik, P. E. Sobolevskiǔ, Integral operators in spaces of summable functions, Monographs and Textbooks on Mechanics of Solids and Fluids, Mechanics: Analysis, Noordhoff International Publishing, Leiden, 1976, xv+520 pp.

[51] A. Kufner, L. Maligranda, L.-E. Persson, The Hardy inequality: About its history and some related results, Vydavatelský Servis, Plzeň, 2007, 162 pp.

[52] A. Kufner, L.-E. Persson, Weighted inequalities of Hardy type, World Scientific Publishing Co., Inc., River Edge, NJ, 2003, xviii+357 pp.

[53] Sh. Lai, "Weighted norm inequalities for general operators on monotone functions", Trans. Amer. Math. Soc., 340:2 (1993), 811-836.

[54] Q. Lai, "Weighted modular inequalities for Hardy-type operators", Proc. London Math. Soc. (3), 79:3 (1999), 649-672.

[55] E. Liflyand, S. Tikhonov, "A concept of general monotonicity and applications", Math. Nachr., 284:8-9 (2011), 1083-1098. 
[56] M. A. Lifshits, W. Linde, Approximation and entropy numbers of Volterra operators with application to Brownian motion, Mem. Amer. Math. Soc., 157, № 745, 2002, viii+87 pp.

[57] E. N. Lomakina, V.D. Stepanov, "On the compactness and approximation numbers of Hardy-type integral operators in Lorentz spaces", J. London Math. Soc. (2), 53:2 (1996), 369-383.

[58] E. N. Lomakina, V.D. Stepanov, "On the Hardy-type integral operators in Banach function spaces", Publ. Mat., 42:1 (1998), 165-194.

[59] E. N. Lomakina, V.D. Stepanov, "On asymptotic behaviour of the approximation numbers and estimates of Schatten-von Neumann norms of the Hardy-type integral operators", Function spaces and applications (Delhi, 1997), Narosa, New Delhi, 2000, 153-187.

[60] Е. Н. Ломакина, В. Д. Степанов, “Асимптотические оценки аппроксимативных и энтропийных чисел одновесового оператора Римана-Лиувилля", Матем. mp., 9:1 (2006), 52-100; англ. пер: E. N. Lomakina, V. D. Stepanov, "Asymptotic estimates for the approximation and entropy numbers of a one-weight Riemann-Liouville operator", Siberian Adv. Math., 17:1 (2007), 1-36.

[61] M. Lorente, "A characterization of two weight norm inequalities for one-sided operators of fractional type", Canad. J. Math., 49:5 (1997), 1010-1033.

[62] L. Maligranda, "Weighted inequalities for quasi-monotone functions", J. London Math. Soc. (2), 57:2 (1998), 363-370.

[63] P. J. Martin-Reyes, E. T. Sawyer, "Weighted inequalities for Riemann-Liouville fractional integrals of order one and greater", Proc. Amer. Math. Soc., 106:3 (1989), $727-733$.

[64] В.Г. Мазья, Пространства С.Л. Соболева, Изд-во Ленингр. ун-та, Л., 1985, 416 с.; англ. пер.: V. G. Mazya, Sobolev spaces, Springer Ser. Soviet Math., Springer-Verlag, Berlin, 1985, xix+486 pp.

[65] A. Meskhi, "Solution of some weight problems for the Riemann-Liouville and Weyl operators", Georgian Math. J., 5:6 (1998), 565-574.

[66] E. A. Myasnikov, L.-E. Persson, V.D. Stepanov, "On the best constants in certain integral inequalities for monotone functions", Acta Sci. Math. (Szeged), 59:3-4 (1994), 613-624.

[67] C. J. Neugebauer, "Weighted norm inequalities for averaging operators of monotone functions", Publ. Mat., 35:2 (1991), 429-447.

[68] C. J. Neugebauer, "Some classical operators on Lorentz space", Forum Math., 4:2 (1992), 135-146.

[69] J. Newman, M. Solomyak, "Two-sided estimates on singular values for a class of integral operators on the semi-axis", Integral Equations Operator Theory, 20:3 (1994), 335-349.

[70] E. Nursultanov, S. Tikhonov, "Convolution inequalities in Lorentz spaces", J. Fourier Anal. Appl., 17:3 (2011), 486-505.

[71] Р. Ойнаров, “Двусторонние оценки нормы некоторых классов интегральных операторов”, Исследования по теории дифферениируемъх функций многих переменных и ее приложениям. Часть 16, Тр. МИАН, 204, Наука, М., 1993, 240-250; англ. пер.: R. Oı̆narov, "Two-sided estimates for the norm of some classes of integral operators", Proc. Steklov Inst. Math., 204:3 (1994), 205-214.

[72] G. O. Okikiolu, Aspects of the theory of bounded integral operators in $L^{p}$ spaces, Academic Press, London-New York, 1971, ix+522 pp.

[73] L.-E. Persson, O. V. Popova, V. D. Stepanov, "Two-sided Hardy-type inequalities for monotone functions", Complex Var. Elliptic Equ., 55:8-10 (2010), 973-989.

[74] L.-E. Persson, D. Prokhorov, "Integral inequalities for some weighted geometric mean operators with variable limits", Arch. Ineq. Appl., 2:4 (2004), 475-482. 
[75] L.-E. Persson, V.D. Stepanov, "Weighted integral inequalities with the geometric mean operator", J. Inequal. Appl., 7:5 (2002), 727-746.

[76] L. E. Persson, V.D. Stepanov, E. P. Ushakova, "Equivalence of Hardy-type inequalities with general measures on the cones of non-negative respective non-increasing functions", Proc. Amer. Math. Soc., 134:8 (2006), 2363-2372.

[77] L.-E. Persson, V. D. Stepanov, P. Wall, "Some scales of equivalent weight characterizations of Hardy's inequality: the case $q<p "$, Math. Inequal. Appl., 10:2 (2007), 267-279.

[78] О. В. Попова, "Неравенства типа Харди на конусах монотонных функций”, Сиб. матем. журн., 53:1 (2012), 187-204; англ. пер.: О. V. Popova, "Hardy-type inequalities on the cones of monotone functions", Sib. Math. J., 53:1 (2012), 152-167.

[79] D. V. Prokhorov, "On the boundedness and compactness of a class of integral operators", J. London Math. Soc. (2), 61:2 (2000), 617-628.

[80] D. V. Prokhorov, "Weighted Hardy's inequalities for negative indices", Publ. Mat., 48:2 (2004), 423-443.

[81] Д.В. Прохоров, "Неравенство Харди с тремя мерами", Функииональные пространства, теория приближений, нелинейный анализ, Сборник статей, Тр. МИАН, 255, Наука, М., 2006, 233-245; англ. пер.: D. V. Prokhorov, "Hardy's inequality with three measures", Proc. Steklov Inst. Math., 255:1 (2006), 221-233.

[82] D. V. Prokhorov, "Inequalities of Hardy type for a class of integral operators with measures", Anal. Math., 33:3 (2007), 199-225.

[83] D. V. Prokhorov, "Inequalities for Riemann-Liouville operator involving suprema", Collect. Math., 61:3 (2010), 263-276.

[84] D. V. Prokhorov, "Lorentz norm inequalities for the Hardy operator involving suprema", Proc. Amer. Math. Soc., 140:5 (2012), 1585-1592.

[85] D. V. Prokhorov, A new characterization of the Hardy-type weighted norm inequalities, Res. Rep. 2013/192, Computing Centre FEB RAS, Khabarovsk, 2013, 11 pp.

[86] Д. В. Прохоров, В. Д. Степанов, "Весовые оценки операторов Римана-Лиувилля и приложения", Функциональные пространства, приближения, дифференциальные уравнения, Сборник статей. К 70-летию со дня рождения члена-корреспондента РАН Олега Владимировича Бесова, Тр. МИАН, 243, Наука, М., 2003, 289-312; англ. пер.: D. V. Prokhorov, V.D. Stepanov, "Weighted estimates of Riemann-Liouville operators and applications", Proc. Steklov Inst. Math., 243:4 (2003), 278-301.

[87] E. Sawyer, "Boundedness of classical operators on classical Lorentz spaces", Studia Math., 96:2 (1990), 145-158.

[88] G. Sinnamon, "Spaces defined by the level functions and their duals", Studia Math., 111:1 (1994), 19-52.

[89] G. Sinnamon, "Embeddings of concave functions and duals of Lorentz spaces", Publ. Mat., 46:2 (2002), 489-515.

[90] G. Sinnamon, "Transferring monotonicity in weighted norm inequalities", Collect. Math., 54:2 (2003), 181-216.

[91] G. Sinnamon, "Hardy's inequality and monotonicity", Function spaces, differential operators and nonlinear analysis, Proc. Conf. (Milovy, Czech Republ., May 28-June 2, 2004), eds. P. Drábec, J. Rákosnik, Math. Inst. Acad. Sci. Czech Republ., Prague, $2005,292-310$.

[92] G. Sinnamon, V.D. Stepanov, "The weighted Hardy inequality: new proofs and the case $p=1 "$, J. London Math. Soc. (2), 54:1 (1996), 89-101.

[93] M. Solomyak, "Estimates for the approximation numbers of the weighted Riemann-Liouville operator in the spaces $L_{p}{ }^{\prime}$, Complex analysis, operators, and related topics, Oper. Theory Adv. Appl., 113, Birkhäuser, Basel, 2000, 371-383. 
[94] J. Soria, "Lorentz spaces of weak-type", Quart. J. Math. Oxford Ser. (2), 49:1 (1998), 93-103.

[95] V.D. Stepanov, Two-weighted estimates for Riemann-Liouville integrals, Rep. № 39, Math. Inst. Czechoslovak Acad. Sci., Prague, 1988, 28 pp.

[96] В.Д. Степанов, “Об одном весовом неравенстве типа Харди для производных высших порядков", Исследования по теории дифференцируемых функиий многих переменных и ее приложениям. Часть 13, Сборник работ, Тр. МИАН СССР, 187, Наука, M., 1989, 178-190; англ. пер.: V. D. Stepanov, "On a weighted inequality of Hardy type for derivatives of higher order", Proc. Steklov Inst. Math., 187:3 (1990), 205-220.

[97] В.Д. Степанов, “О весовых неравенствах типа Харди для дробных интегралов Римана-Лиувилля", Сиб. матем. журн., 31:3 (1990), 186-197; англ. пер.: V.D. Stepanov, "Weight inequalities of Hardy type for fractional Riemann-Liouville integrals", Sib. Math. J., 31:3 (1990), 513-522.

[98] В. Д. Степанов, “Двухвесовые оценки для интегралов Римана-Лиувилля", Изв. AH CCCP. Сер. матем., 54:3 (1990), 645-656; англ. пер.: V.D. Stepanov, "Two-weighted estimates of Riemann-Liouville integrals", Math. USSR-Izv., 36:3 (1991), 669-681.

[99] В. Д. Степанов, "Об ограниченности линейных интегральных операторов на классе монотонных функций”, Сиб. матем. журн., 32:3 (1991), 222-224; англ. пер.: V.D. Stepanov, "Boundedness of linear integral operators on a class of monotone functions", Sib. Math. J., 32:3 (1991), 540-542.

[100] V.D. Stepanov, "Weighted inequalities for a class of Volterra convolution operators", J. London Math. Soc. (2), 45:2 (1992), 232-242.

[101] В.Д. Степанов, Д.Э. Эдмундс, “О мере некомпактности и аппроксимативных числах одного класса вольтерровских интегральных операторов", Докл. PAH, 330:6 (1993), 700-703; англ. пер.: V. D. Stepanov, D. E. Edmunds, "On the measure of noncompactness and approximation numbers of a class of Volterra integral operators", Russian Acad. Sci. Dokl. Math., 47:3 (1993), 618-623.

[102] В. Д. Степанов, "О весовых оценках одного класса интегральных операторов", Сиб. матем. журн., 34:4 (1993), 184-196; англ. пер.: V. D. Stepanov, "On weighted estimates for a class of integral operators", Sib. Math. J., 34:4 (1993), 755-766.

[103] V.D. Stepanov, "The weighted Hardy's inequality for nonincreasing functions", Trans. Amer. Math. Soc., 338:1 (1993), 173-186.

[104] V.D. Stepanov, "Integral operators on the cone of monotone functions", J. London Math. Soc. (2), 48:3 (1993), 465-487.

[105] В. Д. Степанов, "О сингулярных числах одного класса интегральных операторов", Докл. РАН, 336:4 (1994), 457-458; англ. пер.: V.D. Stepanov, "On the singular numbers of a class of integral operators", Russian Acad. Sci. Dokl. Math., 49:3 (1994), 545-547.

[106] V.D. Stepanov, "Weighted norm inequalities of Hardy type for a class of integral operators", J. London Math. Soc. (2), 50:1 (1994), 105-120.

[107] V. D. Stepanov, "Weighted norm inequalities for integral operators and related topics", Nonlinear analysis, function spaces and applications, v. 5, Proceedings of Spring School (Prague, 1994), Prometheus, Prague, 1994, 139-175.

[108] В. Д. Степанов, Некоторые вопросы теории интегральных операторов свертки, Дальнаука, Владивосток, 2000, 228 с.

[109] V. D. Stepanov, "On the lower bounds for Schatten-von Neumann norms of certain Volterra integral operators", J. London Math. Soc. (2), 61:3 (2000), 905-922.

[110] V.D. Stepanov, "On a supremum operator", Spectral theory, function spaces and inequalities, Oper. Theory Adv. Appl., 219, Birkhäuser/Springer Basel AG, Basel, 2012, 233-242. 
[111] В.Д. Степанов, С. Ю. Тихонов, “Двухвесовые неравенства для преобразования Гильберта на монотонных функциях", Докл. РАН, 437:5 (2011), 606-608; англ. пер.: V. D. Stepanov, S. Yu. Tikhonov, "Two-weight inequalities for the Hilbert transform on monotone functions", Dokl. Math., 83:2 (2011), 241-242.

[112] V.D. Stepanov, S. Yu. Tikhonov, "Two power-weight inequalities for the Hilbert transform on the cones of monotone functions", Complex Var. Elliptic Equ., 56:10-11 (2011), 1039-1047.

[113] В.Д. Степанов, Е.П. Ушакова, "Весовые оценки для интегральных операторов на полуоси с монотонными ядрами", Сиб. матем. журн., 45:6 (2004), 1378-1390; англ. пер.: V.D. Stepanov, E. P. Ushakova, "Weighted estimates for the integral operators with monotone kernels on a halh-axis", Sib. Math. J., 45:6 (2004), 1124-1134.

[114] В. Д. Степанов, Е.П. Ушакова, “Об операторе геометрического среднего с переменными пределами интегрирования”, Теория функций и нелинейные уравнения в частных производных, Сборник статей. К 70-летию со дня рождения члена-корреспондента РАН Станислава Ивановича Похожаева, Тр. МИАН, 260, МАИК, М., 2008, 264-288; англ. пер.: V.D. Stepanov, E.P. Ushakova, "On the geometric mean operator with variable limits of integration", Proc. Steklov Inst. Math., 260:1 (2008), 254-278.

[115] V.D. Stepanov, E. P. Ushakova, "Alternative criteria for the boundedness of Volterra integral operators in Lebesgue spaces", Math. Inequal. Appl., 12:4 (2009), 873-889.

[116] V. D. Stepanov, E. P. Ushakova, "Kernel operators with variable intervals of integration in Lebesgue spaces and applications", Math. Inequal. Appl., 13:3 (2010), 449-510.

[117] J. Q. Sun, "The modular inequalities for a class of convolution operators on monotone functions", Proc. Amer. Math. Soc., 125:8 (1997), 2293-2305.

[118] А. А. Васильева, "Оценки поперечников весовых соболевских классов", Матем. сб., 201:7 (2010), 15-52; англ. пер.: А. A. Vasil'eva, "Estimates for the widths of weighted Sobolev classes", Sb. Math., 201:7 (2010), 947-984.

A. Гогатишвили (A. Gogatishvili)

Mathematical Institute, Academy of Sciences

of the Czech Republic

E-mail: gogatish@math.caz.cz

В. Д. Степанов (V.D. Stepanov)

Российский университет дружбы народов

E-mail: stepanov@mi.ras.ru
Поступила в редакцию 02.02.2013 